University of Louisville

ThinkIR: The University of Louisville's Institutional Repository

Electronic Theses and Dissertations

$5-2018$

\title{
Challenges in flexible microsystem manufacturing : fabrication, robotic assembly, control, and packaging.
}

Danming Wei

University of Louisville

Follow this and additional works at: https://ir.library.louisville.edu/etd

Part of the Controls and Control Theory Commons, Electrical and Electronics Commons, Electronic Devices and Semiconductor Manufacturing Commons, and the Nanotechnology Fabrication Commons

\section{Recommended Citation}

Wei, Danming, "Challenges in flexible microsystem manufacturing : fabrication, robotic assembly, control, and packaging." (2018). Electronic Theses and Dissertations. Paper 2921.

https://doi.org/10.18297/etd/2921

This Master's Thesis is brought to you for free and open access by ThinkIR: The University of Louisville's Institutional Repository. It has been accepted for inclusion in Electronic Theses and Dissertations by an authorized administrator of ThinkIR: The University of Louisville's Institutional Repository. This title appears here courtesy of the author, who has retained all other copyrights. For more information, please contact thinkir@louisville.edu. 
CHALLENGES IN FLEXIBLE MICROSYSTEM MANUFACTURING: FABRICATION, ROBOTIC ASSEMBLY, CONTROL, AND PACKAGING

\author{
By \\ Danming Wei \\ A Thesis \\ submitted to the Faculty of the \\ J. B. Speed School of Engineering of the University of Louisville \\ in Partial Fulfilment of the Requirements \\ For the Degree of \\ Master of Science in Electrical Engineering \\ Department of Electrical and Computer Engineering \\ University of Louisville \\ Louisville, Kentucky
}

May 2018 
Copyright 2018 by Danming Wei

All rights reserved 

CHALLENGES IN FLEXIBLE MICROSYSTEM MANUFACTURING: FABRICATION, ROBOTIC ASSEMBLY, CONTROL, AND PACKAGING

By

Danming Wei

A Thesis Approved on

April 20, 2018

by the following Thesis Committee:

Dr. Dan O. Popa

Dr. Shamus McNamara

Dr. Cindy Harnett 


\section{DEDICATION}

This thesis is dedicated to my parents

Mr. Chang Wei

And

Mrs. Lizhi Guo

Who have given me invaluable educational opportunities 


\section{ACKNOWLEDGMENTS}

I would first like to thank my supervising professor Dr. Dan O. Popa for his extensive guidance and continuous support for my master's degree study and related research. In addition, I would also like to thank Dr. Shamus McNamara and Dr. Cindy Harnett for serving on my thesis committee.

I am thankful to the Department of Electrical and Computer Engineering at the University of Louisville for providing me the opportunity to serve as a graduate teaching assistant, which improved my communication skills and enhanced my confidence. I am grateful to the Next Generation Systems (NGS) research group for all the support and friendship that they have given. Moreover, I owe thanks to Dr. Popa for giving me the chance to participate in Mobile Microrobot Challenge 2017 in Singapore, which provided me valuable experience in international conferences and events.

I truly appreciate the support from my parents and girlfriend who have encouraged me to move forward to ultimate success. This thesis is dedicated to them with whole-hearted love and gratitude. 


\begin{abstract}
CHALLENGES IN FLEXIBLE MICROSYSTEM MANUFACTURING: FABRICATION, ROBOTIC ASSEMBLY, CONTROL, AND PACKAGING

Danming Wei
\end{abstract}

April 27, 2018

Microsystems have been investigated with renewed interest for the last three decades because of the emerging development of microelectromechanical system (MEMS) technology and the advancement of nanotechnology. The applications of microrobots and distributed sensors have the potential to revolutionize micro and nano manufacturing and have other important health applications for drug delivery and minimal invasive surgery. A class of microrobots studied in this thesis, such as the Solid Articulated Four Axis Microrobot (sAFAM) are driven by MEMS actuators, transmissions, and end-effectors realized by 3-Dimensional MEMS assembly. Another class of microrobots studied here, like those competing in the annual IEEE Mobile Microrobot Challenge event (MMC) are untethered and driven by external fields, such as magnetic fields generated by a focused permanent magnet. A third class of microsystems studied in this thesis includes distributed MEMS pressure sensors for robotic skin applications that are manufactured in the cleanroom and packaged in our lab. 
In this thesis, we discuss typical challenges associated with the fabrication, robotic assembly and packaging of these microsystems. For sAFAM we discuss challenges arising from pick and place manipulation under microscopic closed-loop control, as well as bonding and attachment of silicon MEMS microparts. For MMC, we discuss challenges arising from cooperative manipulation of microparts that advance the capabilities of magnetic micro-agents. Custom microrobotic hardware configured and demonstrated during this research (such as the NeXus microassembly station) include micro-positioners, microscopes, and controllers driven via LabVIEW. Finally, we also discuss challenges arising in distributed sensor manufacturing. We describe sensor fabrication steps using clean-room techniques on Kapton flexible substrates, and present results of lamination, interconnection and testing of such sensors are presented. 


\section{TABLE OF CONTENTS}

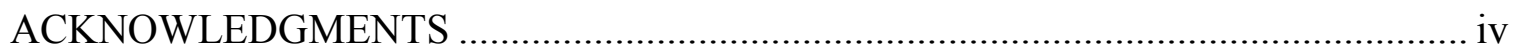

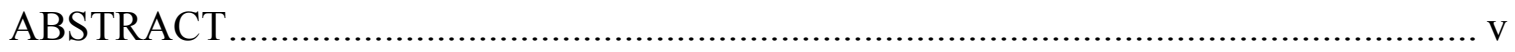

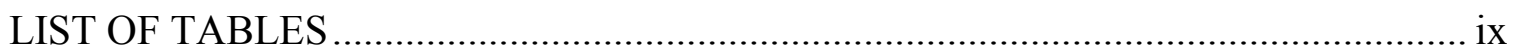

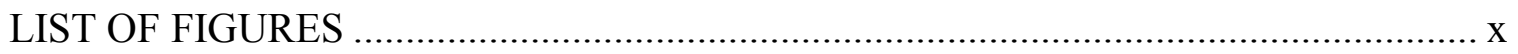

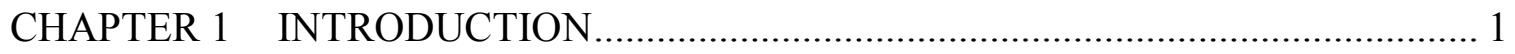

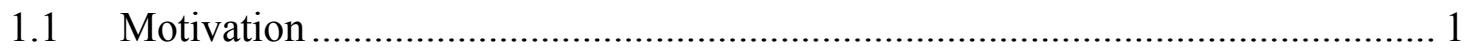

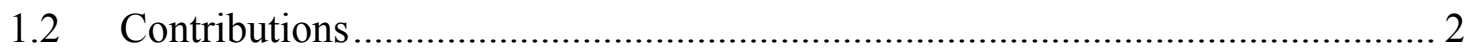

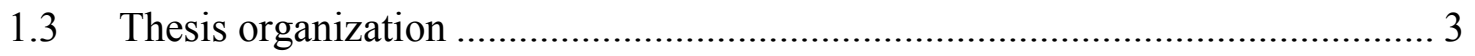

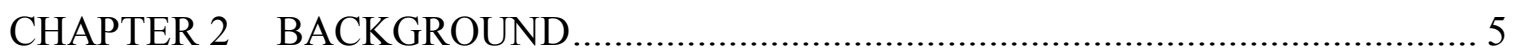

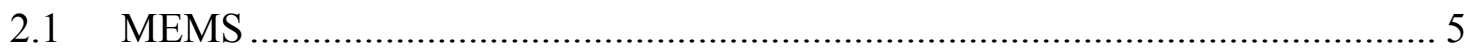

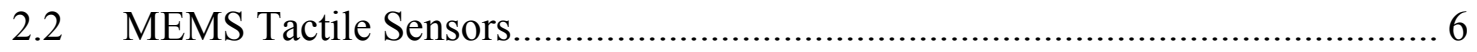

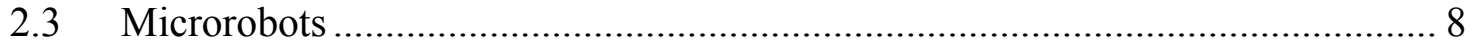

2.3.1 Field actuated microrobots................................................................. 9

2.3.2 Biomimetic non-magnetic microrobots ................................................. 10

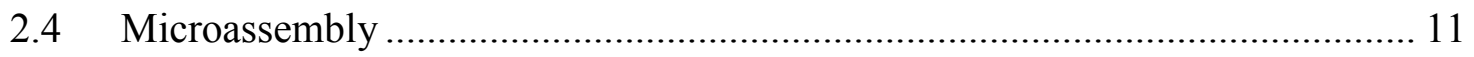

CHAPTER 3 MICROROBOTS …………………………................................. 13

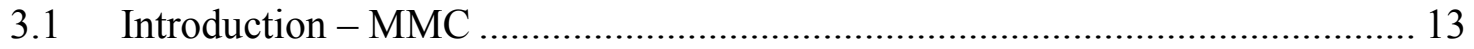

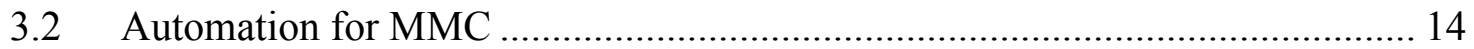

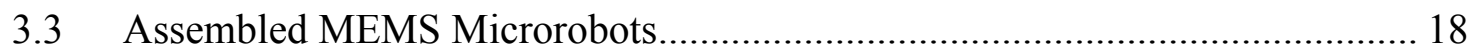

CHAPTER 4 NEXUS MICROASSEMBLY SYSTEM DESIGN................................. 22

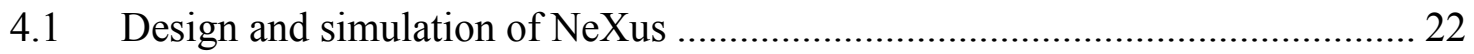

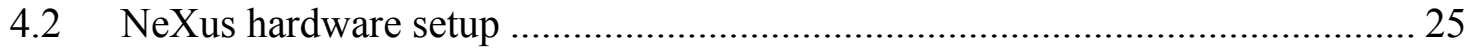

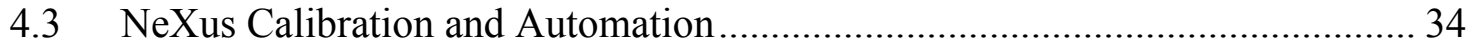

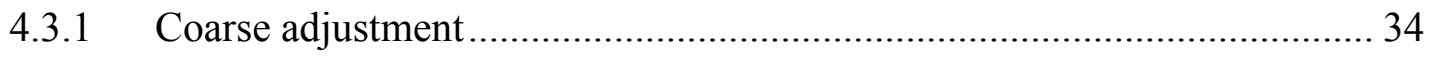

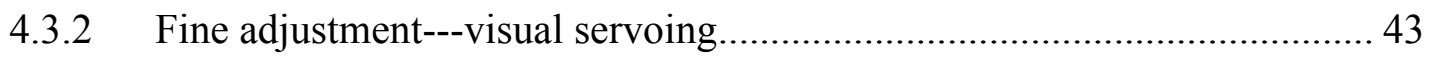

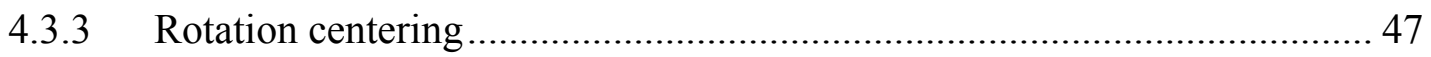

4.3.4 3-point teaching method …………………….................................... 48 


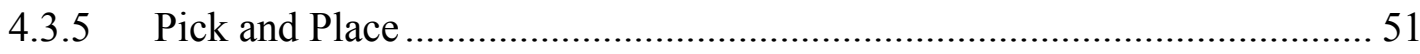

4.3.5.1 Jammer part assembly testing .................................................... 51

4.3.5.2 sAFAM assembly and bonding ..................................................... 52

CHAPTER 5 FLEXIBLE SKIN SENSOR FABRICATION AND EVALUATION ... 55

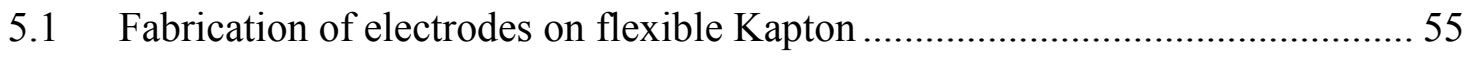

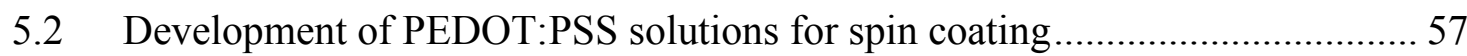

CHAPTER 6 CONCLUSION AND FUTURE WORK............................................ 63

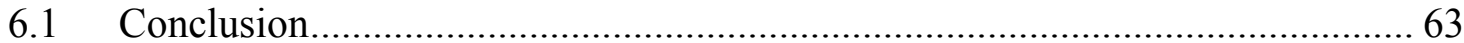

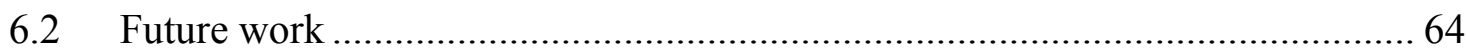

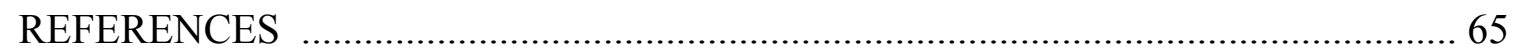

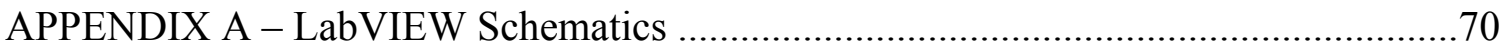

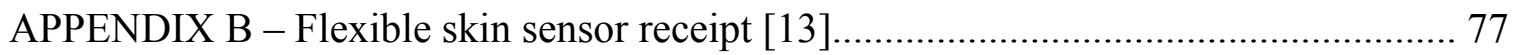

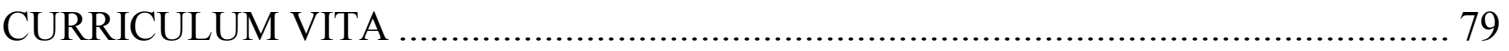




\section{LIST OF TABLES}

Table 4.1. The parameters of all motorized stages for M1 and M2 ............................. 32

Table 4.2. The new coordinates of each template center in reference to the die holder

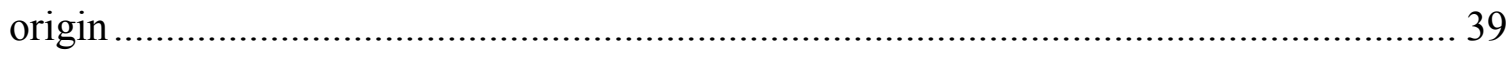

Table 4.3. 16 testing points moving to desired position with 0 -degree orientation .......... 40

Table 4.4. 16 testing points moving to desired position with 30 -degree orientation........ 41

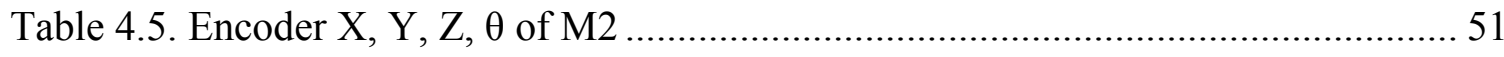

Table 4.6. Comparison of sAFAM experiment and simulation results .......................... 54

Table 5.1. Measurements of sheet resistance and resistivity for PEDOT:PSS thin films

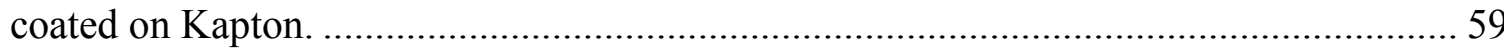

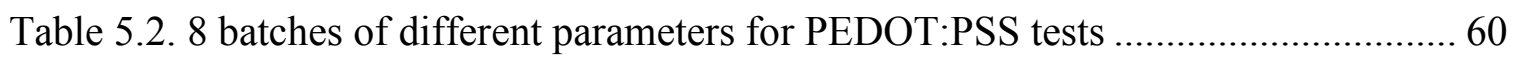




\section{LIST OF FIGURES}

Figure 3.1. MMC arena definition (a), an MMC path sample (b), and definition of hit and

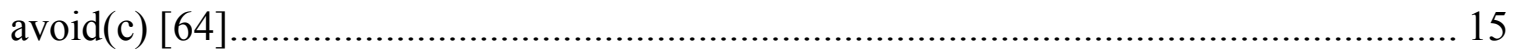

Figure 3.2. MMC2017 hardware setup and corresponding components ....................... 16

Figure 3.3. MMC2017 first challenge main interface.............................................. 16

Figure 3.4. Three tasks involved in the first challenge ........................................... 17

Figure 3.5. Area dimensions and sample of assembly for the second challenge.............. 17

Figure 3.6. MMC2017 second challenge main interface ............................................. 18

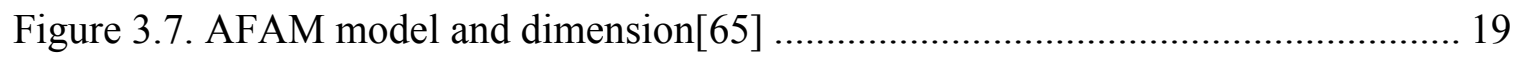

Figure 3.8. AFAM arm with Zyvex snap-fastener and socket................................... 20

Figure 3.9. 3D model of sAFAM from SolidWorks ${ }^{\mathrm{TM}}$.............................................. 21

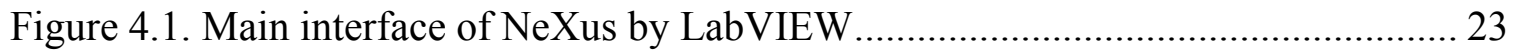

Figure 4.2. Distribution of 4 manipulations in LabVIEW simulation of NeXus............. 24

Figure 4.3. Steps necessary to split a linear stage CAD file into several movable parts .. 25

Figure 4.4. Recent physical configuration of the NeXus robotic microassembly system 26

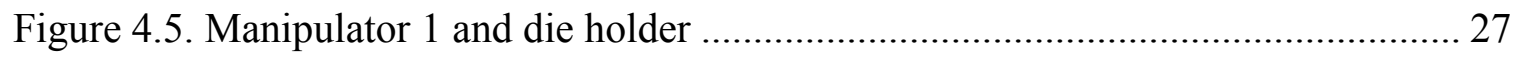

Figure 4.6. Manipulator 2 with end-effector mounted microjammer ............................ 28

Figure 4.7. Vertical imaging system with zoom lens, stepping motor control, and

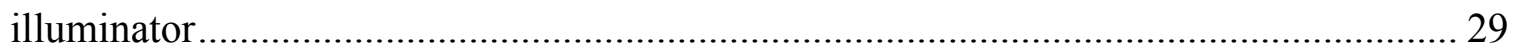

Figure 4.8. Horizontal imaging system and illuminator with gooseneck optic fiber........ 30 


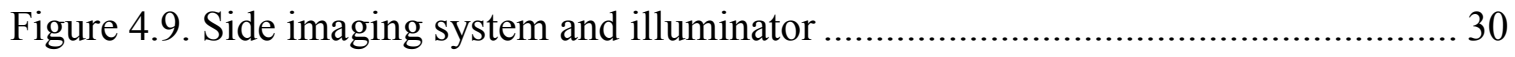

Figure 4.10. Vacuum pen for loading and unloading dies ...................................... 31

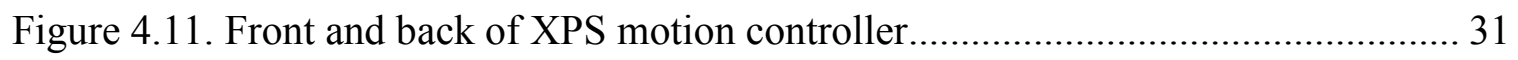

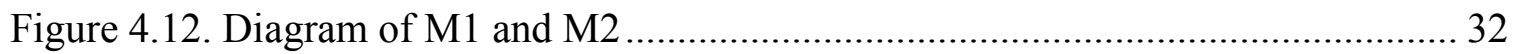

Figure 4.13. Single head and dual heads microjammers mounted on the end-effector .... 33

Figure 4.14. Side and top view of single-head microjammer mounted on the end-effector

Figure 4.15. Arbitrary point moves to desired configuration .................................... 35

Figure 4.16. Interface for M1 coarse adjustment ................................................. 36

Figure 4.17. Die distribution and coordinates of upper left corner in reference to the

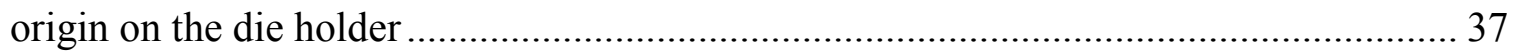

Figure 4.18. Template center coordinates in reference to the origin in the die layout

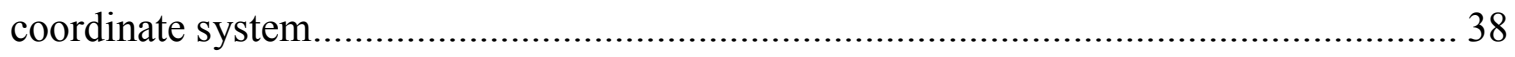

Figure 4.19. Socket template dimensions and three points coordinate in pixel............... 42

Figure 4.20. Test of desired point movement .......................................................... 43

Figure 4.21. Three interfaces for semi-automation calibration of M1 .......................... 47

Figure 4.22. Rotation centering for the microjammer tip ..................................... 48

Figure 4.23. Manual translation stage for rotation centering .................................... 48

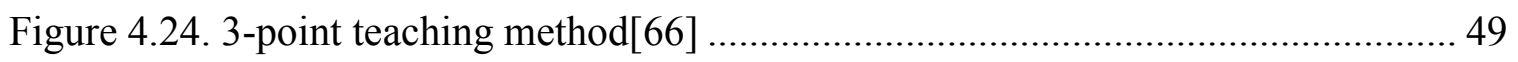

Figure 4.25. Single-head microjammer to calibrate M1 ......................................... 51

Figure 4.26. Pick-rotate-place for part assembly testing and assembled AFAM ........... 52

Figure 4.27. Picking-up, rotating, and placing for sAFAM assembly .......................... 53

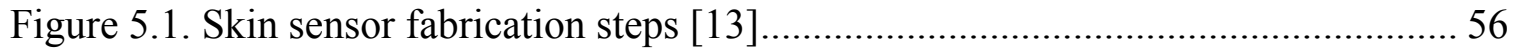


Figure 5.2. Examples of IDE structure made of gold on Kapton Sheet [13] ................... 58

Figure 5.3. 5-point measurements on Kapton sheet by 4-point probe station .................. 59

Figure 5.4. Resistances measured on skin sensor heads and tails with 8 batches of recipes

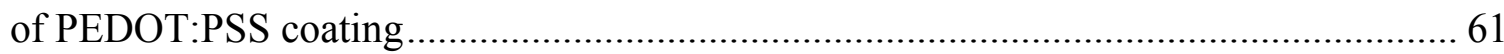

Figure 5.5. Probe station for skin sensor resistance measurement on head and tails ....... 62 


\section{CHAPTER 1}

\section{INTRODUCTION}

\subsection{Motivation}

Microrobotics technology is an emerging approach to microsystem technology and has been developing rapidly over the last several decades. The potential applications of microrobotics have extended to areas including military surveillance, microsystem and nanosystem manufacturing of tiny industrial components, and sample manipulation in biology for cellular, laboratory analysis and surgical applications [1-6]. The study of microrobots has attracted much attention due to the design and exploration of new microrobot structures and functions with the help of Micro Electro Mechanical Systems (MEMS) technology that can fabricate microstructures on a Silicon substrate.

Microassembly systems using precision robots or microrobots have been demonstrated to be vitally indispensable to micro and nano manufacturing. The resulting microassembly systems can provide flexible and functional assembly techniques for manufacturing complex microrobots, which can advance further research studies of such microrobots.

Motivated by biological structures found in nature, robotic skin is a significant type of exteroceptive sensor which can eventually lead to robots working side by side with humans. Despite considerable progress in the development of robotic skin sensors in the last 30 years, numerous fabrication, integration, dynamic performance, reliability, and cost challenges remain for fully realizing robotic skins, which is why several projects 
around the world have continued investigating this technology [7-13]. To enable the interaction between humans and robots, the robots should have sensory features (analogous to human skin) to feel the surroundings. Flexible tactile sensors distributed over the body or hands of a robot become indispensable core components to assist in the understanding of environmental surroundings and in physical communications with humans.

\subsection{Contributions}

a) During my research, I have designed, configured and controlled a new laboratory instrument: The NeXus microassembly system, which is used as a prototype platform for $2 \frac{1}{2}$ D MEMS microrobots. In order to test the functionality of NeXus, I assembled the Articulated Four Axis Microrobot (AFAM), and an updated design, the Solid AFAM (sAFAM) microrobot. These microrobots were used as test vehicles to improve and optimize the design and programming of the assembly system. During this research, several challenges related to micropart fixturing, gripping, positioning, and snap-fast assembly were overcome.

b) To further understand and develop microrobots technology with applications in manufacturing and medicine, I participated in the IEEE Mobile Microrobot (MMC) 2017 competition, which was held at the 2017 International Conference on Robotics and Automation in Singapore. I adopted a conical magnet to generate a movable, untethered magnetic field which actuated and controlled the magnetic microrobot. Control of microrobots using electromagnetic fields has been employed by many other groups in the past [14-18]. In my research, I employed a focused magnetic field to accomplish precise positioning and pushing tasks on the substrate. Challenges that 
needed to be overcome relate to the programming of automated microrobot movements, and teleoperation of microrobots to accomplish microobject pushing tasks under microscopic feedback.

c) Finally, I conducted research on the fabrication of flexible skin sensors for physical human-robot interaction applications. By using a novel wet lift-off photolithographic technique to coat a polymer piezoresistive material - the Poly(3,4-ethylene dioxythiophene)-poly(styrene sulfonate) or PEDOT: PSS -, we have prototyped distributed arrays of pressure sensors with good electrical and thermal characteristics[13].

My research resulted in the publications of two conference papers at International Conferences, including:

1. J. R. Baptist, R. Zhang, D. Wei, M. N. Saadatzi, and D. O. Popa, "Fabrication of strain gauge based sensors for tactile skins," in Smart Biomedical and Physiological Sensor Technology XIV, 2017, vol. 10216, p. 102160F: International Society for Optics and Photonics.

2. R. Zhang, D. Wei, and D. O. Popa, "Design, Analysis and Fabrication of sAFAM, a 4 DoF Assembled Microrobot," in Proceedings of IEEE International Conference on Manipulation, Automation, and Robotics at Small Scales (MARSS), 5-7 July, Nagoya, Japan, 2018 (to appear).

\subsection{Thesis organization}

In this thesis, I am reporting on some of the challenges characteristic of flexible microsystem manufacturing, particularly those in fabrication, robotic assembly, control, 
and packaging. The thesis is organized as follows: Chapter 2 includes background literature review in the areas of MEMS, microrobots, and microassembly. Chapter 3 discusses the research results in magnetic control for microrobots. Chapter 4 describes the NeXus microassembly system, and its use to prototype $2 \frac{1}{2} \mathrm{D}$ microrobots such as the AFAM. In Chapter 5, we present the fabrication and evaluation of the flexible skin sensors. Finally, Chapter 6 concludes the thesis and discusses plans for future work. 


\section{CHAPTER 2}

\section{BACKGROUND}

\subsection{MEMS}

MEMS is an acronym for Micro Electro-Mechanical Systems, whose technology is inherited from the field of integrated circuits fabrication technology. In general, MEMS refers to a series of processes, microscale structure designs, and their fabrication, leading to application of sensing and actuation interaction with local surroundings [19]. As a revolutionary enabling technology, MEMS has experienced several decades of rapid development based on its outstanding applications in various fields. Additionally, MEMS technology benefits from its tiny size, low weight, excellent performance, ease of massproduced, and relatively low cost [20]. Because of these advantages, a strong marketing push drives the development and the expandability of MEMS products into our daily life, which promises further market growth. Meanwhile, MEMS technology has promoted the development of a multitude of emerging devices, such as inkjet printers, gyroscopes, drug delivery systems, and so forth [21]. Based on MEMS components' different application areas, they can be classified as follows [19]:

- Sensors: MEMS components, which are designed to interact, generate changes with their surroundings, and provide the feedback signals for a closed-loop control system. The most common MEMS sensors include pressure, motion, optical, thermal, acceleration, inertia, and strain sensors, etc. 
- Actuators: MEMS devices are used to provide stimulus or power to other MEMS devices or components. Typical MEMS actuators are mostly driven by thermal or electrostatically methods.

- RF MEMS: radio frequency MEMS devices are employed to work a high frequency, and RF signals transmission or switch. Typical components cover metal contact switches and antennas, etc.

- Optical MEMS: they are designed as, components with optical functions such as switches and reflectors to filter or amplify and reflect light.

- Microfluidic MEMS: MEMS components are designed to work in fluidic environments. MEMS valves and pumps have been used to move, eject, and mix tiny volumes of fluid.

- Bio MEMS: similar to microfluidic MEMS, they are designed to work with biological samples, like biological cells and medical reagents, to analyze in-situ medical conditions or deliver drugs to the targets.

From the examples given in these six categories, it is obvious that current MEMS devices have different applications for use or development in commercial and government contexts.

\subsection{MEMS Tactile Sensors}

For the last several years, engineers and researchers have investigated tactile sensors dependent on MEMS technology. Robotic skin sensors are one type of tactile sensors which can be applied to "haptic interfaces, robotic manipulation, and physical human-robot interaction"[13]. 
Despite considerable progress in the last 30 years with this "holy grail" product, numerous challenges of fabrication, integration, dynamic performance, reliability, and cost challenges remain in realizing robotic skins. This is why several projects around the world have continued investigating this technology [7-12].

In the last few years, members of the Next Generation Systems Lab have made advances in fabricating, packaging, and interconnecting tactile-sensitive skins to robots, and several papers have been published at past SPIE events [22-25]. In past work, sensor geometries representing well known Interdigitated Element (IDE) structures were patterned onto flexible Kapton ${ }^{\circledR}$ substrates, and a polymer piezo-resistive material, Poly (3,4-ethylene dioxythiophene)-poly (styrene sulfonate) or PEDOT: PSS, was deposited onto sensor sites using Electro Hydro Dynamic (EHD) printing.

Thus, sensor skins consist of arrays of strain gauges with a high gauge factor (GF) [26], that can relate applied pressure to strain based on the well-known and characterized performance of the electrical properties of PEDOT: PSS on IDE structures [27-32]. EHD printing improves upon conventional ink-jet printing of PEDOT: PSS [33] by delivering on-demand jetting of nanometric film thicknesses. However, EHD inks needed special formulation due to conductivity and viscosity requirements of the process. Furthermore, the serial nature of the printing process makes manufacturing of high density skins difficult.

As a result, our research investigates the feasibility of patterning PEDOT: PSS using photolithographic methods that can be batch fabricated using standard clean-room equipment. Photolithographic methods for patterning PEDOT: PSS have been 
investigated by many others in the context of Organic LEDs, polymer transistors and other electronic display technologies [34-40].

\subsection{Microrobots}

Based on the precise motion by different microstructures as the actuators driven with internal or external force or energy on MEMS technology, microrobots' applications and developments have been increasingly attractive for medical applications, especially for diagnosis and surgery. A variety of micro actuators have been actively investigated for their potential applications, such as electrostatic, piezoelectric (PZT), giant magnetostrictive (GMA), shape memory alloys (SMA), polymer actuators, and optical tweeze actuators [41]. Microrobots in biomedical applications are driven inside blood vessels for minimally invasive medicine; therefore, microsurgery is a fertile field for biomimetic microrobot designs that operate in in-pipe mechanisms. Microrobots with actuating and sensing elements, which are able to swim smoothly in liquid media, are investigated for microsurgery of blood vessels and pipe inspection [42].

How to drive microrobots with controllable and precise motion becomes the focused topic in all applications. Main methodologies that drive microrobots are mechanical and physical properties of material applied to microrobots. For example, untethered magnetic or electromagnetic fields, which are generated by permanent magnets or electromagnetic coils respectively, can drive the microrobots with specific

material coatings or with their inherent physical magnetic properties, that are reactive in a magnetic field. Meanwhile, the microrobots with mechanical microstructure actuators can be driven by other wireless external energy, like laser, solar, vibration, and so on. In 
general, there are two main categories for classifying microrobots: field actuated microrobots and biomimetic microrobots as described below.

\subsubsection{Field actuated microrobots}

Currently, these types of microrobots are investigated mainly for medical applications. One of their features is that such microrobots can work in a very tiny spaces and primarily in fluidic environments. Pan et al. and Zhang et al $[42,43]$ have conducted research on fish-like underwater microrobots with new models driven by an external magnetic field. Abbott et al [44] discussed how biomimetic microrobots can swim via an external untethered magnetic field. Zhang [45] presented a 30-micron-long artificial bacterial flagella microrobot which rotated in a magnetic field.

Using external magnetic energy fields to actuate microrobots is a central approach in the work of many other research groups. Abbott et al [44] and Floyd et al [18, 46, 47] have investigated untethered magnetic field by using electromagnets to control the microrobots. They used the Helmholtz coils to build up the hardware which can generate an untethered magnetic field. Kummer et al [3] built up "OctoMag", which is an electromagnetic wireless micromanipulation system with 5 degrees of freedom. The OctoMag is composed of eight electromagnet coils, which are cylinders $210 \mathrm{~mm}$ long and $62 \mathrm{~mm}$ in diameter, which control a fully untethered microrobot with 5 degrees of freedom (DOF) including 3 DOFs for positions and 2 DOFs for pointing orientations.

The microrobots which are applied in a magnetic field are fabricated using two main approaches. One type of material is ferromagnetic, such as Nickel, which becomes magnetized in the presence of the magnetic field. Another type of material is a permanent 
magnet that can be assembled into the passively flexible body of the robot. Yesin et al [48] assembled microrobots with Nickel components and deployed them in a fluidic environment by utilizing electromagnetic fields. Sudo et al [49] designed a fish-like microrobot with a magnetic head and elastic tail to explore the magnetic swimming mechanism in a viscous liquid. Abbott et al [44] developed a fish-like microrobot with a helical propeller tail to investigate how it works in a rotating uniform magnetic field.

\subsubsection{Biomimetic non-magnetic microrobots}

In addition to the biomimetic microrobots mentioned in the previous section, Guo [41] presented a novel type of an underwater biomimetic fish-like microrobot driven by an ionic conducting polymer film (ICPF) actuator to produce a swimming motion with three degrees of freedom. In addition, Wood et al $[50,51]$, Lok et al [52], and Ma et al [53] designed and developed the Harvard RoboBee which are robotic air vehicles on an insect-scale that utilize flapping wings flight actuated by two bimorph piezoelectric actuators. Chen et al [54] came up with the new design of flapping wings which gave the Harvard RoboBee a hybrid potential to work in aerial and aquatic environments. Biomimetic microrobots are not only swimming in liquid environment and flying in aerial environment, but some microrobots also can crawl on the surface with a variety of mechanically structured legs.

Murthy et al [55] designed an assembled die-scale microcrawler, called "ARRIpede", which is using an electronic backpack to control a multi-legged micromechanical module. The high-stiffness micromechanical legs are assembled by a microassembly system platform and driven by electrothermal actuators. The movement 
of microcrawlers is programmed by the electronic PCB on the backpack. The motion mechanism relies on a stick-slip mechanical dynamic movement.

\subsection{Microassembly}

Robotic microassembly technology has been extensively studied for last three decades. Before that, researchers relied on manual operations to assemble micro mechanical components and manipulate biological cells [56]. When higher volumes, smaller parts, and higher precision are required, or more extreme cleanliness is needed, robotic assembly lines or robot work cells beyond human tools are needed [57]. There is an increasing recognition that more complex and more useful microsystems comprised of various materials such as $\mathrm{Si}, \mathrm{GaAs}$, metals, and plastics will require some kinds of assembly [58]. In other words, without assembly, a monolithically fabricated MEMS will be limited in the number of materials it can incorporate [57]. In general, microassembly methods can be classified in two main approaches as serial and parallel.

Serial microassembly provides a traditional "pick and place" methods to assemble microparts in $2 \frac{1}{2} \mathrm{D}$ or 3 -dimensional structures. For this process, the first step is to pick up a micropart from a planar substrate using a robotic manipulator. The next step is to translate and/or rotate the micropart relative to the assembly target. The final step is to add the micropart to an intermediate position for further operation or to its desired position [59]. The robotic manipulators used for serial microassembly processes are composed of a precision positioner terminated by a microgripper, microtweezer, and/or a microjammer, which are selected based on different micropart geometry. During microassembly, microparts must be fixed or locked by specific mechanical structures. For example. the Zyvex snap-fastener and its corresponding socket [60] are composed of a 
pair of patented lock mechanisms which can fix $2 \frac{1}{2}$ D Silicon MEMS components with the help of a microgripper. The snap-fastener enables increased assembly tolerance and final alignment of microparts into desired positions.

In the serial microassembly process, only one micropart at a time is moved by the combination of: 1) a high precision manipulator with feedback from axis position sensing, 2) advanced sensory feedback from the assembly scene such as microscope vision feedback for adjusting and aligning microparts to their desired locations and 3) a microgripper for grasping and manipulating microparts. However, serial microassembly has throughput limitations because only one microcomponent is assembled at once [59].

In order to overcome throughput limitations, parallel microassembly has been proposed to handle a large number of microparts simultaneously. In past work, two fundamental approaches have been studied: one is dependent on the transfer in parallel between two wafers of micropart arrays, which is called deterministic parallel microassembly; the other, named stochastic parallel microassembly, utilizes force fields, such as fluids or vibrational energy to align $d$ a randomly oriented arrays of microcomponents [58]. The latter approach is similar to self-assembly bottom up manufacturing approaches found in nanotechnology [58]. Even though parallel microassembly provides a faster assembly rate than serial microassembly, the yield losses are relatively larger than serial microassembly, which may make this approach less feasible in practical applications. 


\section{CHAPTER 3}

\section{MICROROBOTS}

The first type of microrobot investigated during this research was an untethered, magnetically driven microrobots, that participated at the Mobile Microrobot Challenge 2017 of IEEE, and which has application for manufacturing and medicine

\subsection{Introduction - MMC}

The Mobile Microrobot Challenge is held annually by the Institute of Electrical and Electronics Engineers (IEEE) Robotics \& Automation Society (RAS) Micro/Nano Robotics \& Automation Technical Committee (MNRA) [61]. The aim of the MMC is to encourage researchers from all over the world to explore the tasks of autonomy, accuracy, and assembly with microrobots which must fit in a virtual cube measuring 500 microns on each side. The MMC has three different events: a) autonomous mobility and accuracy challenge, b) microassembly challenge, and c) MMC showcase and poster session. The first two challenges are inspired by analogous applications for medicine and manufacturing, including closed-loop positioning and precision motion control for drug delivery applications, and microassembly of MEMS components.

The Next Generation System (NGS) Lab at University of Louisville participated in MMC 2017 held in Singapore using a conical magnet to generate a focused magnetic field for microrobot actuation in a specific arena. The technique was originally proposed and investigated by Torres [62, 63], and was also used at MMC 2014 and 2015 
The focused magnetic field has a sharp gradient close to the cone tip, so that it generates a powerful attractive force, and actuates magnetic material items and drives them to along desired trajectories on the competition substrate. During my research I used 250 microns diameter chrome-steel spheres and a 250 microns neodymium cubical magnet as microrobots for first and second challenges, respectively.

\subsection{Automation for MMC}

For MMC 2017[61], the first challenge required that that microrobots must navigate between a series of waypoints that are provided dynamically at the start of the event. The competition arena and corresponding waypoints are shown in Figure 3.1 (a). There are two different colorful waypoints: green (targets) and red (obstacles). The goal is to actuate microrobots along a path which covers all the targets and avoids all the obstacles. Motion needs to be automated and completed in as short a time as possible, and no longer than 2 minutes. The physical dimensions of the arena for the first challenge was $3.25 \mathrm{~mm}$ x $2 \mathrm{~mm}$, with 28 waypoints at $250 \mu \mathrm{m}$, while the computed distance between two closed waypoints computed between each circle center was $500 \mu \mathrm{m}$. Figure 3.1 (b) depicts a sample of a possible path to cover all the targets and miss all the obstacles. Figure 3.1 (c) depicts situations that may arise during robot motion, such as overlapping of the robot onto 2 targets (acceptable), overlapping between the robot and an obstacle (unacceptable), and no overlap (acceptable). 


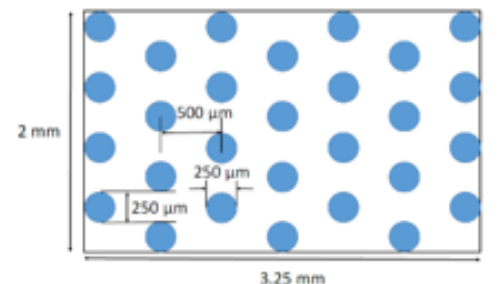

(a)

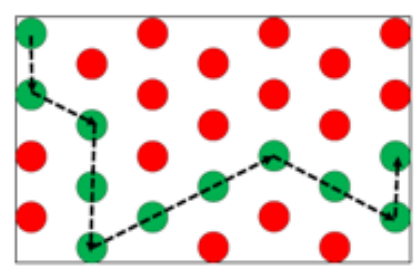

(b)

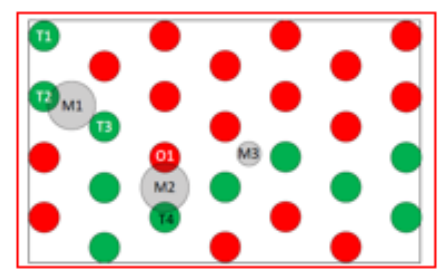

(c)

Figure 3.1. MMC arena definition (a), an MMC path sample (b), and definition of hit and $\operatorname{avoid(c)~[64]~}$

In order to drive the magnetic microrobot, a custom driver was configured using two motorized Newport MFA-CC linear stages, stacked to form an X-Y positioner. A conical permanent magnet was then fixed on the top of the linear stage. A microscope imaging system composed of an Edmund EO-1312C camera associated with VZM 100i zoom imaging lens was locked in a coarse/fine lens mount. Finally, an arena manual positioner was setup by three Thorlabs PT1 manual linear stages moving in X-Y-Z axes. We developed programs used for automation of microrobot motion using National Instruments LabVIEW Vis and USB interface. A joystick and keyboard interface were used for manual intervention by an operator. The MMC hardware system is shown in Figure 3.2. 


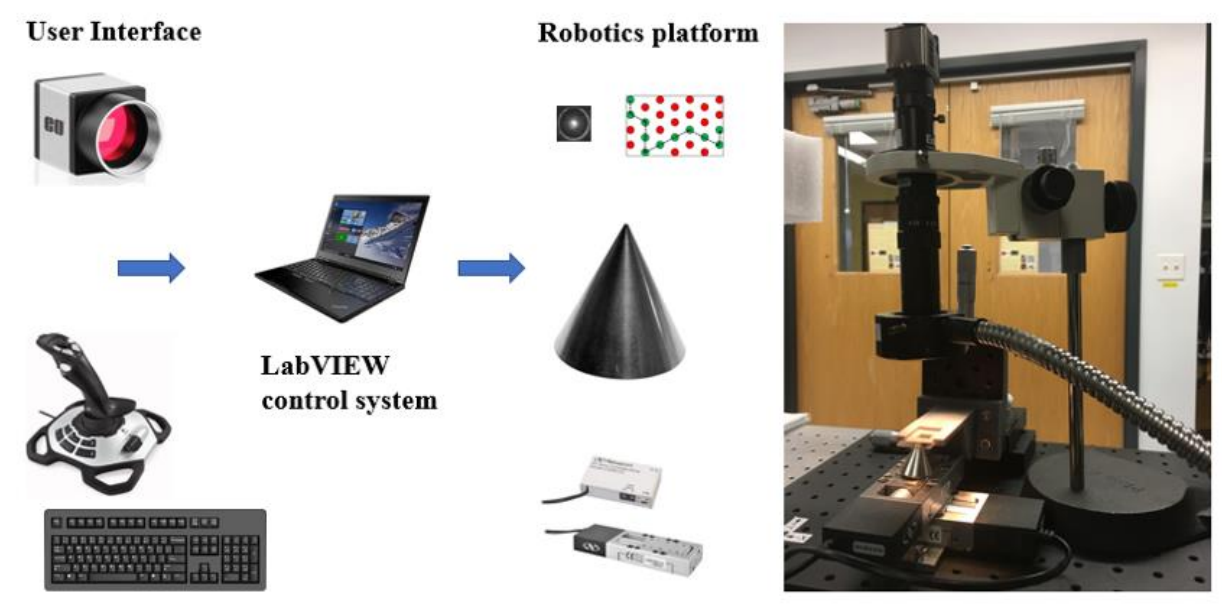

Figure 3.2. MMC2017 hardware setup and corresponding components

The main interface for driving the microrobot to move in the area is presented in

Figure 3.3. It includes a manual control for finding the starting point, waypoints coordinates recorder, and the path control panel for clicking the waypoint to generate the path covering all the targets. After choosing the desired waypoints one by one, by clicking the "Auto trace" button, the microrobot will move in the area automatically following the generated path.

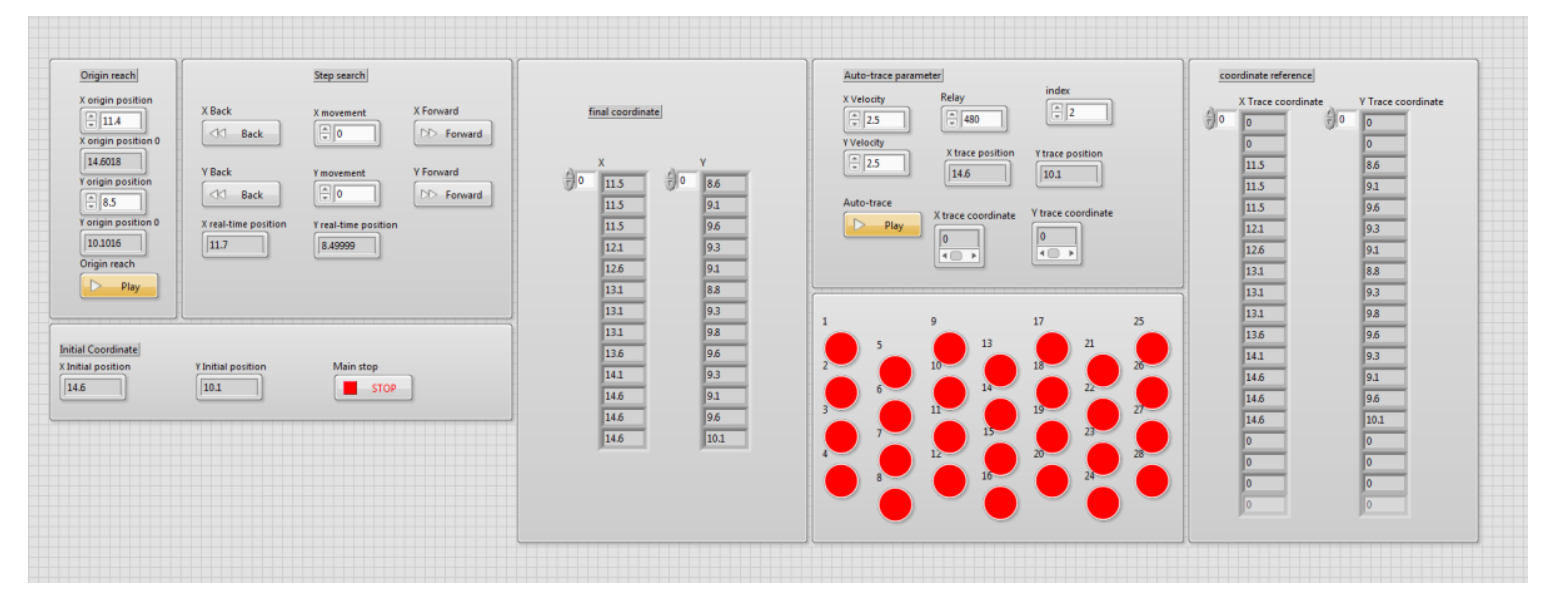

Figure 3.3. MMC2017 first challenge main interface 
In Figure 3.4, there were three different tasks I carried out in the first challenge. Eventually, I earned second place in the competition with other three teams.

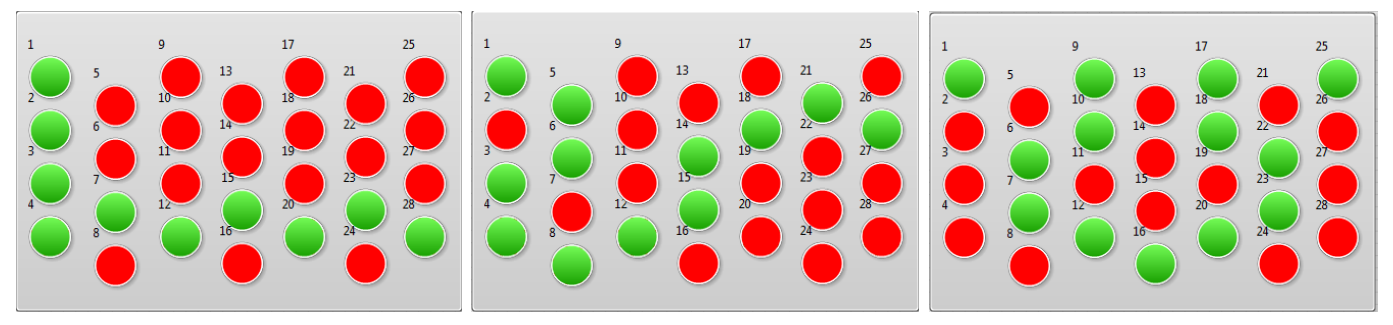

Figure 3.4. Three tasks involved in the first challenge

For the microassembly challenge, the goal was to assemble microparts (triangles with $350 \mu \mathrm{m} \times 200 \mu \mathrm{m}$ side-by-side) at the end of a thin channel. The score was calculated by how many triangles were assembled, and by what methods. Figure 3.5 shows the dimensions (in $\mu \mathrm{m}$ unit) of the arena, and 4 scores achieved by assembling triangles as shown.

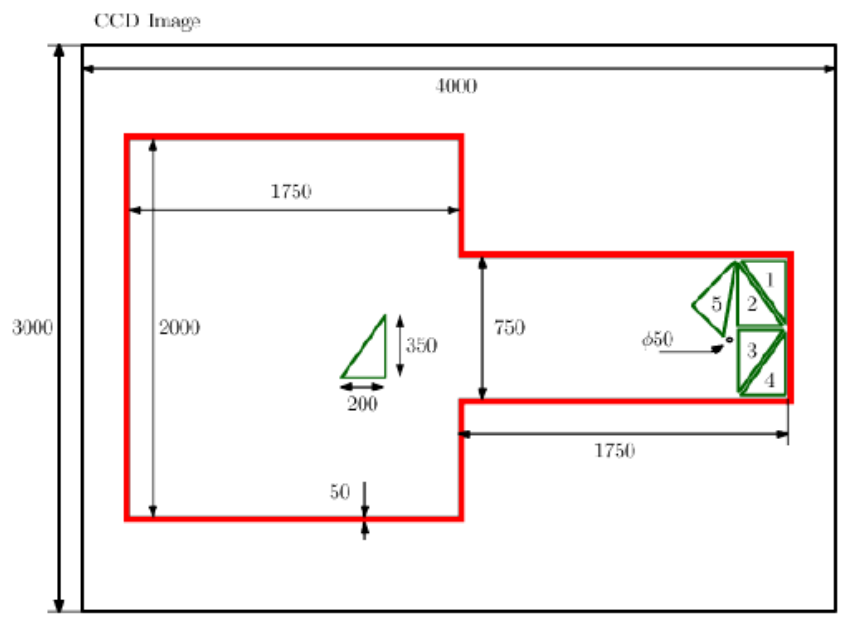

Figure 3.5. Area dimensions and sample of assembly for the second challenge

For the manipulation operation, I preferred to use a keyboard to control the manipulator, because the joystick was too sensitive to operate controllably. According to 
the properties of the keyboard, I created the main interface in Figure 3.6, which have 8 different directions of step control and 7 specific points of fast movement control. After setting up velocity and increment size, the microrobot can be driven to adjust the microparts to proper configurations, and then move them to the desired position and assemble them together.

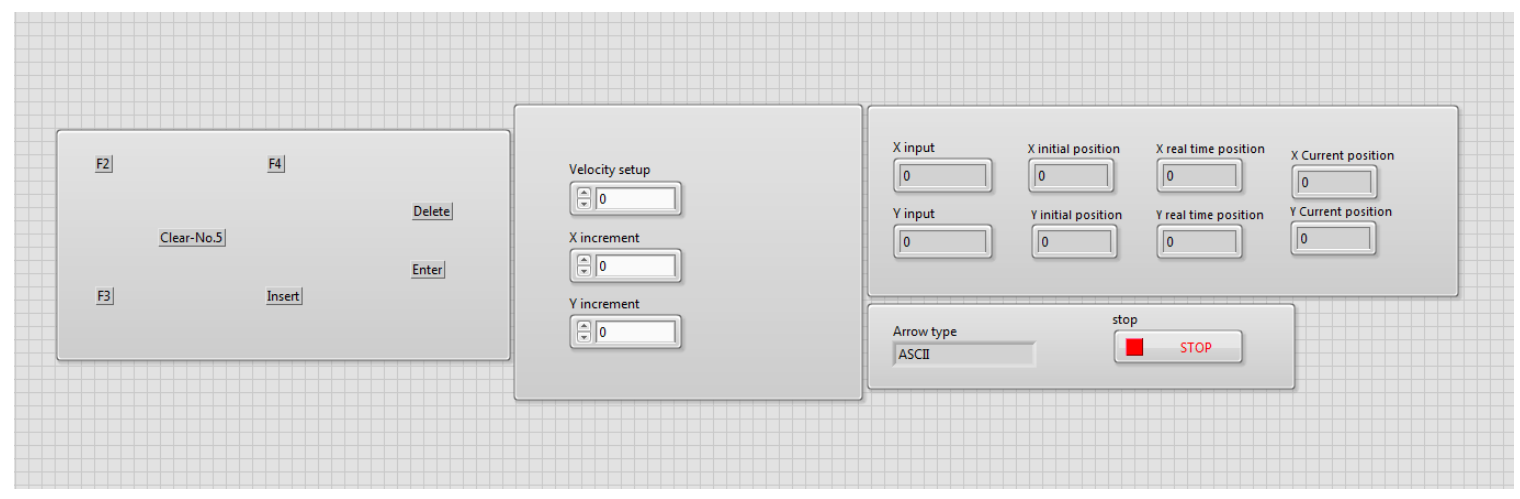

Figure 3.6. MMC2017 second challenge main interface

\subsection{Assembled MEMS Microrobots}

Besides the microrobots applied in MMC, I also did the research on two kinds of assembled MEMS microrobots: AFAM and sAFAM. AFAM is a microassembled robot for nanoscale applications which is composed of a cantilever actuated in 4 degrees of freedom: X, Y, and Yaw (in-plane); Pitch (out-of-plane). The dimensions of AFAM are $3 \mathrm{~mm} \times 1.5 \mathrm{~mm} \times 1 \mathrm{~mm}(\mathrm{XYZ})$, and the workplace operated at $50 \mu \mathrm{m} \times 50 \mu \mathrm{m} \times 75 \mu \mathrm{m}$ (XYZ) [65] as shown in Figure 3.7. AFAM structure design is based on Zyvex snapfastener and a corresponding socket, which can stably lock the out-of-plane microstructure standing onto the substrate. The AFAM cantilever arm is connected to a snap-fastener structure with a flexure spring as shown in Figure 3.8. Designs of the X-Y stages design are based on 4 chevron-electrothermal-beam actuators, which are driven by 
applied electrical power. The AFAM arm and stages were fabricated on Silicon on Insulator (SOI) substrate, whose device layer is 100 microns, by using standard lithographic processes for microfabrication, and utilizing the 3D microassembly process to assemble AFAM arm in a vertical standing position [65]. After fabrication, the AFAM arm is fixed by a tether on the substrate, released by breaking the tether, and picked up by corresponding single microjammer. Next, I rotated the AFAM arm in 90 degrees, in the final step, I vertically assembled the AFAM arm to create the 3D microstructure by using a compliant snap-fastening method. A fiber cable was applied to connect the AFAM arm to the cable drive stage. By actuating two basement stages, the AFAM cantilever arm can be operated in X-Y-Yaw (in-plane) and Pitch (out-of-plane).

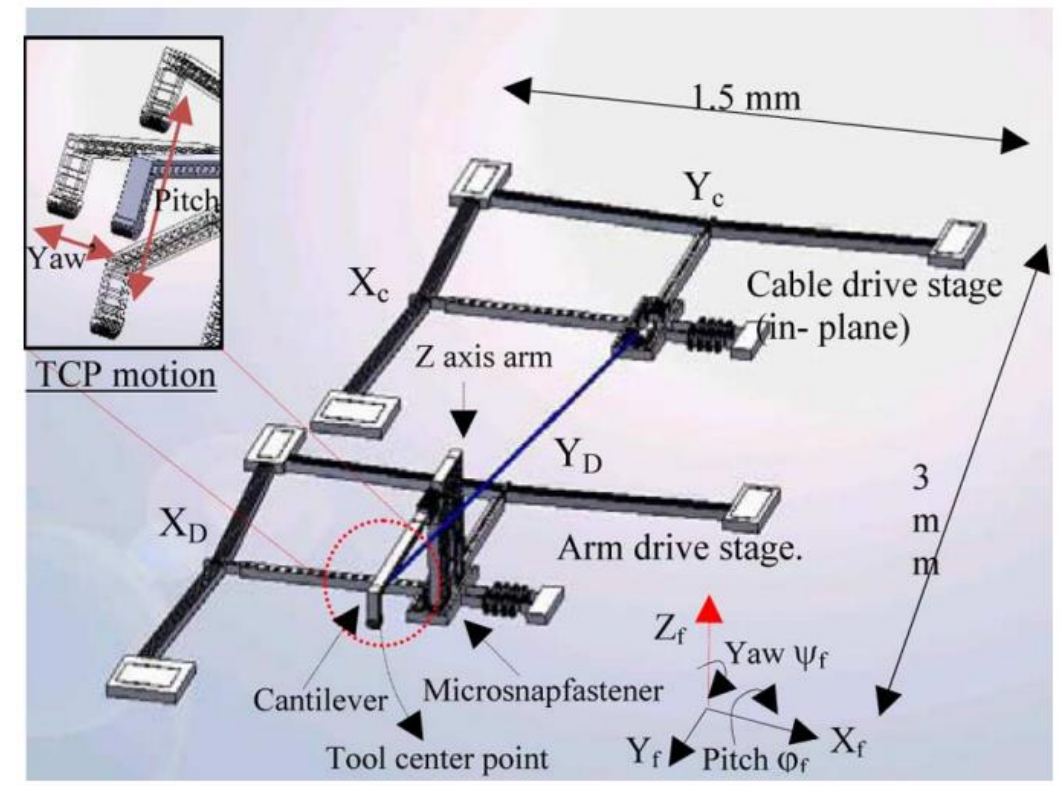

Figure 3.7. AFAM model and dimension[65] 


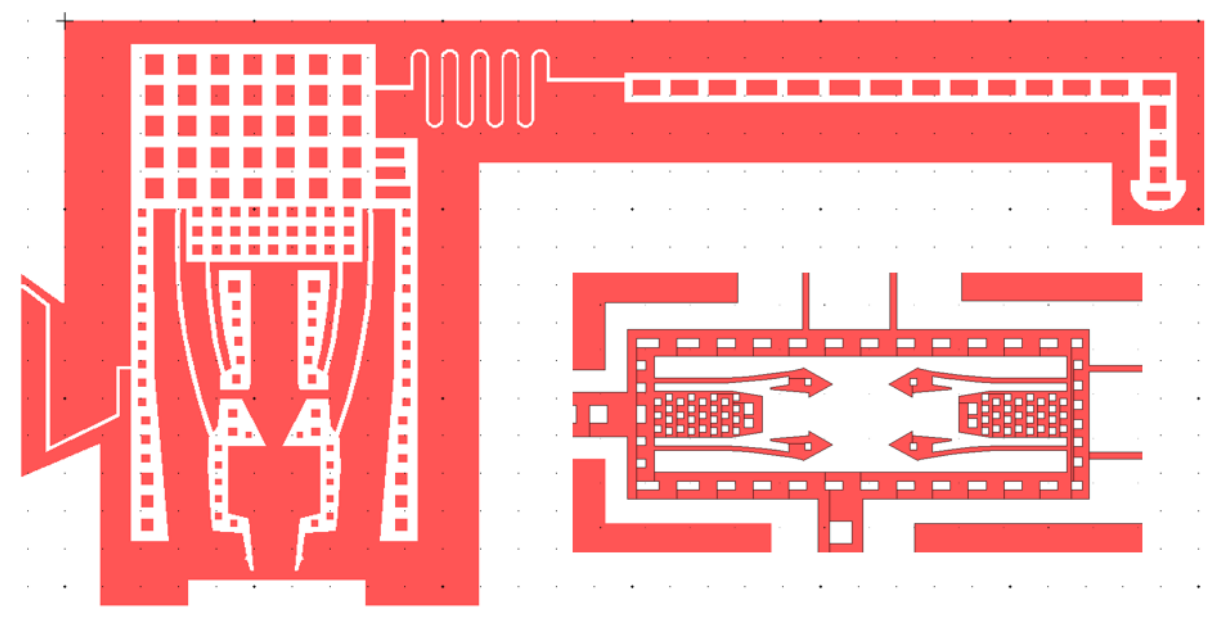

Figure 3.8. AFAM arm with Zyvex snap-fastener and socket

Due to the challenge and complexity of fabricating the fiber cable for driving AFAM arm movement, a new type of 3D microrobot, called sAFAM, has been developed to improve fabrication precision and to reduce assembly process complexities. SAFAM was designed to replace the fiber cable traction system with a unibody arm which can be assembled into the in-plane X-Y stages shown in Figure 3.9. The sAFAM design was analyzed using Finite Element Analysis (FEA), and then fabricated and tested to confirm a 3D workspace of approximately $22 \mu \mathrm{m} \times 47 \mu \mathrm{m} \times 185 \mu \mathrm{m}$. Meanwhile, the fabrication of sAFAM was similar to the standard lithographic microfabrication processes of AFAM. During the assembly process of sAFAM a the dual microjammer was utilized to break two tethers which fix the microrobot arm to the SOI device layer. The AFAM and sAFAM assembly process is discussed in detail in Chapter 4. 


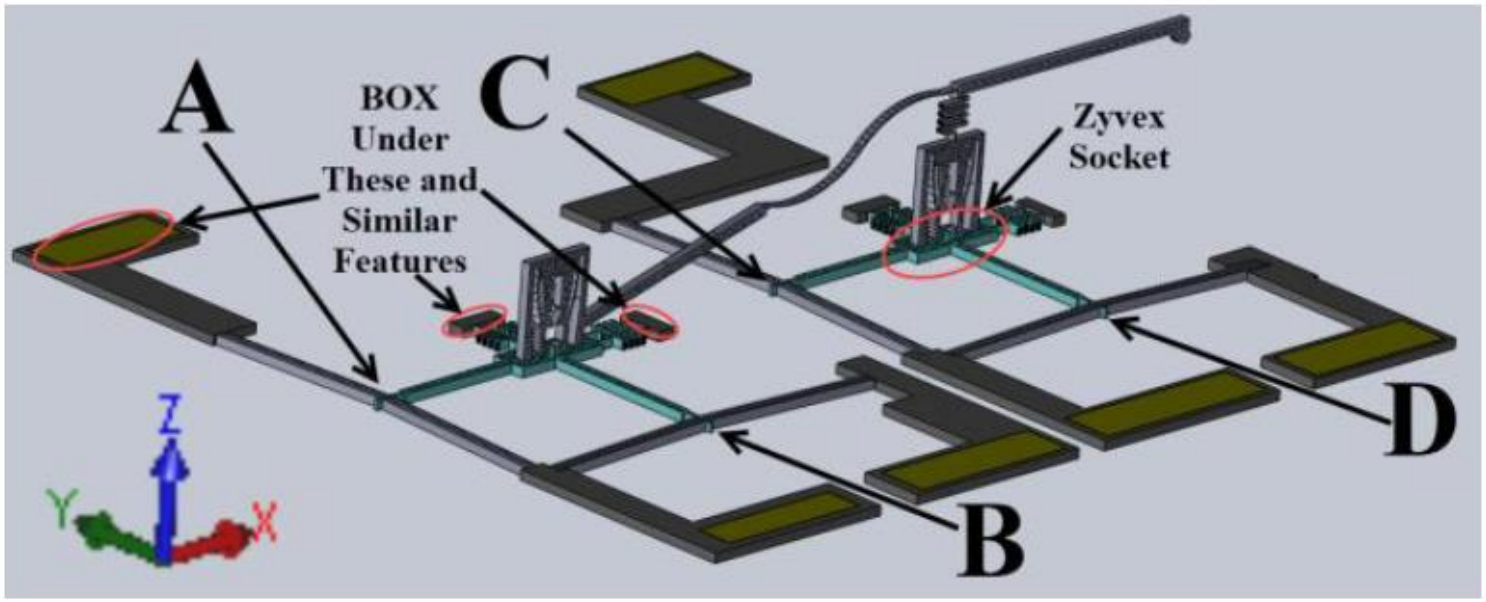

Figure 3.9. 3D model of sAFAM from SolidWorks ${ }^{\mathrm{TM}}$ 


\section{CHAPTER 4}

\section{NEXUS MICROASSEMBLY SYSTEM DESIGN}

In this chapter, I will discuss the robotic microassembly system (NeXus) which I designed and implemented during this research. The NeXus contains accurate micropositioners, microscopes, and controllers as well as programming driven via LabVIEW. In addition, before the robotic microassembly system was physically implemented, a virtual one was simulated using LabVIEW. Moreover, there are some specific processes which are needed to assist the operation of a robotic microassembly system, such as rotation centering and visual servoing, which this chapter will also discuss. Finally, experimental results by pick-rotate-place automation to assemble microrobots will be discussed to show the feasibility of NeXus in practice.

\subsection{Design and simulation of NeXus}

The simulation of a visual robotic microassembly system is useful for setting up the corresponding system with accurate micro-positioners, microscopes, and controllers. The LabVIEW is available and practical for designing the programming for simulation of a visual robotic microassembly system (shown in Figure 4.1). 


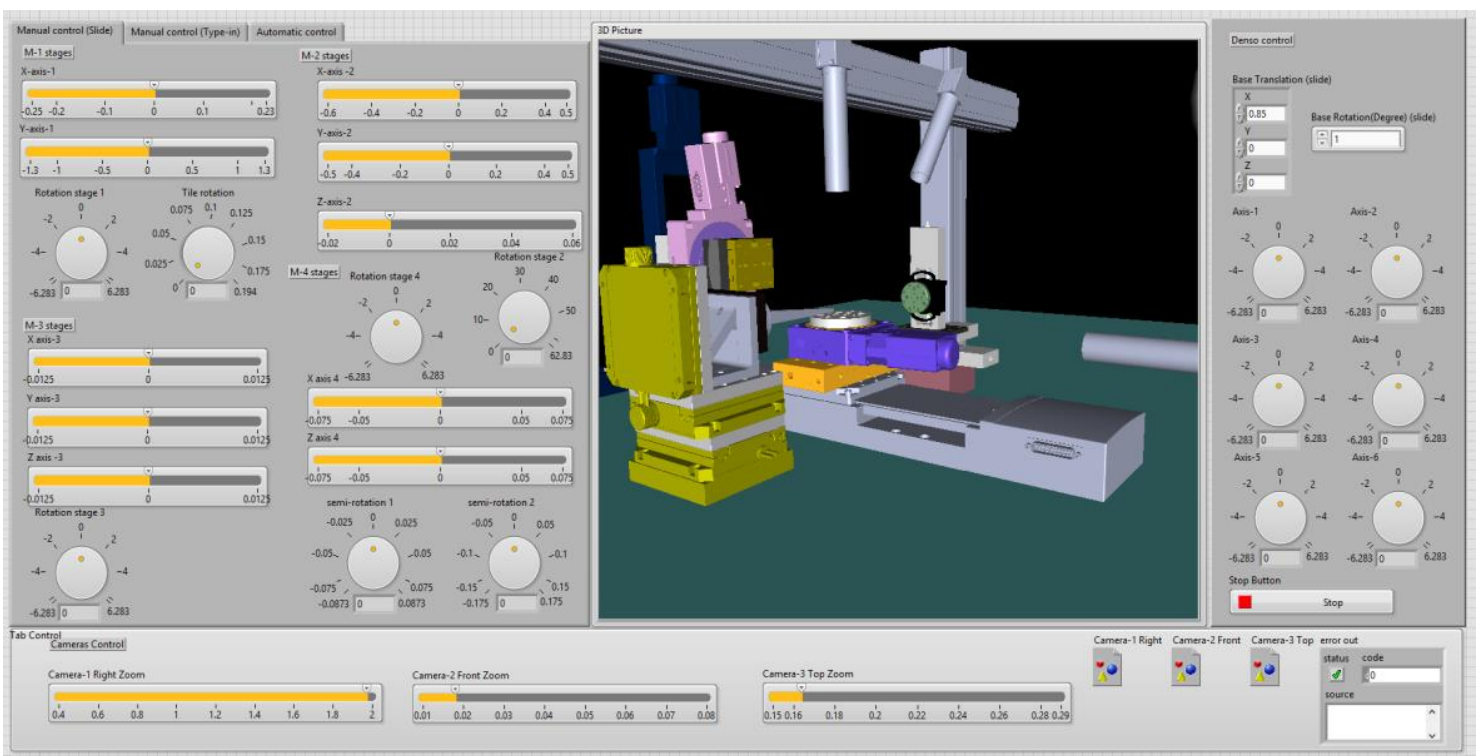

Figure 4.1. Main interface of NeXus by LabVIEW

The whole NeXus consists of 4 manipulators and 3 microscopes distributed shown in Figure 4.2,

- Manipulator 1 (M1): This is a sample carrier stage which has a die holder as the end-effector to hold the sample. Meanwhile, it has two linear stages and one rotation stage to set up an $\mathrm{X}-\mathrm{Y}$-Th stage arrangement with 3 degrees of freedom (DOF).

- Manipulator 2 (M2): This is a fast and precise manipulator with an X-Y-Z stage and an additional rotation stage, which has a 4-DOF with X-Y-Z-Th. It can be used for precise tasks with an end-effector mounted microjammer or microgripper to complete the pick-rotate-place operation for microrobot assembly tasks. 
- Manipulator 3 (M3): This is a light-duty manipulator with 3 linear stages and a rotation stage, which is similar to $\mathrm{M} 2$. It can be applied to additional operations assisting M2 to accomplish further assembly applications.

- Manipulator 4 (M4): This is a heavy-duty manipulator with two large linear stages for $\mathrm{Y}-\mathrm{Z}$ axes. In addition, there is a rotation stage and two tilt stages mounted on linear stages to form a Y-Z-Th-Tilt, 4-DOF manipulator. It can carry two tools at the same time due to its long-range $\mathrm{Y}$ and $\mathrm{Z}$ axes.

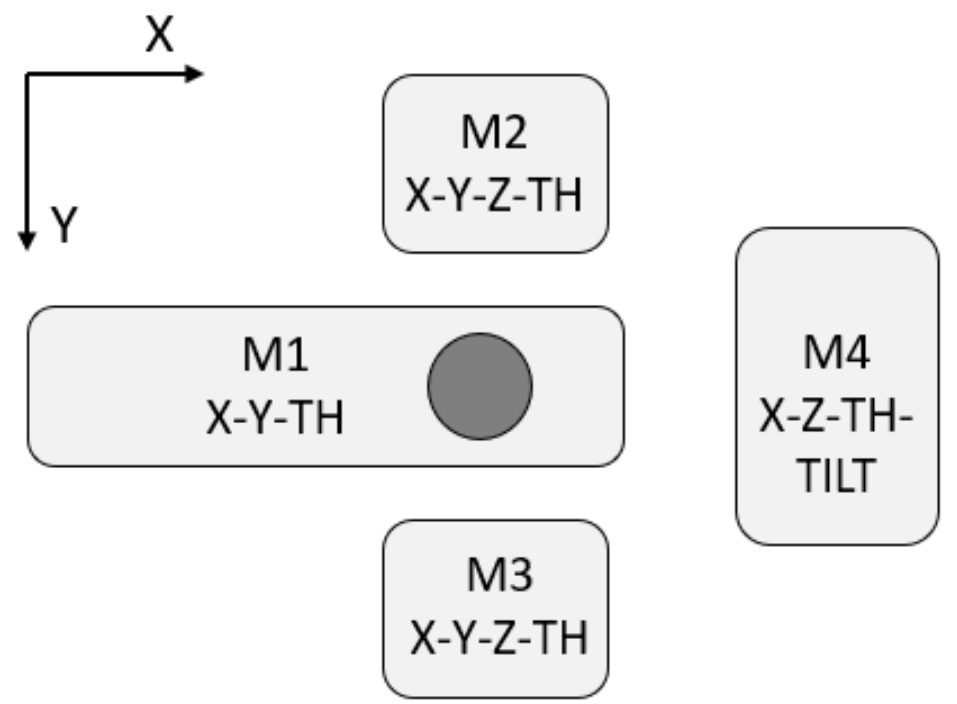

Figure 4.2. Distribution of 4 manipulations in LabVIEW simulation of NeXus

To construct a simulation environment in LabVIEW, the first step is to load the actuator stages' CAD files in a program such as SolidWorks ${ }^{\text {TM }}$, then modify and split them into individual movable components exported as VRML format files as depicted in Figure 4.3. In that way, the parts imported in LabVIEW as VRML files can move individually. In the block diagram, each manipulator connects several components together in a serial manner, although they actually operate in parallel as a collection of 
subVIs. Using these methods, the whole microassembly system simulation model can be built and then operated in both manual and automatic modes. The detailed VI programs for the NeXus microassembly system are referenced in APPENDIX A.
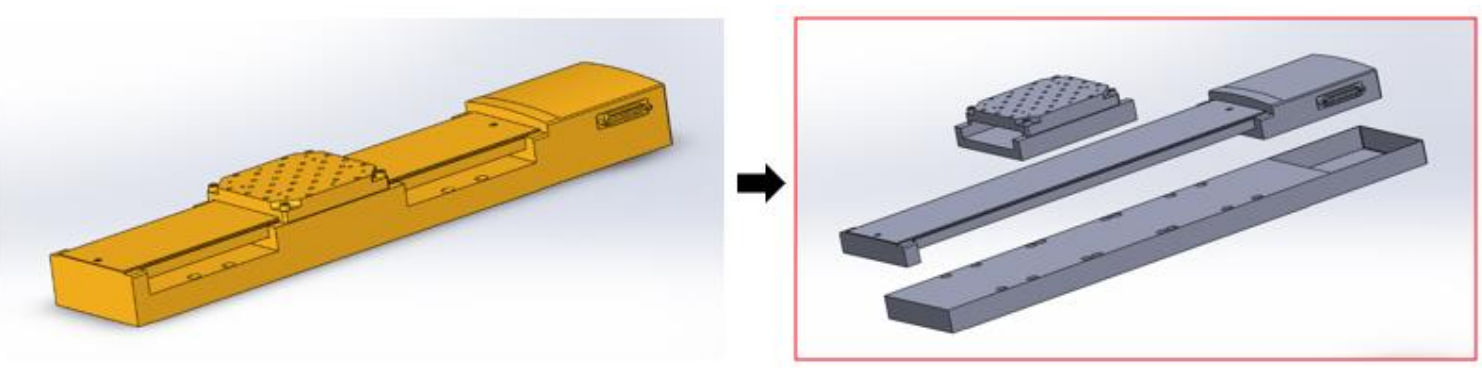

Figure 4.3. Steps necessary to split a linear stage CAD file into several movable parts

\subsection{NeXus hardware setup}

Based on the LabVIEW simulation of NeXus, a partial platform of NeXus with M1 and M2 has been set up for specific microrobot assembly experiments. The recent robotic microassembly system consists of M1 and M2 as well as three different position microscopes with illumination devices from vertical-horizontal-side views monitoring the processing of microrobot assembly (as shown in Figure 4.4). 


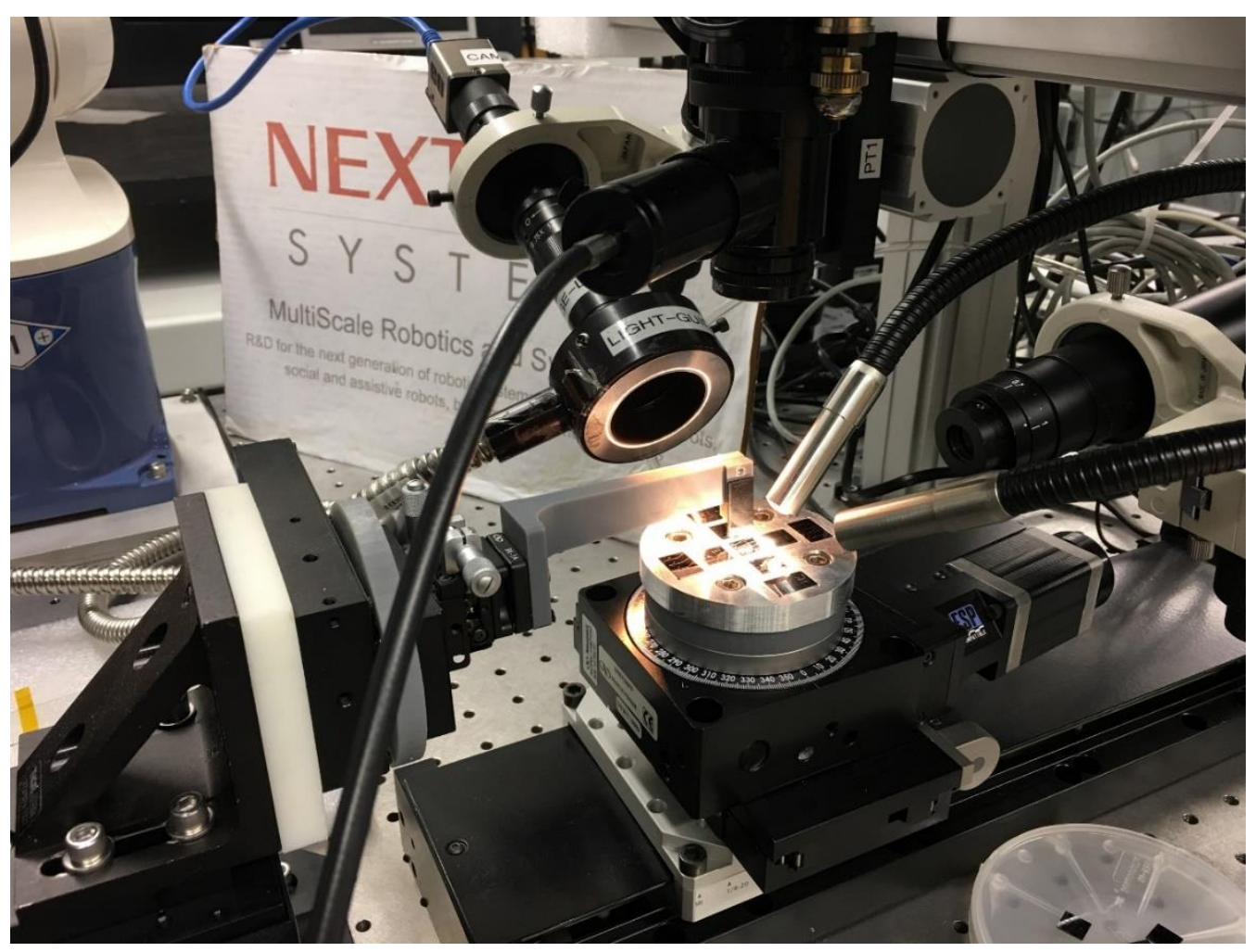

Figure 4.4. Recent physical configuration of the NeXus robotic microassembly system

Manipulator 1 consists of X motorized stage (ILS250CC), Y motorized stage (443 series \& LTA-HS), motorized rotation stage (URS75BPP), and die holder as shown in Figure 4.5. The die holder was custom machined for carrying several dies with two different dimensions, $10 \mathrm{~mm} \times 10 \mathrm{~mm}$ and $20 \mathrm{~mm} \times 10 \mathrm{~mm}$, and these dies are fixed to the holder by vacuum. 

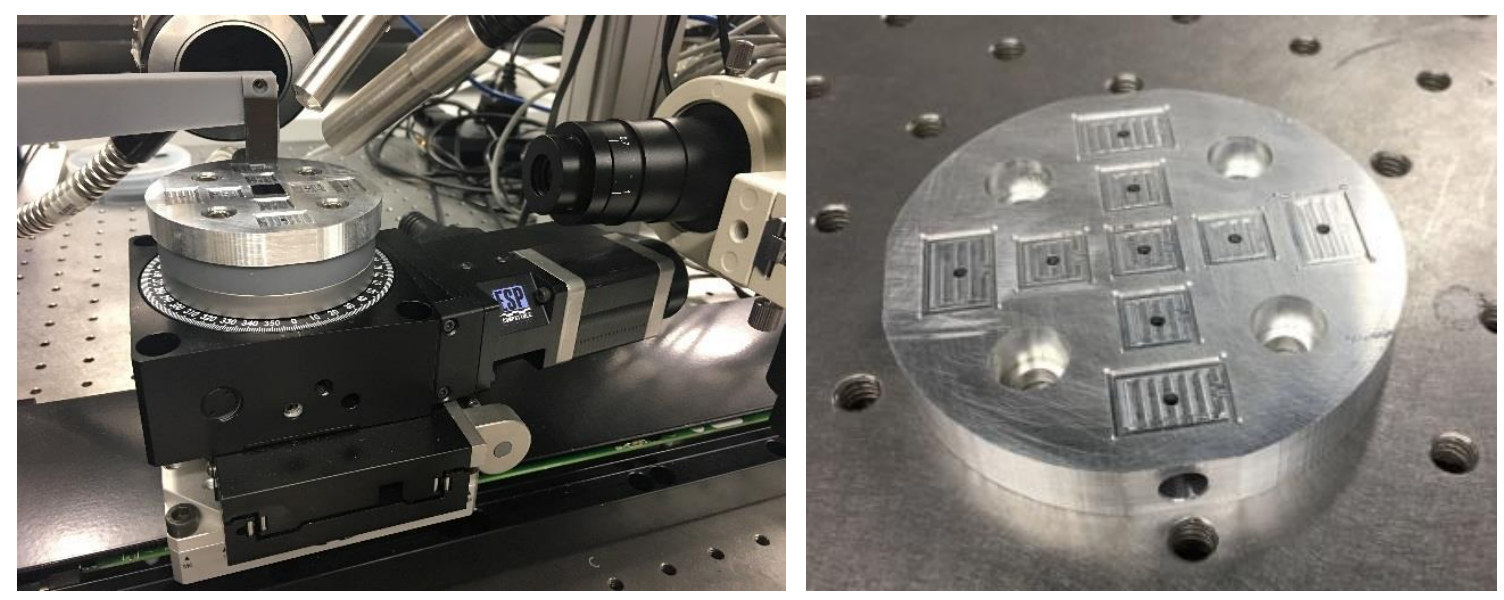

Figure 4.5. Manipulator 1 and die holder

To configure Manipulator 2 shown in Figure 4.6, we combined a motorized X-YZ stage (VP-25XA-XYZR) combined with a motorized rotation stage (PR50CC), as well as a manual $\mathrm{X}-\mathrm{Y}$ translation stage which is for rotation centering. The end-effector is connected to a kinematic base (BK-1A) which has two parts attached to each other by magnetic force, and it can be separated freely. Consequently, one part is fixed on the translation stage, which the other can be fixed with an end-effector and is easy to replace with other end-effectors. In addition, several intermediate parts were fabricated by 3D printing and used to connect and align the manipulator stages. 


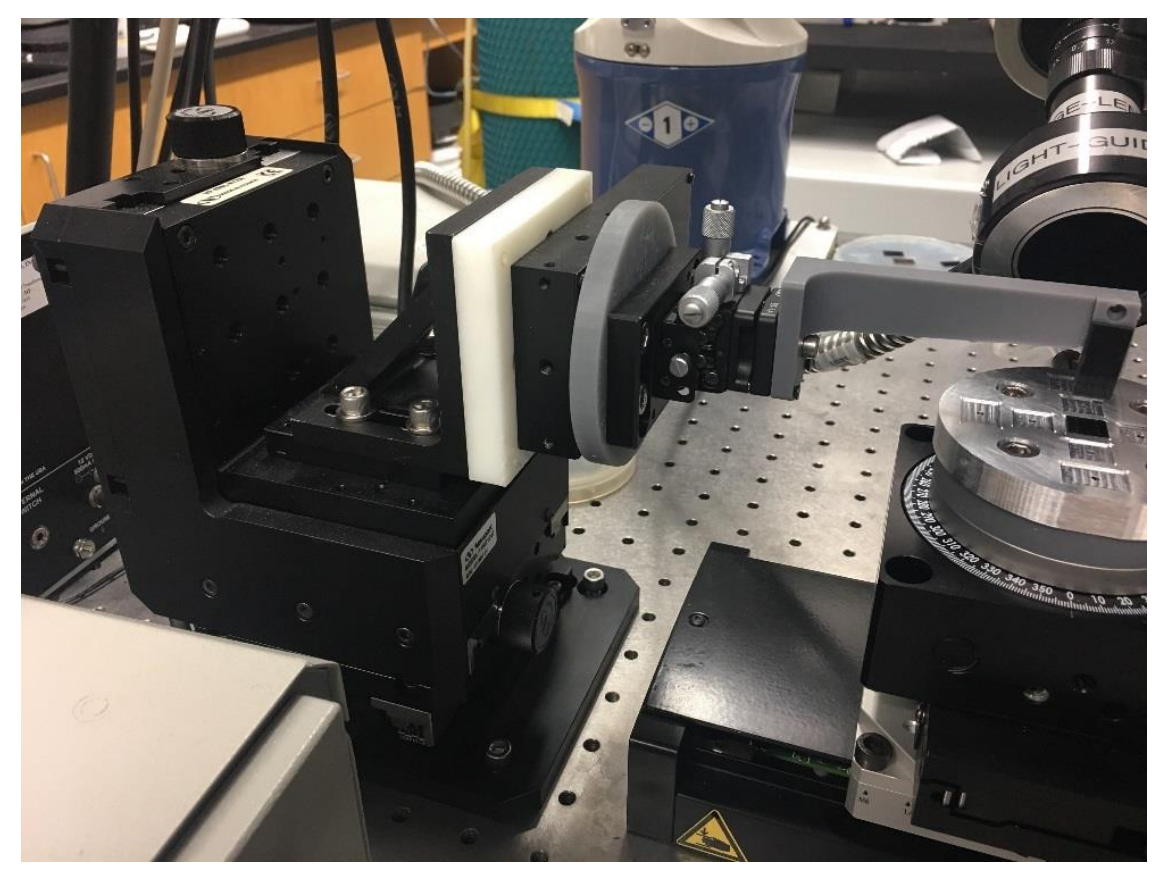

Figure 4.6. Manipulator 2 with end-effector mounted microjammer

The NeXus feedback monitoring system is composed of 3 imaging systems from vertical, horizontal, and side views to provide image feedback from the microstructures assembly process. In Figure 4.7, the vertical microscope is composed of two Thorlabs PT1 translation stages, an Edmund EO-3112C camera, a Dolan-Jenner Fiber-Lite DC950 fiber optic illuminator, and a QIOPTIQ Optem zoom lens combined with its stepper motor controller. The vertical imaging system can be translated in $\mathrm{X}-\mathrm{Z}$ directions to broaden the field of view. Also, the stepping motor controller can adjust the zoom lens for zoom-in and zoom-out with different speeds. 

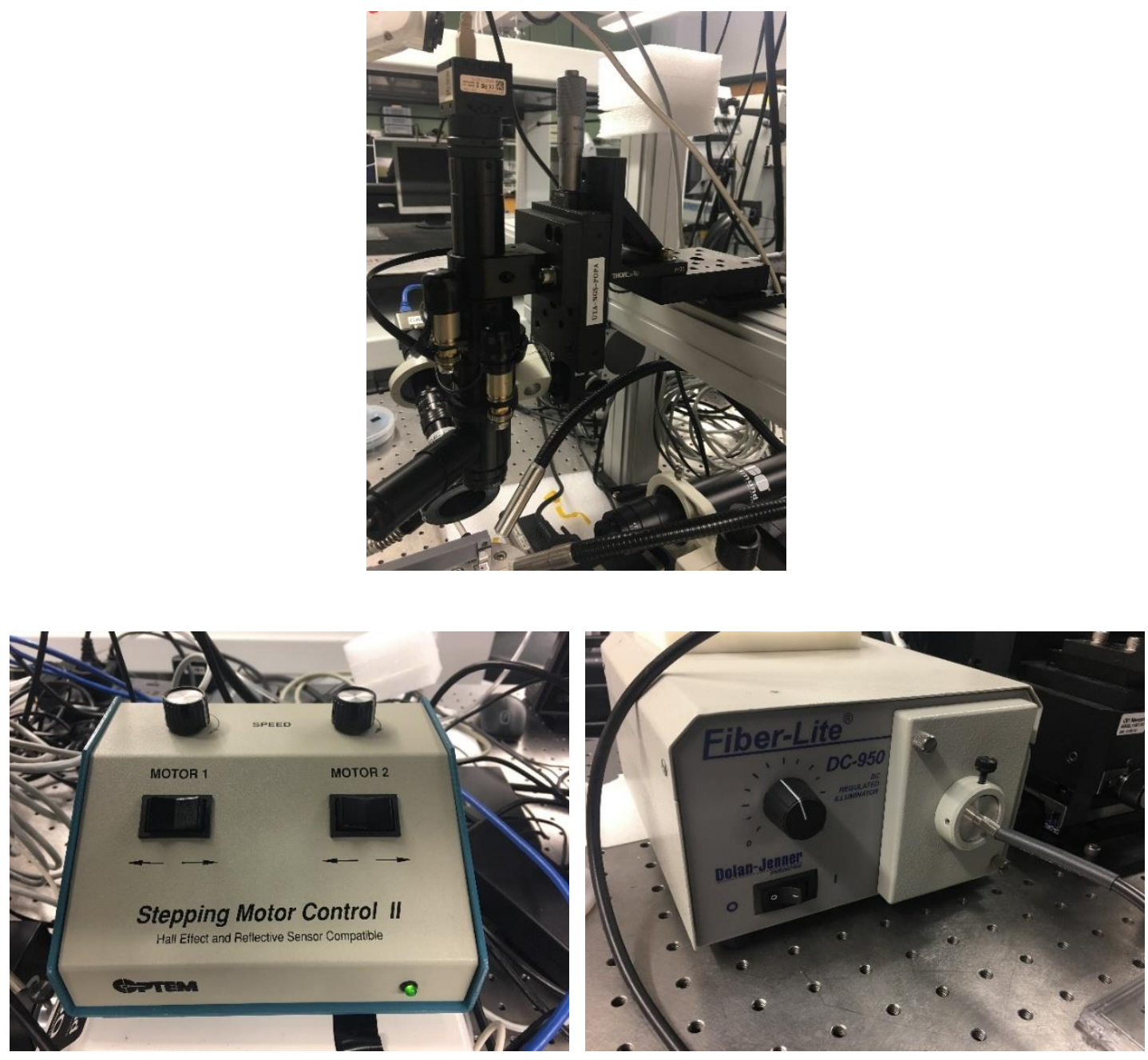

Figure 4.7. Vertical imaging system with zoom lens, stepping motor control, and illuminator

The horizontal imaging system shown in Figure 4.8, can be adjusted in X-Y-Z-Th with three Thorlabs PT1 translation stages and rotation and coarse/fine movement along with the Edmund lens mount. A gooseneck optic fiber associated with the Edmund MI150 illuminator can provide much better illumination for the horizontal microscope, especially for the process of rotation centering. The Edmund VZM 450 zoom imaging lens mounted with the Edmund EO-0413M camera can provide a sufficiently large and clear field of view during microassembly operations. 

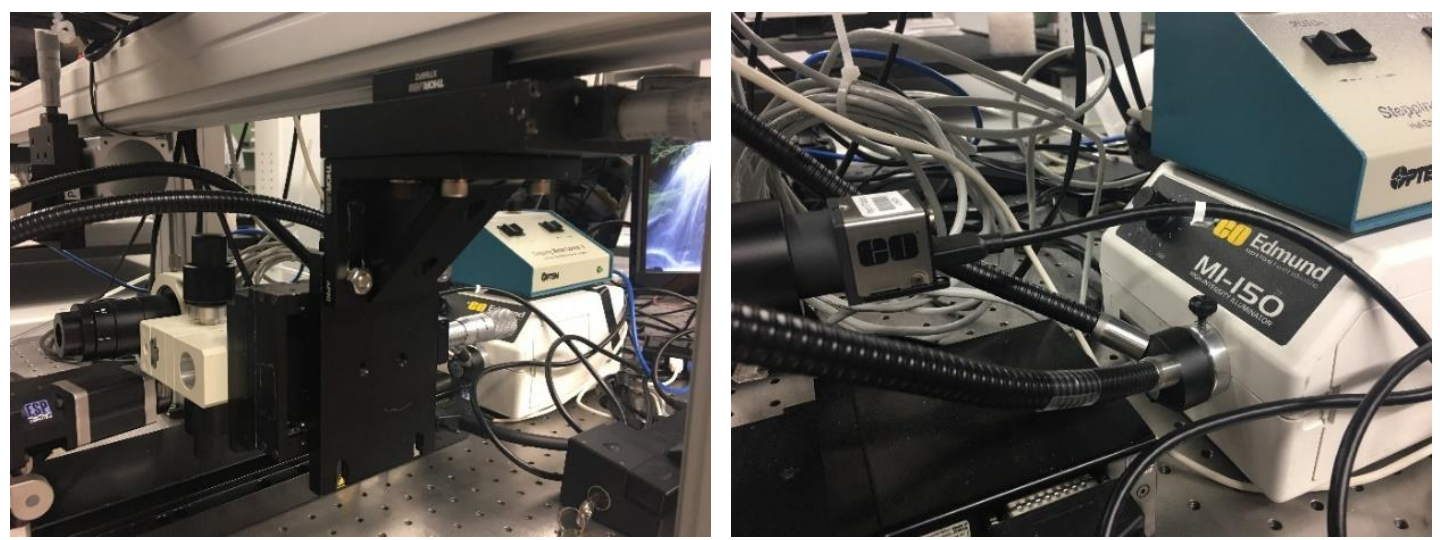

Figure 4.8. Horizontal imaging system and illuminator with gooseneck optic fiber

The side microscope, in Figure 4.9, consists of a PT1 translation stage and an RP01 manual rotation stage (both from Thorlabs) which are used to adjust the image position. Meanwhile, the Edmund EO-1312C camera associated with the VZM 100i zoom imaging lens locked in a coarse/fine lens mount and fixed on a rotational stage can capture side view images and monitor the whole assembly process.
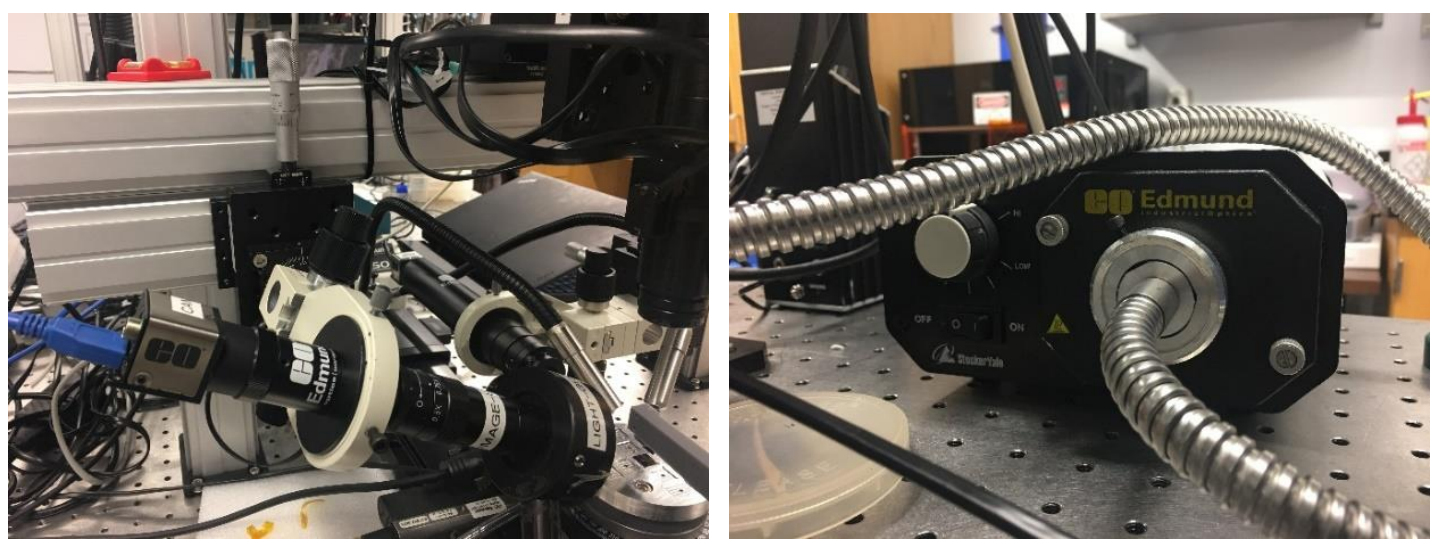

Figure 4.9. Side imaging system and illuminator

To load and unload dies from the die holder, a vacuum tweezer was utilized. The vacuum pen (shown in Figure 4.10) can provide enough vacuum to absorb the die with an EFD tip, and to pick up and drop dies to the desired square areas. 

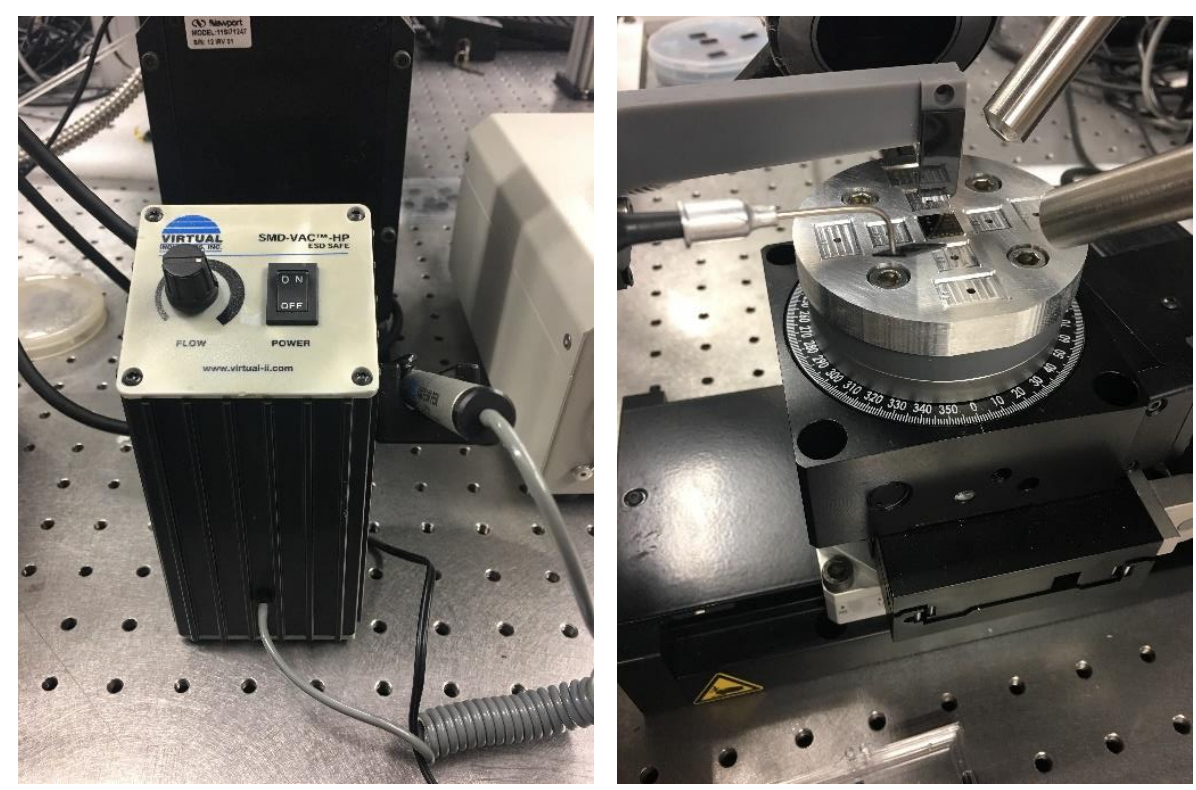

Figure 4.10. Vacuum pen for loading and unloading dies

The motorized stages included in manipulators M1 and M2 are driven by Newport Corporation XPS-Q8 motion controllers, which connect at most 8-external relative motorized stages (shown in Figure 4.11). There are seven motorized stages which communicate with the controller by DB25 Male to DB25 Female cables. Moreover, an Ethernet cable is used to enable communication between the controller and a PC.
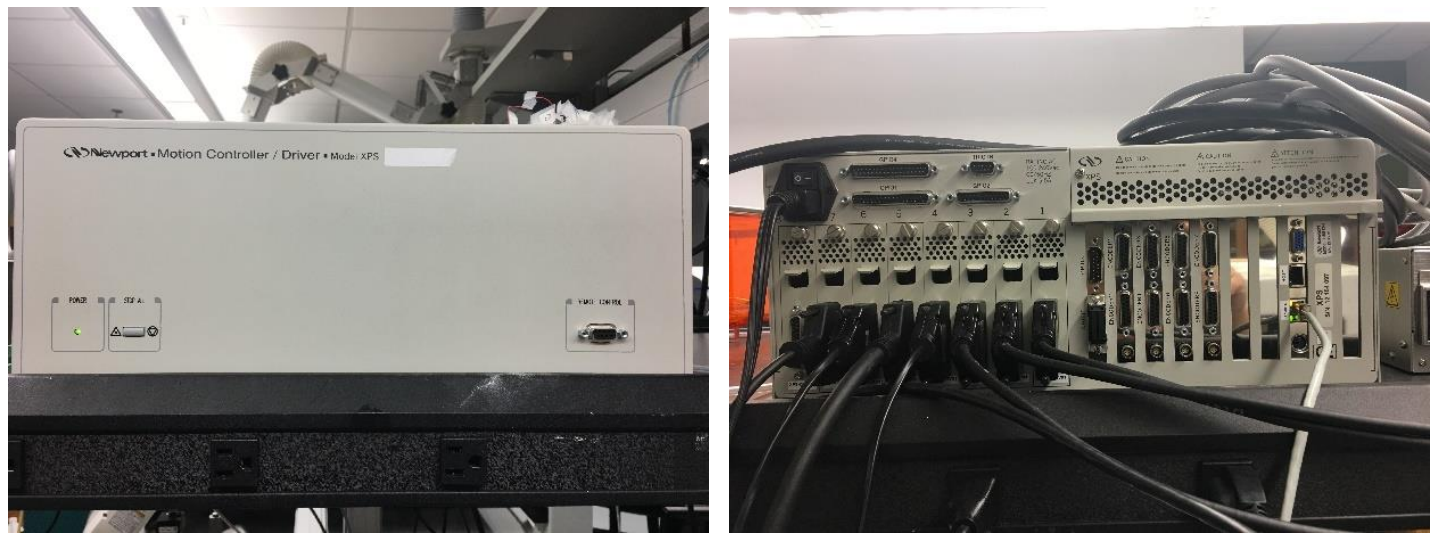

Figure 4.11. Front and back of XPS motion controller 
The actual distribution of M1 and M2 is shown in Fig. 4.12, depicting a top-view of the assembly workspace. Representative parameters for all stages are shown in Table 4.1.

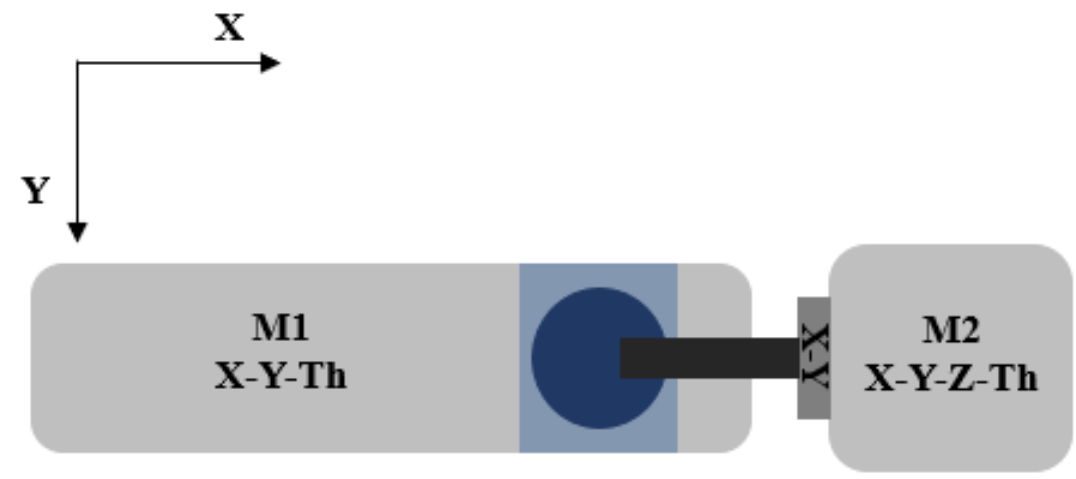

Figure 4.12. Diagram of M1 and M2

Table 4.1. The parameters of all motorized stages for M1 and M2

\begin{tabular}{|c|c|c|c|c|c|}
\hline & ILS250CC & $\begin{array}{c}433+\mathrm{LTA}- \\
\text { HS }\end{array}$ & URS75BPP & $\begin{array}{c}\text { VP-25XL- } \\
\text { XYZR }\end{array}$ & PR50CC \\
\hline $\begin{array}{c}\text { Minimum } \\
\text { incremental } \\
\text { motion }\end{array}$ & $1.0 \mathrm{um}$ & $0.10 \mathrm{um}$ & $0.20 \mathrm{mdeg}$ & $0.01 \mathrm{um}$ & $20 \mathrm{mdeg}$ \\
\hline Travel range & $250 \mathrm{~mm}$ & $46 \mathrm{~mm}$ & $360^{0}$ & $25 \mathrm{~mm}$ & $360^{0}$ \\
\hline $\begin{array}{c}\text { Maximum } \\
\text { Speed }\end{array}$ & $100 \mathrm{~mm} / \mathrm{s}$ & $5 \mathrm{~mm} / \mathrm{s}$ & $40^{0} / \mathrm{s}$ & $25 \mathrm{~mm} / \mathrm{s}$ & $20^{0} / \mathrm{s}$ \\
\hline $\begin{array}{c}\text { Uni- } \\
\text { directional } \\
\text { repeatability, } \\
\text { guaranteed }\end{array}$ & $+/-0.5 \mathrm{um}$ & $+/-0.25 \mathrm{um}$ & $+/-1.0 \mathrm{mdeg}$ & $+/-0.07 \mathrm{um}$ & $+/-15 \mathrm{mdeg}$ \\
\hline $\begin{array}{c}\text { Bi- } \\
\text { directional } \\
\text { repeatability, } \\
\text { guaranteed }\end{array}$ & $+/-1.0 \mathrm{um}$ & $+/-10 \mathrm{um}$ & $+/-6.0 \mathrm{mdeg}$ & $+/-0.07 \mathrm{um}$ & $+/-75 \mathrm{mdeg}$ \\
\hline $\begin{array}{c}\text { Accuracy, } \\
\text { guaranteed }\end{array}$ & $+/-5.0 \mathrm{um}$ & $+/-5 \mathrm{um}$ & $+/-15 \mathrm{mdeg}$ & $+/-1.0 \mathrm{um}$ & $+/-50 \mathrm{mdeg}$ \\
\hline $\begin{array}{c}\text { Load } \\
\text { capacity }\end{array}$ & $250 \mathrm{~N}$ & $191 \mathrm{~N}$ & $200 \mathrm{~N}$ & $35 \mathrm{~N}$ & $10 \mathrm{~N}$ \\
\hline
\end{tabular}


The NeXus microassembly system was set up to assemble microrobots such as the AFAM and sAFAM. It is important to select proper microtools or end-effectors to mount on M2 in order to pick-place microparts mounted on M1. There are two microjammers with single-head and dual-heads applied for AFAM and sAFAM respectively as shown in Figure 4.13.

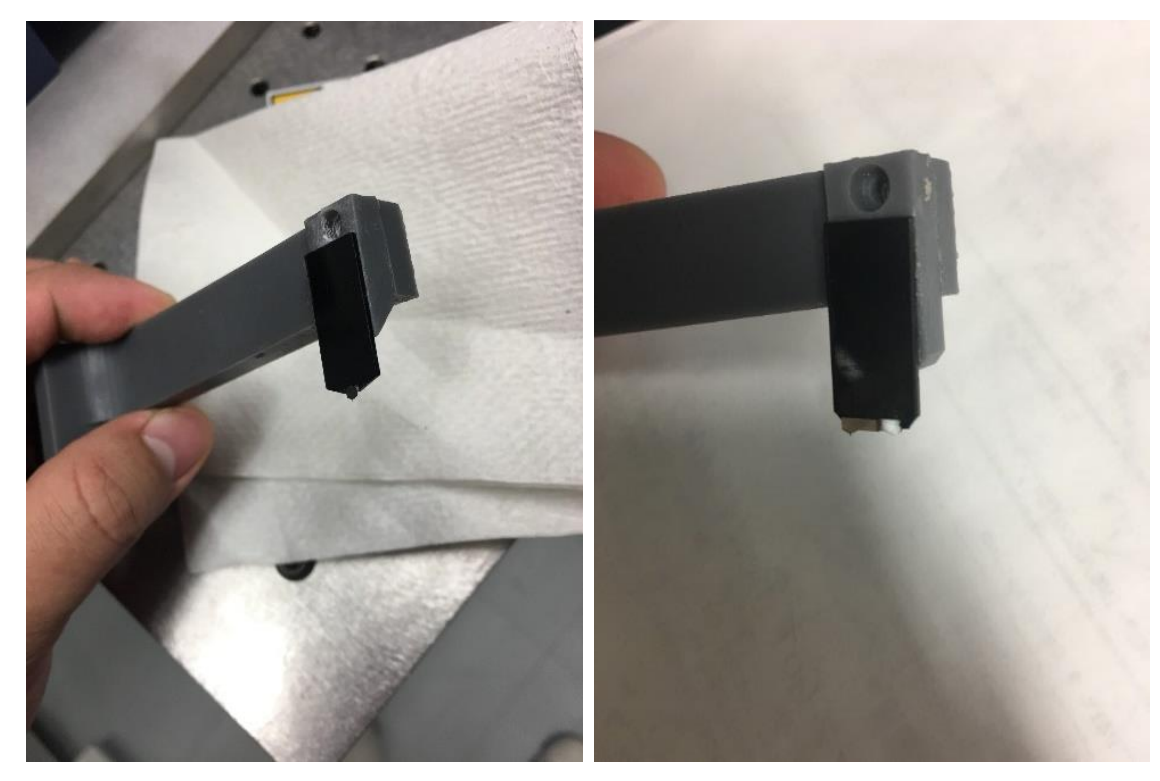

Figure 4.13. Single head and dual heads microjammers mounted on the end-effector

The single-head microjammer is mounted to the end-effector by spraying epoxy adhesive (3M Super 77) on a small area close to the edge of the bottom of the endeffector and then adjusting the M2 with top microscope assistance to achieve the desired position and orientation of the microgripper. Then, the end-effector is brought in contact with the single-head microjammer laid on the wafer taped to the die holder. Finally, the end-effector is lifted relatively quickly off the substrate, and then the jammer assembly is air-dried for several minutes prior to use. Figure 4.14 shows the single-head microjammer mounted on the end-effector. The same method was also utilized for mounting the dualheal microjammer on the end-effector. The only difference is that the top imaging system 
is adjusted in the $\mathrm{X}$ direction to align the dual-head microjammer, because the long size of dual head microjammer is beyond the field of view of the top camera.
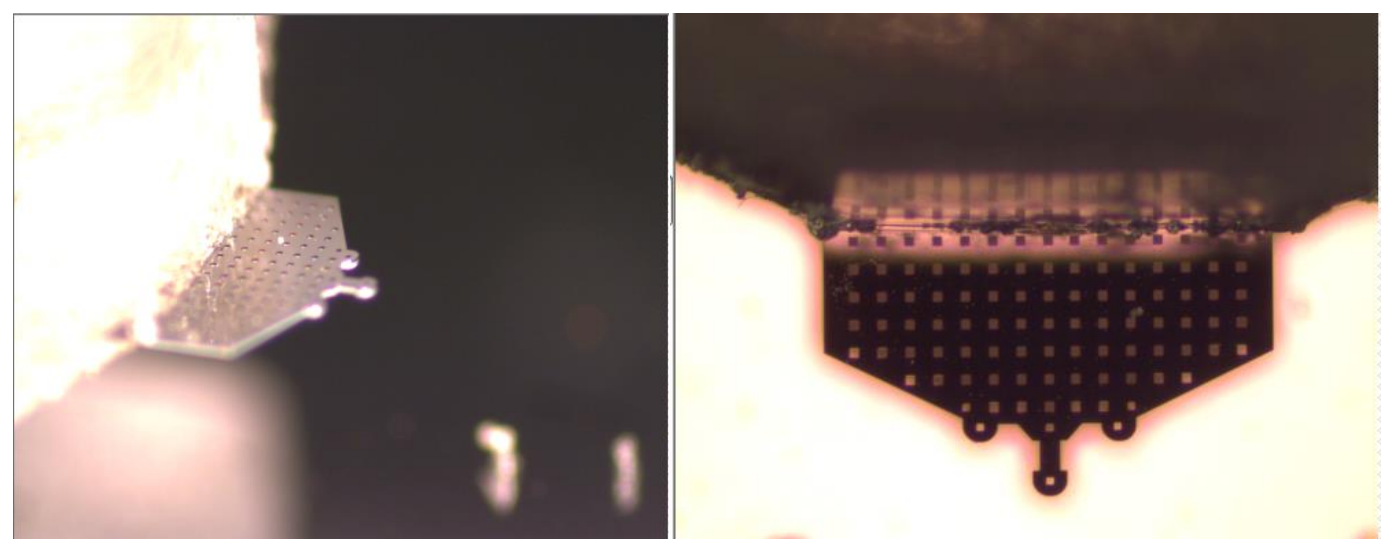

Figure 4.14. Side and top view of single-head microjammer mounted on the end-effector

\subsection{NeXus Calibration and Automation}

After mounting the corresponding microjammer and before starting to assemble microparts, calibration processes for M1 and M2 positioners are needed. For M1 calibration, we implemented both coarse (open-loop) and fine (closed-loop) adjustments. Meanwhile, for M2 calibration, we implemented rotation centering and the 3-point teaching method [66].

\subsubsection{Coarse adjustment}

To calibrate Manipulator 1, the rotation center of the die holder is first moved to the center of the top image as the base frame coordinate $(0,0,0)$. Then, an arbitrary feature at coordinates $\left(\mathrm{x}_{0}, \mathrm{y}_{0}, \theta_{0}\right)$ expressed relative to this center from a certain template, is moved to the center of the top image using inverse kinematics (IK). Assume that the desired orientation of the feature has an angle $\theta_{2}$ relative to the base frame coordinate. The IK operation on the M1 manipulator can be accomplished by rotating the die holder 
to $\theta_{1}$ first, where $\theta_{1}=\theta_{0}+\theta_{2}$. By performing the rotation, the arbitrary feature moves to a new position with the new configuration $\left(\mathrm{x}_{1}, \mathrm{y}_{1}, \theta_{1}\right)$, and then $\mathrm{X}$ and $\mathrm{Y}$ stages of $\mathrm{M} 1$ will move by $-\mathrm{x}_{1}$ and $-\mathrm{y}_{1}$ relative displacements to make the arbitrary point reach the center of the top image with $\theta_{1}$ orientation. These sequences of operations are shown in Figure 4.15. The arbitrary point coordinate is determined by the dimension of both the die holder and die layout of the microstructure.

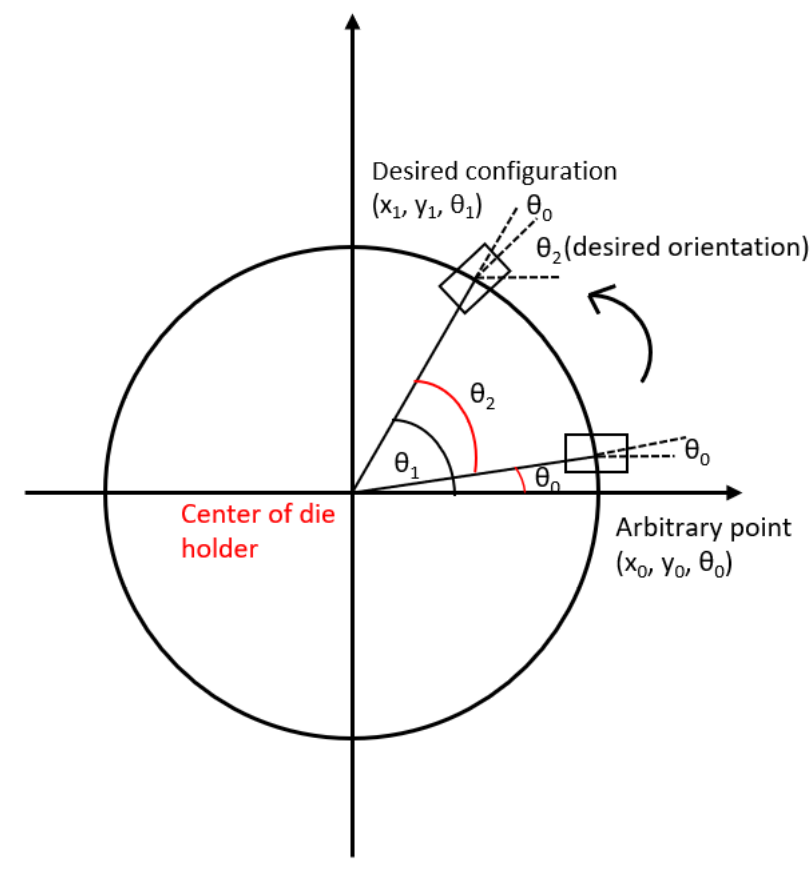

Figure 4.15. Arbitrary point moves to desired configuration

The mathematic relationship between the arbitrary point and its desired configuration can be represented though the following equations:

$$
\begin{gathered}
\theta_{0}=\tan ^{-1} y_{0} / x_{0} \\
\theta_{1}=\theta_{0}+\theta_{2} \\
x_{1}=\sqrt{x_{0}+y_{0}} * \cos \theta_{1}
\end{gathered}
$$




$$
y_{1}=\sqrt{x_{0}+y_{0}} * \sin \theta_{1}
$$

A LabVIEW program was created to accomplish coarse adjustment of M1, with its front-end interface shown in Figure 4.16. By providing the initial feature selection $\mathrm{x}_{0}$, $\mathrm{y}_{0}$, and $\theta_{2}$, then clicking the "Autorun" button, the desired feature will move to the center of the top image with the desired configuration by relative motion of the M1 stages by $\mathrm{x}_{1}$, $\mathrm{y}_{1}$, and $\theta_{1}$.

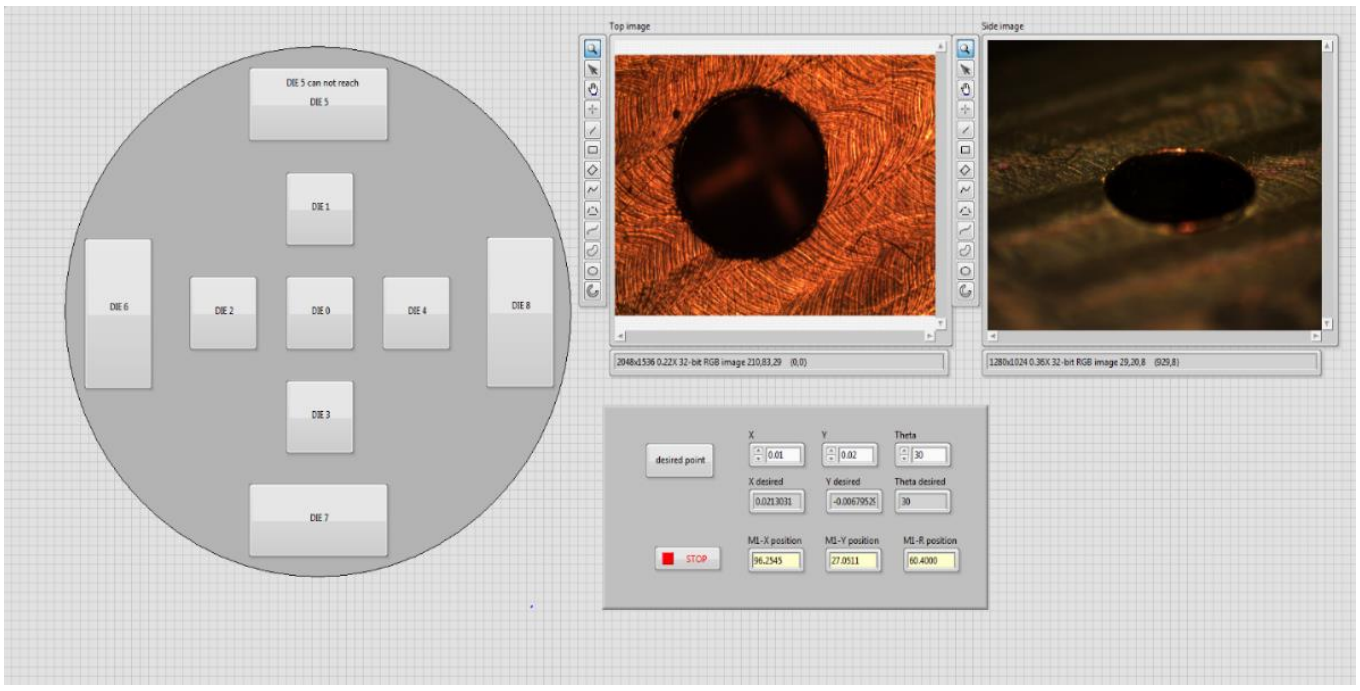

Figure 4.16. Interface for M1 coarse adjustment

To determine the coordinate of $\mathrm{x}_{0}$ and $\mathrm{y}_{0}$ from a template, we need to combine two different coordinate systems, including the die holder coordinate system and the die layout coordinate system. For example, as in Figure 4.17, the center of die holder is the origin coordinate, so each upper left corner of the die chuck has its coordinate referred to the die holder origin. Next, from the die layout, the center of the template can be determined in reference to the origin of each die at the upper left corner (shown in Figure 4.18). After loading the dies on the die holder, the new coordinates of each template 
center, which are listed in Table 4.2, can be determined in reference to the origin of the die holder.

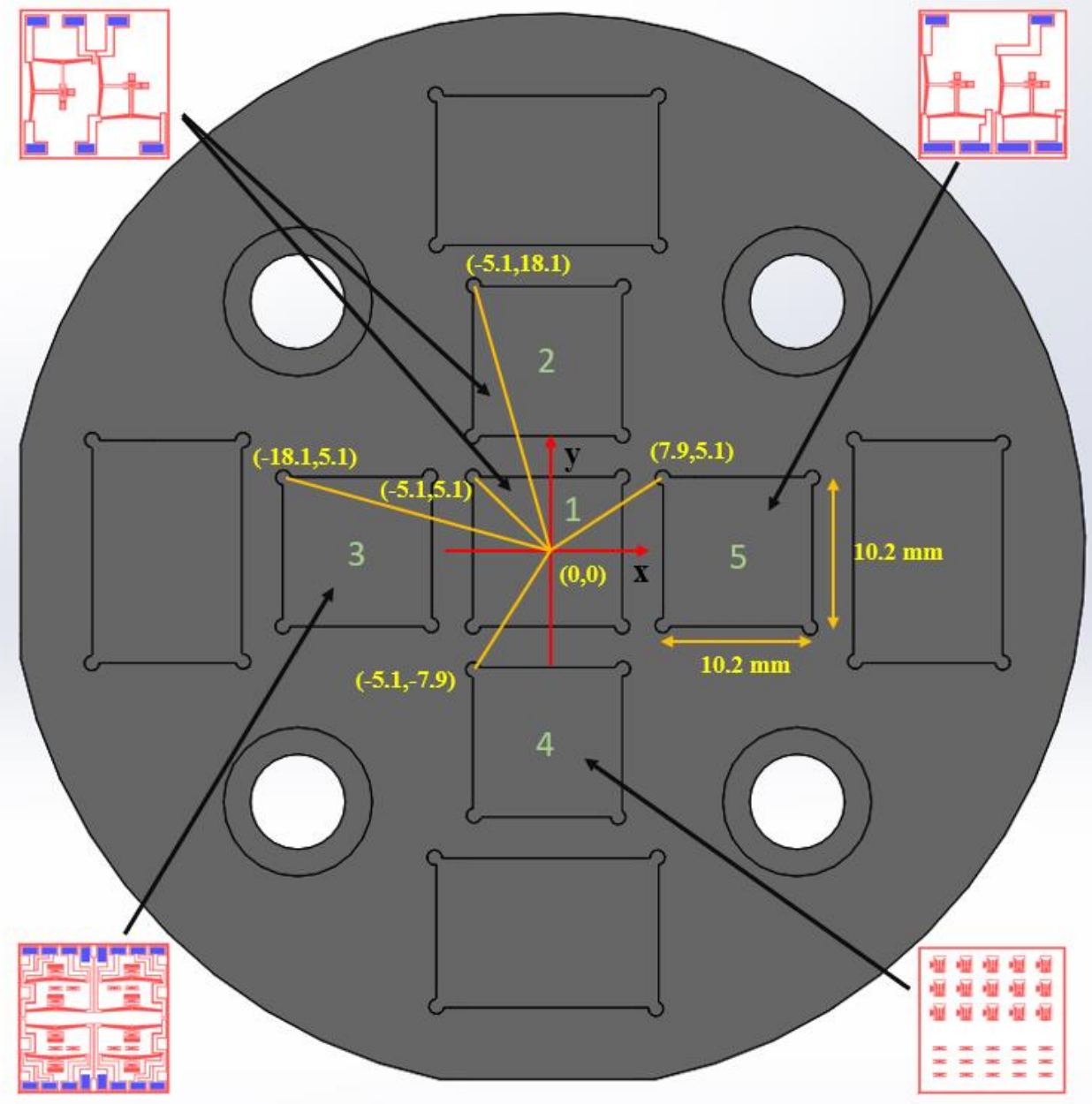

Figure 4.17. Die distribution and coordinates of upper left corner in reference to the origin on the die holder 

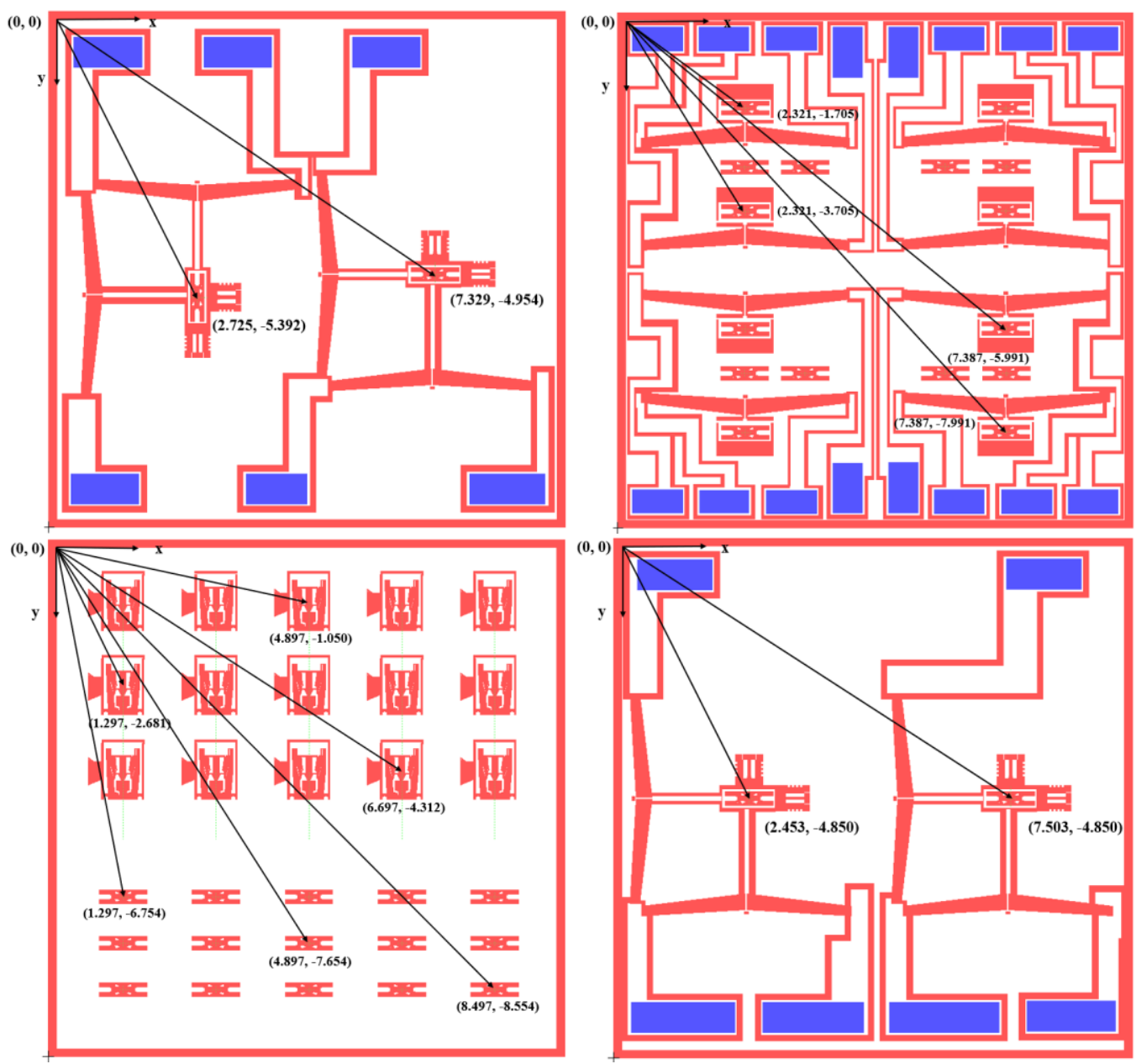

Figure 4.18. Template center coordinates in reference to the origin in the die layout coordinate system 
Table 4.2. The new coordinates of each template center in reference to the die holder origin

\begin{tabular}{|c|c|c|c|c|c|}
\hline & Die 1 & Die 2 & Die 3 & Die 4 & Die 5 \\
\hline $\begin{array}{l}\text { Die holder } \\
\text { origin (left } \\
\text { corner) }(\mathbf{m m})\end{array}$ & $(-5.1,5.1)$ & $(-5.1,18.1)$ & $(-18.1,5.1)$ & $(-5.1,-7.9)$ & $(7.9,5.1)$ \\
\hline $\begin{array}{l}\text { The center of } \\
\text { socket (die } \\
\text { layout) }(\mathrm{mm})\end{array}$ & $\begin{array}{l}(2.725,-5.392) \\
(7.329,-4.954)\end{array}$ & $\begin{array}{l}(2.725,-5.392) \\
(7.329,-4.954)\end{array}$ & $\begin{array}{l}(2.321,-1.705) \\
(2.321,-3.705) \\
(7.387,-5.991) \\
(7.387,-7.991)\end{array}$ & $\begin{array}{l}(1.297,-6.754) \\
(4.897,-7.654) \\
(8.497,-8.554)\end{array}$ & $\begin{array}{l}(2.453,-4.850) \\
(7.503,-4.850)\end{array}$ \\
\hline $\begin{array}{l}\text { Micropart } \\
\text { pickup point } \\
\text { (mm) }\end{array}$ & - & - & - & $\begin{array}{l}(1.297,-2.681) \\
(4.897,-1.050) \\
(6.697,-4.312)\end{array}$ & - \\
\hline $\begin{array}{c}\text { Relative } \\
\text { coordinate } \\
\text { (die holder } \\
\text { origin) }(\mathbf{m m})\end{array}$ & $\begin{array}{l}(-2.375,-0.292) \\
(2.229,-0.146)\end{array}$ & $\begin{array}{l}(-2.375,12.708) \\
(2.229,13.146)\end{array}$ & $\begin{array}{l}(-15.779,3.395) \\
(-15.779,1.395) \\
(-10.713,-0.891) \\
(-10.713,-2.891)\end{array}$ & $\begin{array}{c}(-3.803,-14.654) \\
(-0.203,-15.554) \\
(3.397,-16.454) \\
(-3.803,-10.581) \\
(-0.203,-8.95) \\
(1.597,-12.212)\end{array}$ & $\begin{array}{l}(10.353,0.25) \\
(15.403,0.25)\end{array}$ \\
\hline
\end{tabular}


Table 4.3. 16 testing points moving to desired position with 0 -degree orientation

\begin{tabular}{|c|c|c|c|c|c|c|c|c|c|}
\hline & $\mathbf{X}(\mathbf{m m})$ & $\mathbf{Y}(\mathbf{m m})$ & $\begin{array}{c}\theta \\
(\operatorname{deg})\end{array}$ & X (pixel) & Y (pixel) & $\begin{array}{c}\theta \\
\text { (pixel) }\end{array}$ & $\Delta X($ pixel) & $\Delta Y($ pixel $)$ & $\Delta \theta$ \\
\hline 1 & -2.375 & -0.292 & $\mathbf{0}$ & 952.83 & 747.69 & -1.16 & 71.17 & 20.31 & 1.16 \\
\hline 2 & 2.229 & -0.146 & 0 & 952.66 & 599.63 & -1.18 & 71.34 & 168.37 & 1.18 \\
\hline 3 & -2.375 & 12.708 & 0 & 1052.13 & 874.51 & -0.52 & -28.13 & -106.51 & 0.52 \\
\hline 4 & 2.229 & 13.146 & 0 & 1048.31 & 844.76 & -0.52 & -24.31 & -76.76 & 0.52 \\
\hline 5 & -15.779 & 3.395 & 0 & 1087.79 & 797.39 & -1.47 & -63.79 & -29.39 & 1.47 \\
\hline 6 & -15.779 & 1.395 & 0 & 1081.39 & 804.12 & -1.44 & -57.39 & -36.12 & 1.44 \\
\hline 7 & -10.713 & -0.891 & 0 & 1087.76 & 781.03 & -1.51 & -63.76 & -13.03 & 1.51 \\
\hline 8 & -10.713 & -2.891 & $\mathbf{0}$ & 1083.35 & 783.69 & -1.5 & -59.35 & -15.69 & 1.50 \\
\hline 9 & -3.803 & -14.654 & 0 & 1135.08 & 934.25 & 0.24 & -111.08 & -166.25 & -0.24 \\
\hline 10 & -0.203 & -15.554 & 0 & 1148.29 & 890.23 & 0.76 & -124.29 & -122.23 & -0.76 \\
\hline 11 & 3.397 & -16.454 & 0 & 1162.32 & 850.23 & 0.56 & -138.32 & -82.23 & -0.56 \\
\hline 12 & -3.803 & -10581 & 0 & 1087.6 & 911.4 & -1.2 & -63.6 & -143.4 & 1.2 \\
\hline 13 & -0.203 & -8.95 & 0 & 1068.38 & 857.42 & 0.25 & -44.38 & -89.42 & -0.25 \\
\hline 14 & 1.597 & -12.212 & 0 & 1088.4 & 858.63 & 0.47 & -64.4 & -90.63 & -0.47 \\
\hline 15 & 10.353 & 0.25 & 0 & 1113 & 872.8 & -0.89 & -89 & -104.8 & 0.89 \\
\hline 16 & 15.403 & 0.25 & 0 & 1115.16 & 855.97 & -0.89 & -91.16 & 87.91 & 0.89 \\
\hline
\end{tabular}


Table 4.4. 16 testing points moving to desired position with 30-degree orientation

\begin{tabular}{|c|c|c|c|c|c|c|c|c|c|}
\hline & $X(\mathbf{m m})$ & $Y(m m)$ & $\begin{array}{c}\theta \\
(\mathbf{d e g})\end{array}$ & $\mathbf{X}($ pixel $)$ & Y (pixel) & $\begin{array}{c}\theta \\
(\text { pixel })\end{array}$ & $\Delta$ X(pixel) & $\Delta$ Y(pixel) & $\Delta \theta$ \\
\hline 1 & -2.375 & -0.292 & 30 & 1007.13 & 713.56 & 28.84 & 16.87 & 54.44 & 1.16 \\
\hline 2 & 2.229 & -0.146 & 30 & 937.54 & 583.34 & 28.94 & 86.46 & 184.66 & 1.06 \\
\hline 3 & -2.375 & 12.708 & 30 & 1052.13 & 874.51 & 29.46 & -137.96 & -14.75 & 0.54 \\
\hline 4 & 2.229 & 13.146 & 30 & 1048.31 & 844.76 & 29.4 & -122.15 & 7.22 & 0.6 \\
\hline 5 & -15.779 & 3.395 & 30 & 1087.79 & 797.39 & 28.54 & -122.25 & 70.27 & 1.46 \\
\hline 6 & -15.779 & 1.395 & 30 & 1081.39 & 804.12 & 28.57 & -119 & 68.69 & 1.43 \\
\hline 7 & -10.713 & -0.891 & 30 & 1087.76 & 781.03 & 28.54 & -110.91 & 92.11 & 1.46 \\
\hline 8 & -10.713 & -2.891 & 30 & 1083.35 & 783.69 & 28.57 & -106.09 & 84.9 & 1.43 \\
\hline 9 & -3.803 & -14.654 & 30 & 1135.08 & 934.25 & 30.61 & -233.48 & -10.51 & -0.61 \\
\hline 10 & -0.203 & -15.554 & 30 & 1148.29 & 890.23 & 30.88 & -220.75 & 30.44 & -0.88 \\
\hline 11 & 3.397 & -16.454 & 30 & 1162.32 & 850.23 & 31 & -207.05 & 72.56 & -1 \\
\hline 12 & -3.803 & -10581 & 30 & 1087.6 & 911.4 & 28.79 & -178.79 & -14.76 & 1.21 \\
\hline 13 & -0.203 & -8.95 & 30 & 1068.38 & 857.42 & 30.24 & -140.29 & 18.4 & -0.24 \\
\hline 14 & 1.597 & -12.212 & 30 & 1088.4 & 858.63 & 31 & -154.64 & 23.76 & -1 \\
\hline 15 & 10.353 & 0.25 & 30 & 1113 & 872.8 & 29.07 & -189.8 & 15.31 & 0.93 \\
\hline 16 & 15.403 & 0.25 & 30 & 1115.16 & 855.97 & 29.06 & -186.81 & 38.88 & 0.94 \\
\hline
\end{tabular}

There are 16 template center points in reference to the origin of die holder origin are listed in Table 4.2. In order to determine the accuracy of coarse positioning adjustment, where the center coordinate $(1024,768)$ (in pixel) of top image as the desired position, 16 different template center points have been tested by moving to the desired position with 0 and 30 degrees desired orientations. The results are shown in Table 4.3 and Table 4.4. By comparing the difference of the desired configuration and template center real configurations of 16 testing points, it can be found that with 0 -degree desired orientation, among 16 testing points, the maximum $\Delta \mathrm{X}, \Delta \mathrm{Y}$ (in pixel), and $\Delta \theta$ are - 
$138.32,-166.25$, and 1.51 respectively. Otherwise, with 30-degree desired orientation, the maximum $\Delta \mathrm{X}, \Delta \mathrm{Y}$ (in pixel), and $\Delta \theta$ are $-233.4,184.66$, and 1.46 respectively. According to Figure 4.19, there are three corner coordinates in pixel, as well as length and width of the socket template in micron from the die layout. Therefore, the ratios of distance in pixel and in microns can be expressed (1367-856)/1030 $=0.496$ and (952$756) / 380=0.516$. The average of the ratio is 0.506 , so the maximum distance in $\mathrm{X}$ and $\mathrm{Y}$ with 0 and 30-degree orientation are 273.36 and 328.56 microns, as well as 461.26 and 364.94 microns. The accuracy of the coarse positioning adjustment can be controlled in less than $1 \mathrm{~mm}$.

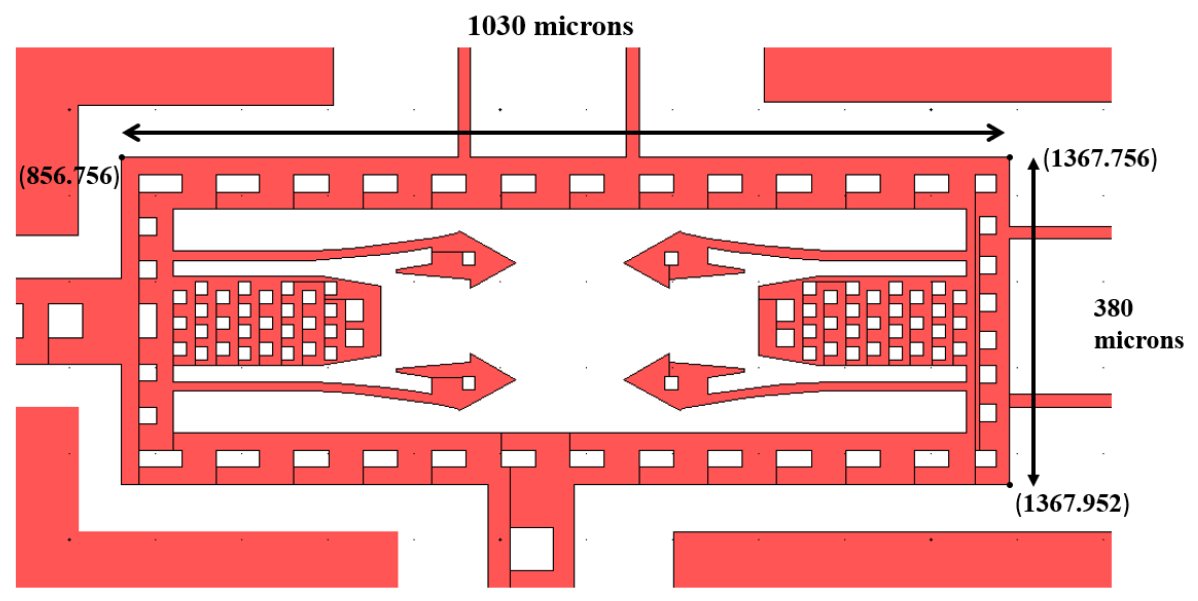

Figure 4.19. Socket template dimensions and three points coordinate in pixel 


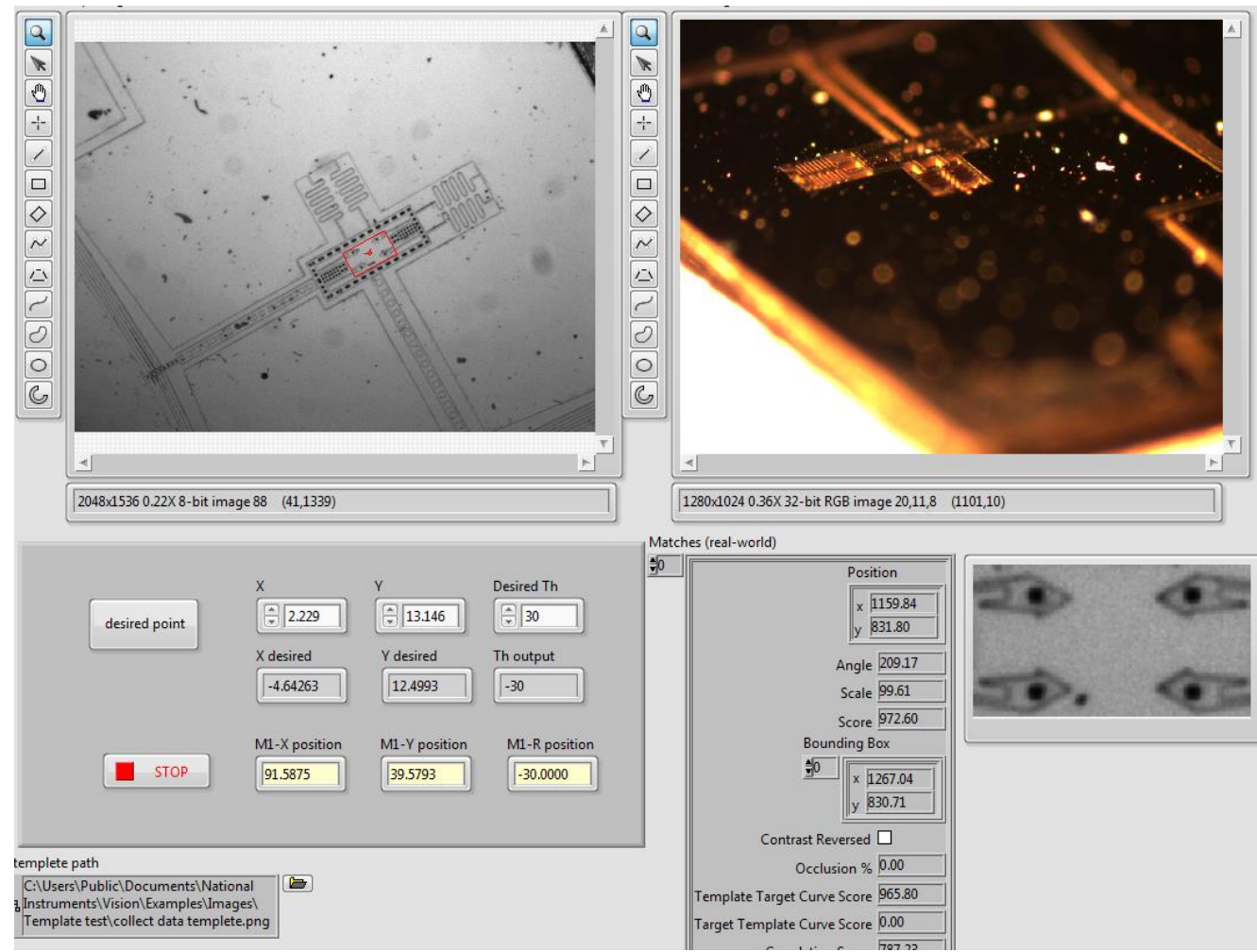

Figure 4.20. Test of desired point movement

\subsubsection{Fine adjustment---visual servoing}

After coarse adjustment of M1, a fine adjustment process to further align desired features to the center of the top microscope image was accomplished using visual servoing. This technique is based on real-time image signals as feedback to actuate multiple stages to adjust the center of a specific feature as a template to move to the desired position in the image. Visual servoing is based on vision feedback for closedcontrol and it has been employed to enhance the accuracy and flexibilities of the robot systems [67-69]. The image Jacobian plays a significant role in visual servoing and can be used to determine the path taken connecting the current image feature template with a desired position in the field of view of the microscope. 
The mathematical equations discussed below detail how to calculate the image Jacobian with respect to differences after stage M1 motion in the configuration of a template center in image pixel. The differences of template center configuration in the top image coordinate in the pixel $\left(\Delta \mathrm{P}_{\mathrm{x}}, \Delta \mathrm{P}_{\mathrm{y}}\right.$, and $\left.\Delta \mathrm{P}_{\theta}\right)$ have the relationship with image Jacobian and the configuration of template center differences $(\Delta \mathrm{X}, \Delta \mathrm{Y}$, and $\Delta \theta)$ shown in equation 4.3.2.1, while the image Jacobian $J^{\prime}$ is expressed in equation 4.3.2.2.

$$
\begin{aligned}
& {\left[\begin{array}{l}
\Delta P_{X} \\
\Delta P_{Y} \\
\Delta P_{\theta}
\end{array}\right]=J^{\prime} *\left[\begin{array}{l}
\Delta X \\
\Delta Y \\
\Delta \theta
\end{array}\right]} \\
& J^{\prime}=\left[\begin{array}{lll}
J_{11}^{\prime} & J_{12}^{\prime} & J_{13}^{\prime} \\
J^{\prime}{ }_{21} & J^{\prime}{ }_{22} & J^{\prime}{ }_{23} \\
J^{\prime}{ }_{31} & J^{\prime}{ }_{32} & J^{\prime}{ }_{33}
\end{array}\right] \\
& \Delta P_{X}=J^{\prime}{ }_{11} * \Delta X+J^{\prime}{ }_{12} * \Delta Y+J^{\prime}{ }_{13} * \Delta \theta \\
& \Delta P_{Y}=J^{\prime}{ }_{21} * \Delta X+J^{\prime}{ }_{22} * \Delta Y+J^{\prime}{ }_{23} * \Delta \theta \\
& \Delta P_{\theta}=J^{\prime}{ }_{31} * \Delta X+J^{\prime}{ }_{32} * \Delta Y+J^{\prime}{ }_{33} * \Delta \theta \\
& {\left[\begin{array}{c}
\Delta P x_{1} \\
\Delta P y_{1} \\
\Delta P_{\theta 1} \\
\Delta P x_{2} \\
\Delta P y_{2} \\
\Delta P_{\theta 2} \\
\Delta P x_{3} \\
\Delta P y_{3} \\
\Delta P_{\theta 3} \\
\vdots
\end{array}\right]=\left[\begin{array}{ccccccccc}
\Delta X_{1} & \Delta Y_{1} & \Delta \theta_{1} & 0 & 0 & 0 & 0 & 0 & 0 \\
0 & 0 & 0 & \Delta X_{1} & \Delta Y_{1} & \Delta \theta_{1} & 0 & 0 & 0 \\
0 & 0 & 0 & 0 & 0 & 0 & \Delta X_{1} & \Delta Y_{1} & \Delta \theta_{1} \\
\Delta X_{2} & \Delta Y_{2} & \Delta \theta_{2} & 0 & 0 & 0 & 0 & 0 & 0 \\
0 & 0 & 0 & \Delta X_{2} & \Delta Y_{2} & \Delta \theta_{2} & 0 & 0 & 0 \\
0 & 0 & 0 & 0 & 0 & 0 & \Delta X_{2} & \Delta Y_{2} & \Delta \theta_{2} \\
\Delta X_{3} & \Delta Y_{3} & \Delta \theta_{3} & 0 & 0 & 0 & 0 & 0 & 0 \\
0 & 0 & 0 & \Delta X_{3} & \Delta Y_{3} & \Delta \theta_{3} & 0 & 0 & 0 \\
0 & 0 & 0 & 0 & 0 & 0 & \Delta X_{3} & \Delta Y_{3} & \Delta \theta_{3} \\
\vdots & \vdots & \vdots & \vdots & \vdots & \vdots & \vdots & \vdots & \vdots
\end{array}\right] *\left[\begin{array}{c}
J^{\prime}{ }_{11} \\
J^{\prime}{ }_{12} \\
J^{\prime}{ }_{13} \\
J^{\prime}{ }_{21} \\
J^{\prime}{ }_{22} \\
J^{\prime}{ }_{23} \\
J^{\prime}{ }_{31} \\
J^{\prime}{ }_{32} \\
J^{\prime}{ }_{33}
\end{array}\right]}
\end{aligned}
$$

Assume: 


$$
Y=\left[\begin{array}{c}
\Delta P x_{1} \\
\Delta P y_{1} \\
\Delta P_{\theta 1} \\
\Delta P x_{2} \\
\Delta P y_{2} \\
\Delta P_{\theta 2} \\
\Delta P x_{3} \\
\Delta P y_{3} \\
\Delta P_{\theta 3} \\
\vdots
\end{array}\right]
$$

$$
A=\left[\begin{array}{ccccccccc}
\Delta X_{1} & \Delta Y_{1} & \Delta \theta_{1} & 0 & 0 & 0 & 0 & 0 & 0 \\
0 & 0 & 0 & \Delta X_{1} & \Delta Y_{1} & \Delta \theta_{1} & 0 & 0 & 0 \\
0 & 0 & 0 & 0 & 0 & 0 & \Delta X_{1} & \Delta Y_{1} & \Delta \theta_{1} \\
\Delta X_{2} & \Delta Y_{2} & \Delta \theta_{2} & 0 & 0 & 0 & 0 & 0 & 0 \\
0 & 0 & 0 & \Delta X_{2} & \Delta Y_{2} & \Delta \theta_{2} & 0 & 0 & 0 \\
0 & 0 & 0 & 0 & 0 & 0 & \Delta X_{2} & \Delta Y_{2} & \Delta \theta_{2} \\
\Delta X_{3} & \Delta Y_{3} & \Delta \theta_{3} & 0 & 0 & 0 & 0 & 0 & 0 \\
0 & 0 & 0 & \Delta X_{3} & \Delta Y_{3} & \Delta \theta_{3} & 0 & 0 & 0 \\
0 & 0 & 0 & 0 & 0 & 0 & \Delta X_{3} & \Delta Y_{3} & \Delta \theta_{3} \\
\vdots & \vdots & \vdots & \vdots & \vdots & \vdots & \vdots & \vdots & \vdots
\end{array}\right]
$$

$$
X=\left[\begin{array}{l}
J_{11}^{\prime} \\
J_{12}^{\prime} \\
J_{13}^{\prime} \\
J_{21}^{\prime} \\
J_{22}^{\prime} \\
J_{23}^{\prime} \\
J^{\prime} 31 \\
J_{32}^{\prime} \\
J^{\prime}{ }_{33}
\end{array}\right]
$$

So:

$$
Y=A * X
$$

The $\mathrm{X}$ can be transformed to

$$
X=\left[A^{T} * A\right]^{-1} * A^{T} * Y
$$




$$
\left[\begin{array}{c}
X_{\text {new }}-X_{c} \\
Y_{\text {new }}-Y_{c} \\
\theta_{\text {new }}-\theta_{c}
\end{array}\right]=\Delta s * J^{-1} *\left[\begin{array}{l}
P_{X_{d}}-P_{X_{c}} \\
P_{Y_{d}}-P_{Y_{c}} \\
P_{\theta_{d}}-P_{\theta_{c}}
\end{array}\right]
$$

After obtaining the image Jacobian from experiments, the template motion can be moved to the desired position with the desired orientation according to equation (4.3.2.10). The constant $\Delta \mathrm{s}$ can determine the step size of movement of multiple stages. A LabVIEW program was created to implement the visual servoing algorithm, including three sub-interfaces, manual control, image Jacobian generation, and automated control shown in Figure 4.21.
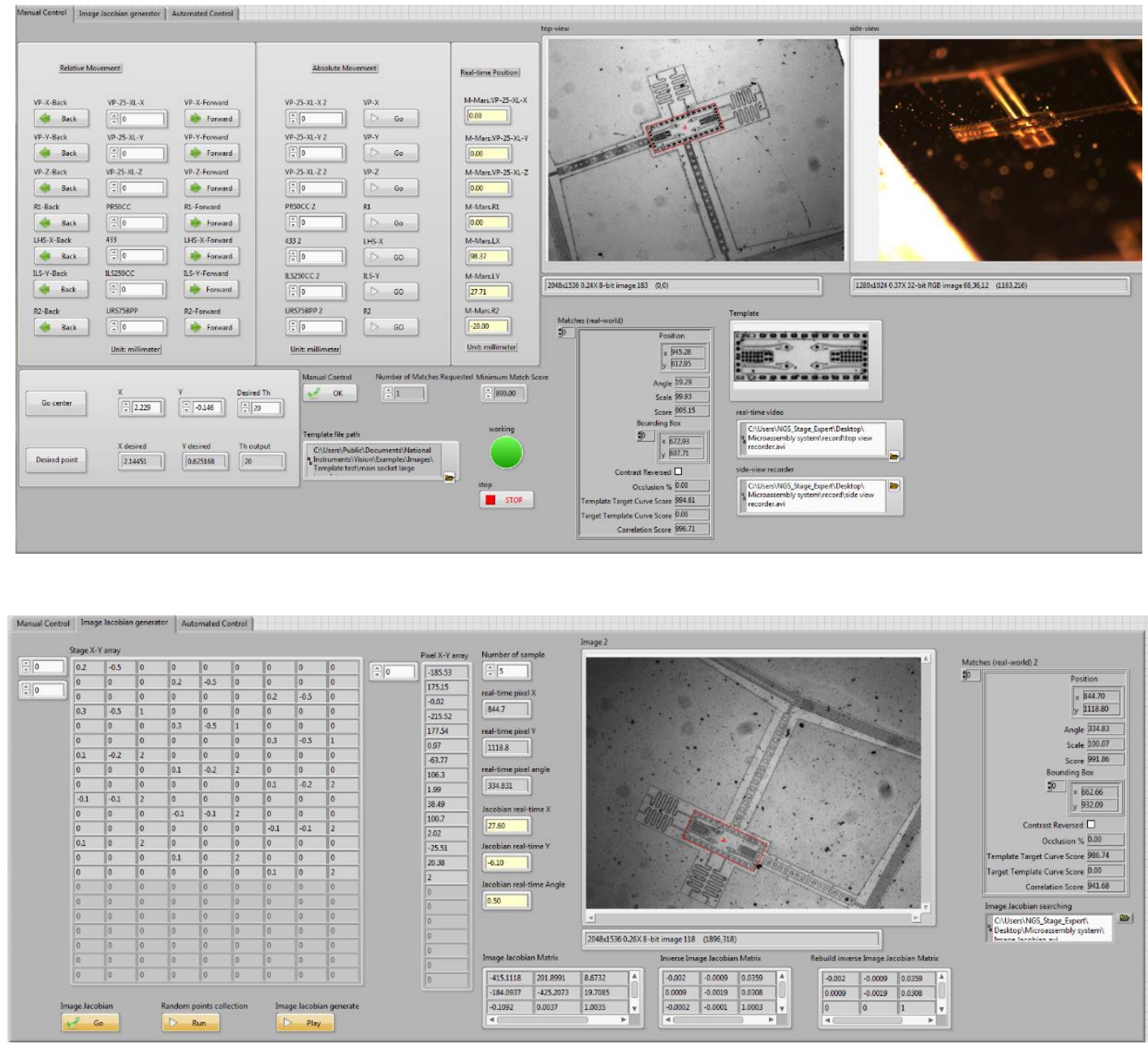


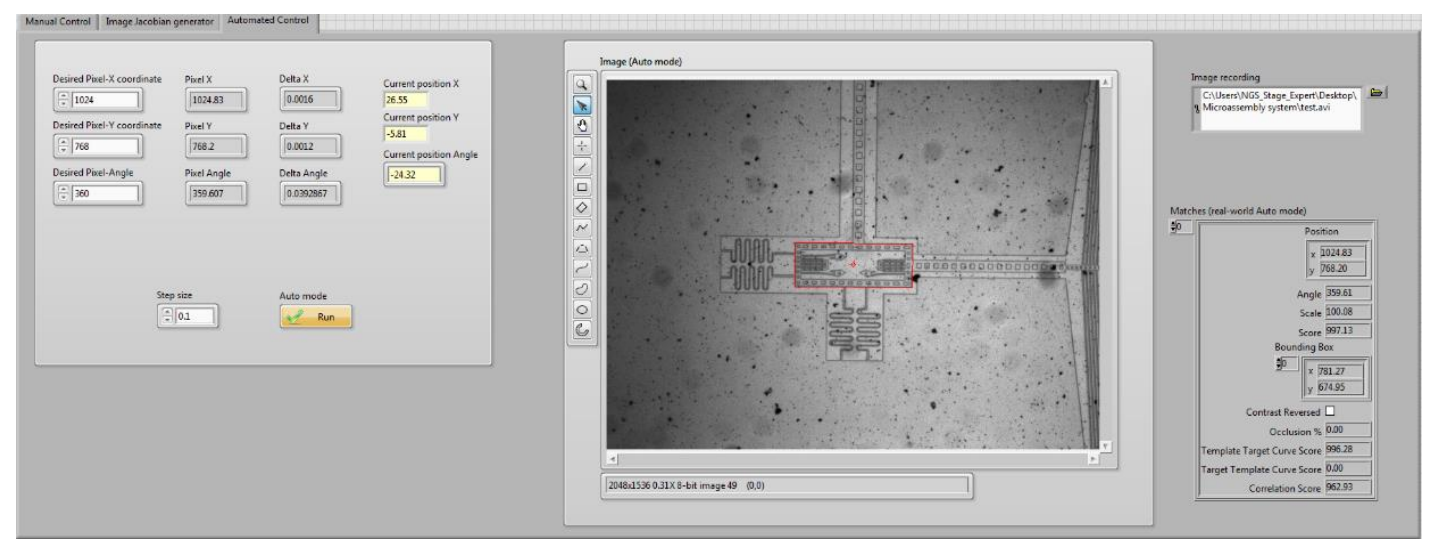

Figure 4.21. Three interfaces for semi-automation calibration of M1

Finally, as a result of the combination of coarse and fine adjustments for M1, any microstructure can be moved to the desired configuration in the center of the image field of view, thus providing a reliable location for subsequent assembly of a micropart onto the template.

\subsubsection{Rotation centering}

For manipulator 2 calibration, the position of the microjammer tip needs to be adjusted such that a M2 stage rotation will generate a motion around the jammer tip through a process called "rotation centering" depicted in Figure 4.22).
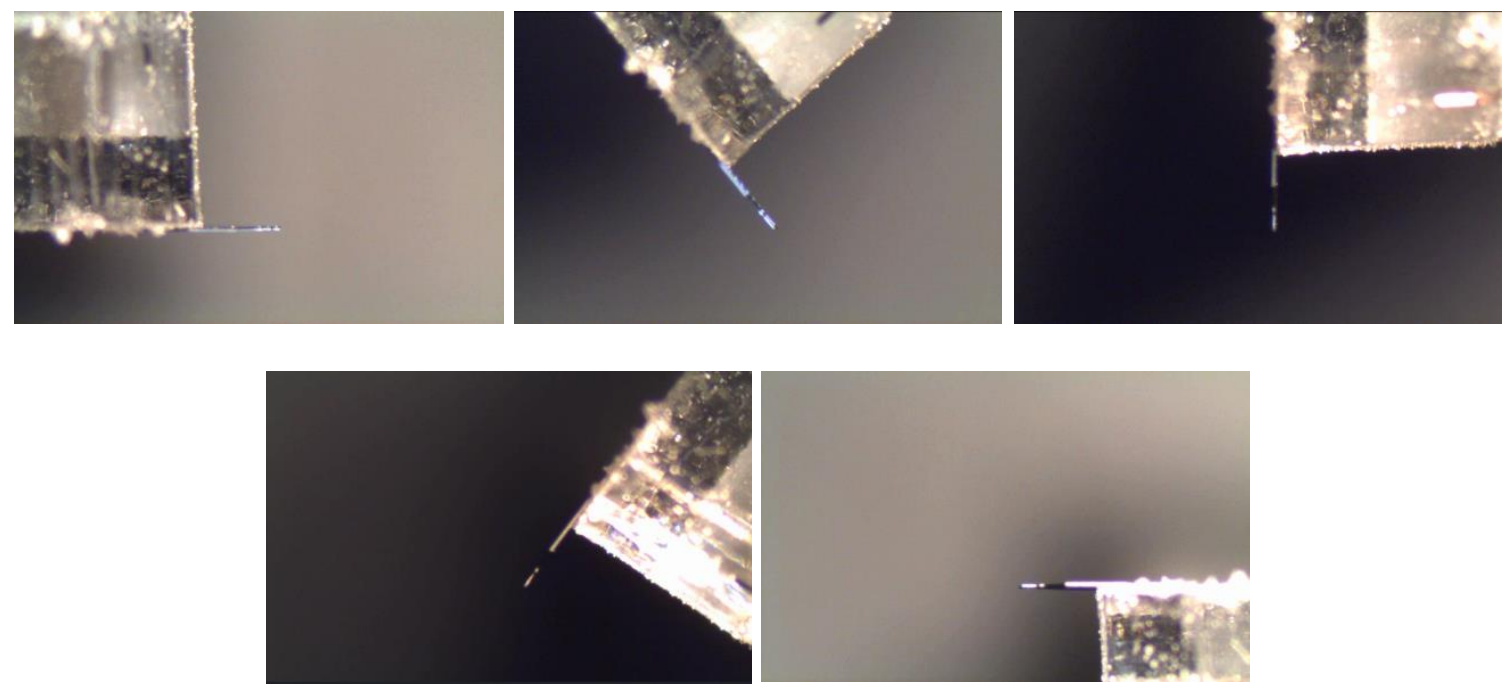
Figure 4.22. Rotation centering for the microjammer tip

After mounting the microjammer on the end-effector, the calibration of the microjammer will be operated by the X-Y manual translation stage (shown in Figure 4.23) to move the tip of the microjammer to the center of the M2 rotation stage. The merits of rotation centering are that it keeps the picked micropart staying in the field of view in the image, and it enables the configuration of the micropart can be easier to determine by some constant parameters.

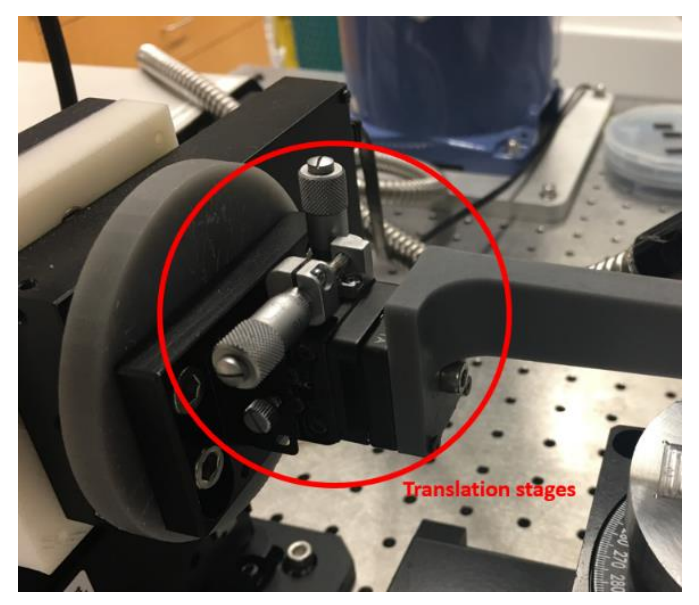

Figure 4.23. Manual translation stage for rotation centering

\subsubsection{3-point teaching method}

After calibration of the microjammer, a further calibration has been done with a 3point teaching method, which is "a simple, but very effective calibration scheme based on linear interpolation of a set of taught fiducials"[66]. This method is used to calibrate M1 and M2 by controlling the tip of the microjammer to attach 3 arbitrary points of die on M2. When the tip attaches the die, its M1 encoder coordinate of M1 will be recorded. Based on three different points of encoder data, any points from die coordinates can be 
calculated according to the transformation of the encoder coordinate by the following equations[66]:

$$
\begin{gathered}
E=E_{1}+\left(E_{2}-E_{1}\right)\left(\frac{a-a_{1}}{a_{2}-a_{1}}\right)+\left(E_{3}-\hat{\mathrm{E}}\right)\left(\frac{b-b_{1}}{b_{3}-b_{1}}\right) \\
\hat{\mathrm{E}}=E+\left(E_{2}-E_{1}\right)\left(\frac{a_{3}-a}{a_{2}-a}\right)
\end{gathered}
$$

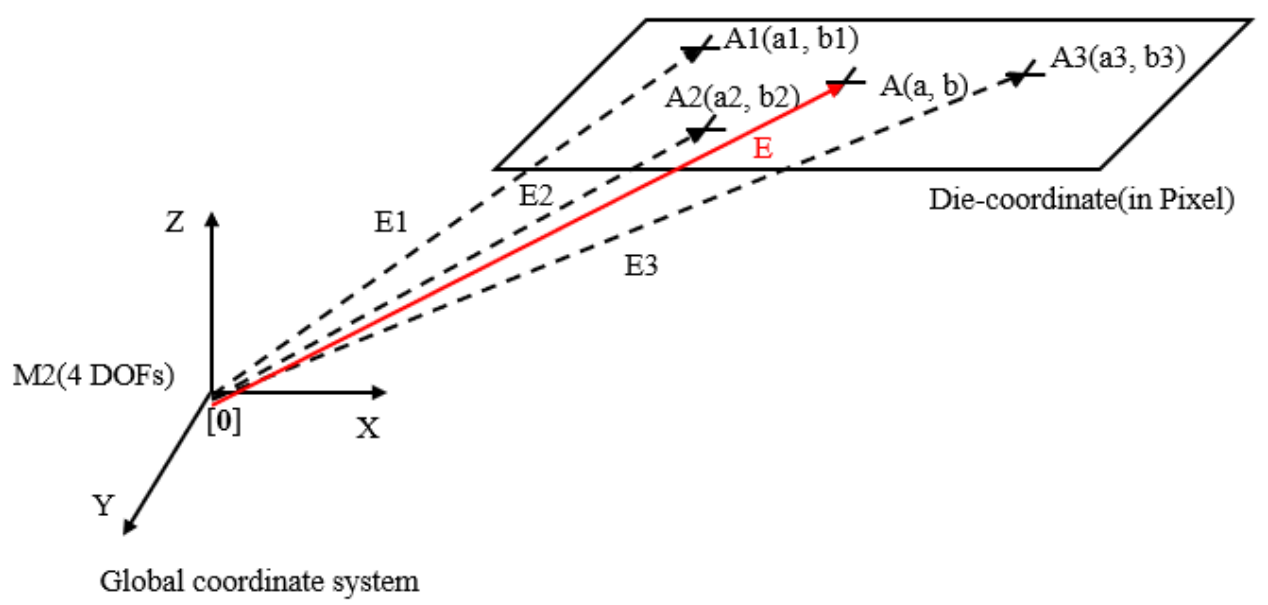

Figure 4.24. 3-point teaching method[66]

1) Select three random points $A 1, A 2$, and A3 on the MEMS die, with $\left(a_{1}, b_{1}\right),\left(a_{2}\right.$, $\left.b_{2}\right)$ and $\left(a_{3}, b_{3}\right)$ in die coordinate respectively. Those coordinate values can be acquired in pixels from the top imaging system, or obtained from die layout coordinates, the fabrication tolerances can be ignored.

2) A is any desired point with die coordinates (a, b). Later, it can become the mark of an assembly site. 
3) Control manipulation 2 to make the microjammer tip to touch A1, A2, and A3 and obtain the parameters of each point as encoder vectors E1, E2, and E3. Since M2 has 4 DOFS, these should be 4-dimensional vectors [Encoder X; Encoder Y;

Encoder Z; Encoder $\theta]$.

4) $\mathrm{E}$ is the encoder vector when the microjammer tip is touching to $\mathrm{P}[66]$.

Based on the 3-point teaching method above, a single-head microjammer is used to calibrate M2 as shown in Figure 4.21. Recorded E1, E2, and E3 encoder coordinates during this process are summarized in Table 4.5. According to the equation 4.3.4.1, the center of Zyvex socket configuration $\mathrm{E}=(-3.35,-5.485,8.91,0)$, where $A_{1}(635,728), A_{2}(1382,728), A_{3}(635,1004)$, and $A(1008,866)$.

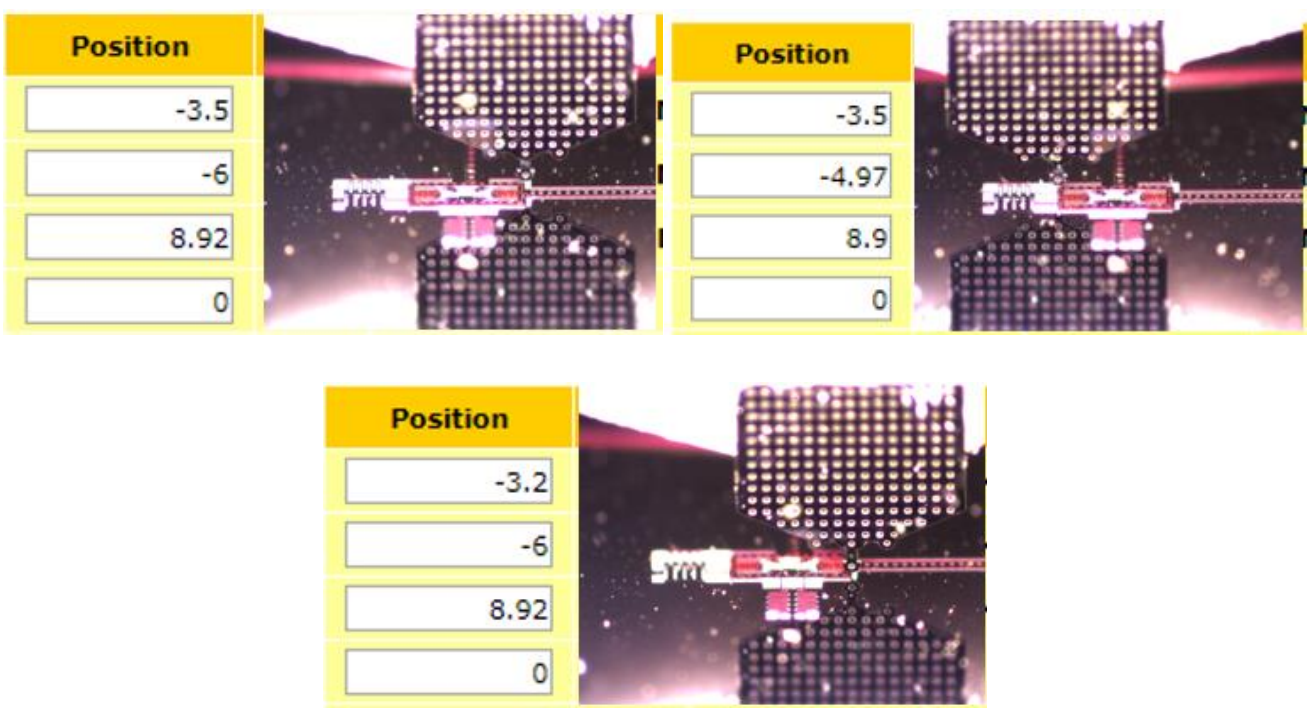




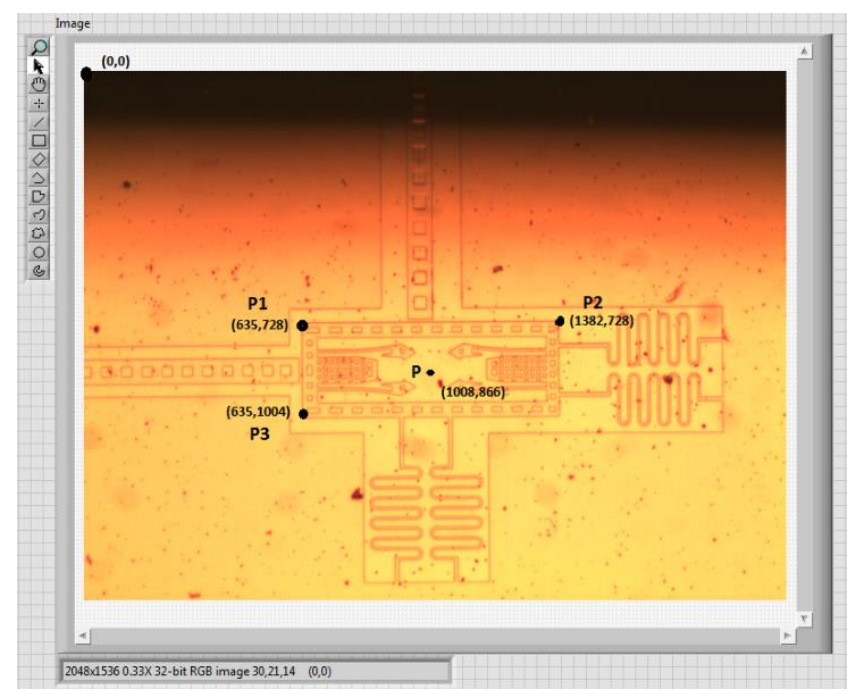

Figure 4.25. Single-head microjammer to calibrate M1

Table 4.5. Encoder X, Y, Z, $\theta$ of M2

\begin{tabular}{|c|c|c|c|c|}
\hline & Encoder X & Encoder Y & Encoder Z & Encoder $\boldsymbol{\theta}$ \\
\hline E1 & -3.5 & -6 & 8.92 & 0 \\
\hline E2 & -3.5 & -4.97 & 8.9 & 0 \\
\hline E3 & -3.2 & -6 & 8.92 & 0 \\
\hline $\mathbf{E}$ & -3.35 & -5.485 & 8.91 & 0 \\
\hline
\end{tabular}

\subsubsection{Pick and Place}

After calibration of M1 and M2, the serial microassembly process via automation can be used to assemble desired microparts for the AFAM and SAFAM, using the basic Zyvex snap-fastener microstructure.

\subsubsection{Jammer part assembly testing}

Using a single-head microjammer, single Zyvex snap-fastener microstructures were successfully assembled to the Zyvex socket by pick-rotate-place steps as shown in 
Figure 4.26, which means that the accuracy of the NeXus microassembly system is acceptable
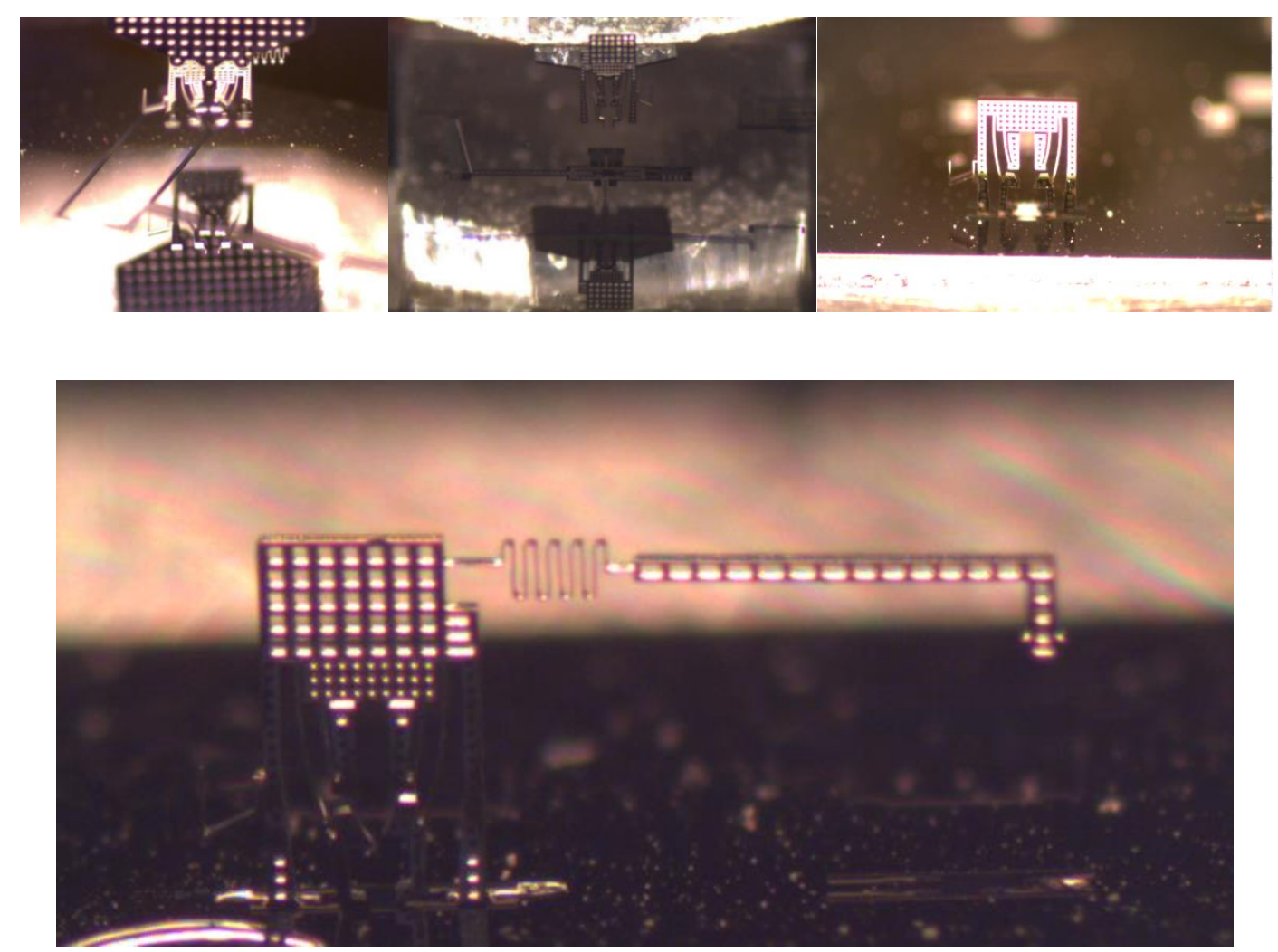

Figure 4.26. Pick-rotate-place for part assembly testing and assembled AFAM

\subsubsection{2 sAFAM assembly and bonding}

Additional challenges were encountered in the assembly sAFAM because the large size of the unibody sAFAM $(10 \mathrm{~mm} \times 10 \mathrm{~mm} \times 1.5 \mathrm{~mm})$ is outside the field of view in the top imaging system. Only one of the jammer or Zyvex microstructures appears in the image. So, it is harder to mount a dual-head microjammer and align the jammer with the snap-fastener part. The solution was to move the top image in X-axis and with the assistance from the side imaging system. With this method, the sAFAM was assembled successfully using the manual control as shown in Figure 4.27. After assembly, it is 
necessary to bond the SAFAM to fix it on the substrate. To this end, we mounted a fiber on the end-effector and used it to transfer a small amount of UV light epoxy adhesive (Thorlabs Norland optical adhesive) onto the arm assembly legs, which will become solid after curing. By repeating wicking and curing steps to all 4 stands of the sAFAM arm, we completed the assembly and bonding of the microrobot. During the bonding process, M1 was adjusted to reach the proper angle for fiber attachment in the desired orientation, while the side imaging system provided a better image for the bonding process.
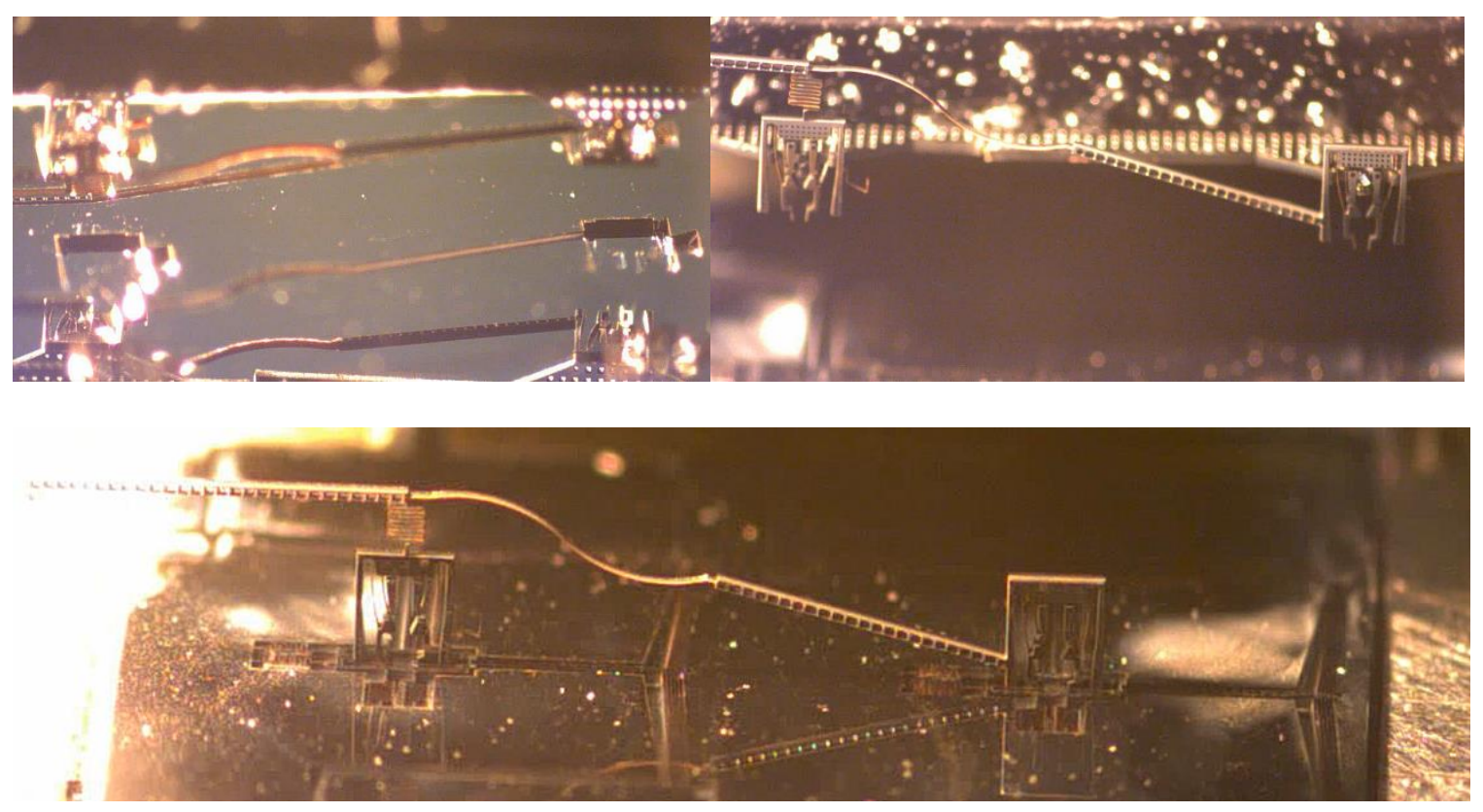

Figure 4.27. Picking-up, rotating, and placing for sAFAM assembly

After assembling the sAFAM, two manual probe tips were added onto the NeXus microassembly system to apply actuation voltages while we measured the displacements of the cantilever tip of the sAFAM. Applied voltages ranging from $20 \mathrm{~V}$ to $35 \mathrm{~V}$ were applied onto the Chevron actuators implementing the X-Y actuator banks of the sAFAM. The comparison of sAFAM experiment and simulation results using Ansys ${ }^{\circledR}$ are 
expressed in Table 4.4. Results show that experimental displacements of the tip in Z-axis is close to those in simulation results, especially with higher voltages applied.

Table 4.6. Comparison of sAFAM experiment and simulation results

\begin{tabular}{|c|c|c|c|c|c|}
\hline \multirow{7}{*}{} & Applied Voltage & $20 \mathrm{~V}$ & $25 \mathrm{~V}$ & $30 \mathrm{~V}$ & $35 \mathrm{~V}$ \\
\cline { 2 - 6 } & $\begin{array}{c}\text { Displacement of Chevron } \\
\text { Actuator }(\mu \mathrm{m})\end{array}$ & 2 & 11 & 17.5 & 22 \\
\hline \multirow{3}{*}{$\begin{array}{c}\text { Experiment } \\
\text { results }(\mu \mathrm{m})\end{array}$} & $\mathrm{A}(-\mathrm{Z})$ & 31 & 46 & 63 & 79 \\
\cline { 2 - 6 } & $\mathrm{B}(-\mathrm{Y})$ & 22 & 43 & 70 & 93 \\
\cline { 2 - 6 } & $\mathrm{C}(+\mathrm{Z})$ & 8.3 & 46 & 73.3 & 92 \\
\hline \multirow{3}{*}{$\begin{array}{c}\text { Simulation } \\
\text { results }(\mu \mathrm{m})\end{array}$} & $\mathrm{D}(+\mathrm{Y})$ & 1.1 & 6 & 10 & 12.5 \\
\cline { 2 - 6 } & $\mathrm{A}(-\mathrm{Z})$ & 8.4 & 46 & 73.5 & 93 \\
\cline { 2 - 6 } & $\mathrm{B}(-\mathrm{Y})$ & 3.1 & 17.2 & 27.4 & 34.4 \\
\hline
\end{tabular}

The workspace of sAFAM can reach an estimated area of $22 \mu \mathrm{m} * 47 \mu \mathrm{m} * 185 \mu \mathrm{m}$, while the simulated workspace was $21 \mu \mathrm{m} * 44 \mu \mathrm{m} * 172 \mu \mathrm{m}$. In Pitch motion $(+/-\mathrm{Z})$, when the applied voltage increases, the results from simulation and experiments are fairly close. 


\section{CHAPTER 5}

\section{FLEXIBLE SKIN SENSOR FABRICATION AND EVALUATION}

During this research, I conducted additional studies on the fabrication of flexible skin sensors. During the last few years, advances have been made in fabrication, packaging, and interconnections of tactile-sensitive skins to robots [22-25]. In our

previous work, a sensor with Interdigitated Elements (IDE) structures has been patterned onto flexible Kapton ${ }^{\circledR}$ substrates, and coated with a polymer piezo-resisting material, Poly (3,4-ethylene dioxythiophene)-poly (styrene sulfonate) or PEDOT: PSS, by Electro Hydro Dynamic (EHD) printing. The resulting sensor skins have strain gauges arrays with a high gauge factor (GF) [26], that can transfer applied pressure to strain dependent upon the resistance change property of PEDOT: PSS [13, 27, 28].

To replace EHD printing, a novel method, which is called "wet lift-off photolithographic technique" was developed to pattern pressure single sensors or sensor arrays. This technique is based on $0.8 \%$ PEDOT: PSS colloid in $\mathrm{H} 2 \mathrm{O}$ with varying ratios of Methanol to obtain desirable wetting and uniform spinning.

\subsection{Fabrication of electrodes on flexible Kapton}

High density pressure sensitive arrays with PEDOT: PSS piezoresistive materials on Kapton ${ }^{\circledR}$ substrates have been prototyped using well-known cleanroom techniques that were adapted for the choice of skin materials. Figure 5.1 outlines the fabrication process leading to sensors patterned over $300 \mathrm{~nm}$ thick gold traces on $50 \mu \mathrm{m}$ thick Kapton 
sheet. This process uses a carrier wafer to support the Kapton through processing. Otherwise, this is a standard process for liftoff and can be applied to most deposited materials on various substrates. Optimally, a polyamide precursor is spun onto a wafer avoiding the backing of pre-purchased, extruded Kapton. This provides higher quality and consistent micro-patterned structures. In previous work to optimize the process, ebeam and sputter deposited thin films were inspected under SEM and optical profilometry, and special attention was paid to avoid cracks. Sputter deposited Au thin films appeared to adhere better than E-beam films in our applications. Au was sputtered directly onto Kapton and our tests suggest that under normal conditions, the films would not fracture when the substrate was being bent to a $5 \mathrm{~mm}$ radius. Integrity of the traces undergoing cyclic testing has yet to be studied. The detailed flexible skin sensor fabrication steps can be found in APPENDIX B.

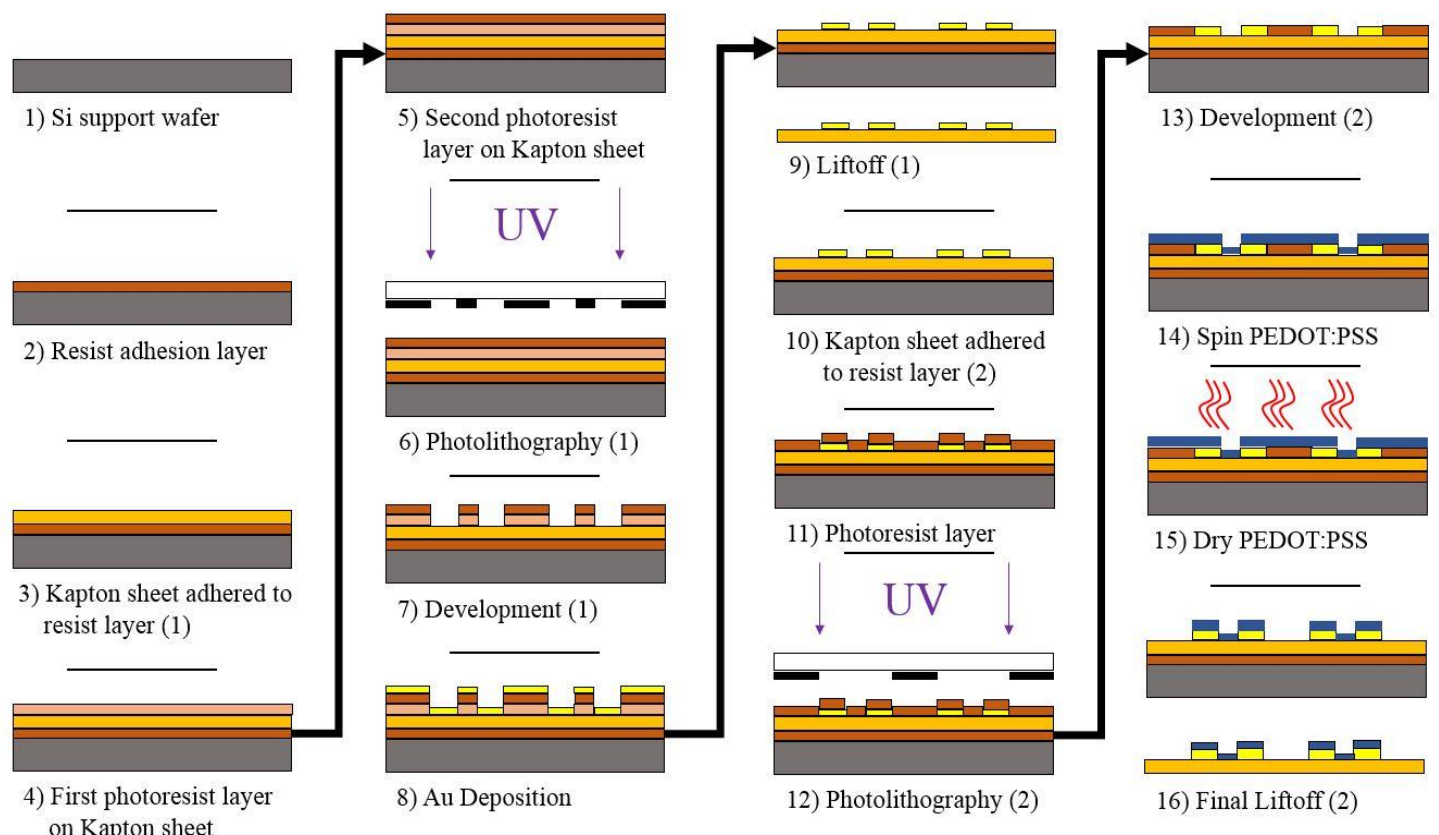

Figure 5.1. Skin sensor fabrication steps [13] 


\subsection{Development of PEDOT:PSS solutions for spin coating}

In this chapter, we report a novel deposition and patterning process for PEDOT:PSS via spin coating and wet lithography employing a simple process that would allow for more cost effective mass production. Traditionally, lift-off is difficult when working with organic materials due to issues with adhesion during pattern definition. Processing often employ methods such as masking with Parylene C, dry development and/or etching. Our work has shown that dried PEDOT:PSS adheres well to cleaned Kapton substrates, and allows for wet lift-off processing and for subsequently defining PEDOT:PSS thin films. Solutions containing PEDOT:PSS for our applications are critical for the piezoresistive nature of the strain gauges. PEDOT:PSS materials available from distributors are often not suitable alone for their processing in microfabrication. Thus, one must add compounds to such materials to increase their wettability on the application surface, and also tailor those compounds for the method of deposition. More specifically, in our application, we sought mixtures that support their uniform coating when spin coated on a wafer supporting Kapton films.

During this research, different types of PEDOT solutions were formulated using PEDOT:PSS in $\mathrm{H} 2 \mathrm{O}$ as a functional material. These mixtures were tested for spin quality and wettability of our mixtures. First, PEDOT:PSS polymer solution was mixed in varying volume ratios (from 1:1-1:4, PEDOT:PSS : Methanol) to obtain desired viscosity and surface tension. Methanol was selected due to its low surface tension and low boiling point. A mixture of N-Methyl-2-pyrrolidone (NMP) and PEDOT:PSS were studied, and while it performed to our expectations, it induced aggregation and was ultimately discarded. All ratios were spun onto RIE cleaned Kapton, and all samples exhibited 
sufficiently uniform coatings, while all conductivity as measured by a standard digital multi-meter was probed by hand.

After spinning PEDOT:PSS based solutions onto interdigitated structures, it is necessary to encase the sensor in a material that prevents permeation of moisture or adsorption of other contaminants into the film. Optimally, this is done with a Polyamide precursor, but in our works, we encapsulate the sensors with Kapton tape. After the wet lift-off process to define the PEDOT:PSS is completed, sensors are diced and the substrates are left to dry in a convection oven at 100 degrees Celsius for 15 minutes to remove excess moisture. After this step is complete, sensors are removed one at a time and covered with Kapton tape to protect the strain gauges, as shown in Figure 5.2.
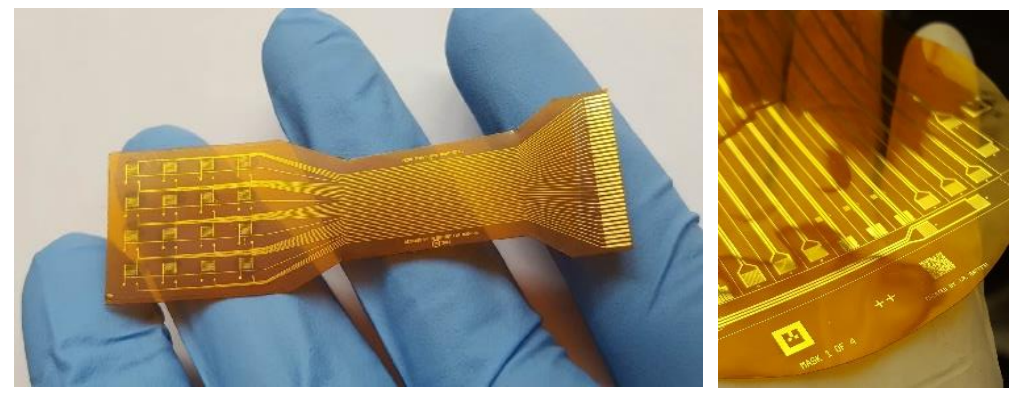

Figure 5.2. Examples of IDE structure made of gold on Kapton Sheet [13]

To evaluate the flexible skin sensor, measurements of sheet resistance and resistivity measurements of PEDOT:PSS thin films coated on Kapton were carried out as summarized in Table 5.1. Two PEDOT:PSS solution samples (1:1 and 1:4 ratios of (PEDOT:PSS): Methanol) were spun onto test Kapton Substrates at 2,500 rpm and measured at 5 points using a Lucas Labs 4-Point probe station [13] (shown in Figure 5.3). 


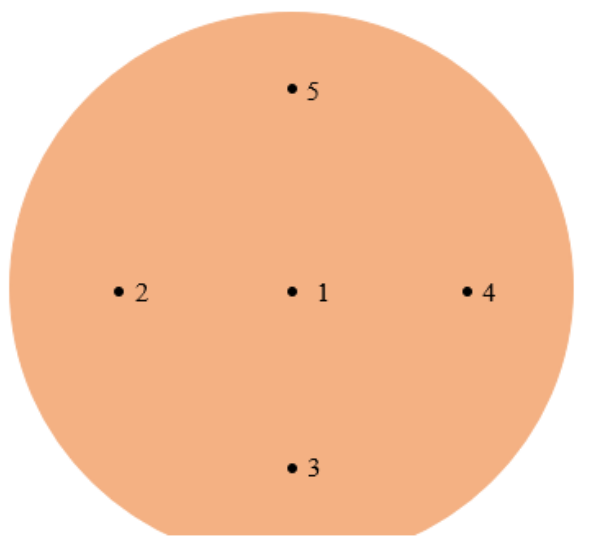

Figure 5.3. 5-point measurements on Kapton sheet by 4-point probe station

Table 5.1. Measurements of sheet resistance and resistivity for PEDOT:PSS thin films coated on Kapton.

\begin{tabular}{|c|c|c|c|}
\hline \multicolumn{2}{|c|}{$1: 1(8 \mathrm{ml}: 8 \mathrm{ml})$} & \multicolumn{2}{c|}{$1: 4(3 \mathrm{ml}: 12 \mathrm{ml})$} \\
\hline Res(ohms/square) & Res(ohms-cm) & Res(ohms/square) & Res(ohms-cm) \\
\hline 1.795 .807922 & 0.007958 & 1.1073 .573608 & 0.010736 \\
\hline 2.949 .151672 & 0.009492 & 2.2001 .454834 & 0.020015 \\
\hline 3.943 .163513 & 0.009432 & 3.1340 .098877 & 0.013401 \\
\hline 4.927 .551453 & 0.009276 & 4.1301 .431763 & 0.013014 \\
\hline 5.977 .226827 & 0.009772 & 5.1912 .351074 & 0.019124 \\
\hline Avg: 918.580261 & 0.009186 & Avg: 1525.781982 & 0.015258 \\
\hline
\end{tabular}

From Table 5.1, we can preliminarily conclude that the sheet resistance and resistivity of PEDOT:PSS thin films increases by when smaller amounts of PEDOT:PSS are present in the mixture solution.

After testing the pure Kapton sheet with two different PEDOT:PSS solutions, the patterned skin sensor structures with different PEDOT:PSS solution were also tested 
under different process parameter, such as coating spin speed, concentration of PEDOT:PSS, and ratio of PEDOT and methanol. Table 5.2 summarizes process conditions from 8 different batches which consists of two spin speeds: $750 \mathrm{rpm}$ and 1500 rpm; two PEDOT:PSS concentrations: $0.8 \%$ and 4\%; and two ratios of PEDOT:PSS and methanol solution: $1: 1(2.5 \mathrm{ml}: 2.5 \mathrm{ml})$ and $2: 1(4 \mathrm{ml}: 2 \mathrm{ml})$.

Table 5.2. 8 batches of different parameters for PEDOT:PSS tests

\begin{tabular}{|c|c|c|c|c|c|c|c|c|}
\hline & 1 & 2 & 3 & 4 & 5 & 6 & 7 & 8 \\
\hline Speed & $750 \mathrm{rpm}$ & $750 \mathrm{rpm}$ & $750 \mathrm{rpm}$ & $750 \mathrm{rpm}$ & $1500 \mathrm{rpm}$ & $1500 \mathrm{rpm}$ & $1500 \mathrm{rpm}$ & $1500 \mathrm{rpm}$ \\
\hline Con & $0.8 \%$ & $0.8 \%$ & $4 \%$ & $4 \%$ & $0.8 \%$ & $0.8 \%$ & $4 \%$ & $4 \%$ \\
\hline ratio & $1: 1$ & $2: 1$ & $1: 1$ & $2: 1$ & $1: 1$ & $2: 1$ & $1: 1$ & $2: 1$ \\
\hline
\end{tabular}

After fabricating skin sensors with 8 batches of recipes, the resistance of each skin sensor were measured on the heads and tails of the sensors by probe station and are shown in Figure 5.5 and all the measured results are shown in Figure 5.4. The resistances of all skin sensors on the heads are between 6 and $11 \mathrm{ohms}$, and on the tails are in range of 22 up to 28 ohms. The main difference is due to the long traces from head to tails. Moreover, during the PEDOT:PSS releasing step, we found when using higher concentration PEDOT:PSS solution, no matter what spin speed and ratio was observed with a methanol mixture solution, there existed some PEDOT:PSS residues left on the surface. Therefore, batch 3, 4, 7, and 8 were not recommended to be applied. Among batch 1,2,5, and 6, the batch 1 is the best recipe for coating PEDOT:PSS on the skin sensor. We used a testing motherboard to test batch 1 skin sensors, and the drifts were clearer when they were applied by external force. 


\begin{tabular}{|c|c|c|c|c|c|c|c|c|c|}
\hline \multirow[b]{5}{*}{ Sensor no. } & & \multicolumn{2}{|l|}{ Batch 1} & \multicolumn{2}{|l|}{ Batch 2} & \multicolumn{2}{|l|}{ Batch 3} & \multicolumn{2}{|l|}{ Batch 4} \\
\hline & & Speed (rpm) & 750 & Speed (rpm) & 750 & Speed (rpm) & 750 & peed (rpm) & 750 \\
\hline & & Ratio (PEDOT/Methanol) & 1 & Ratio (PEDOT/Methanol) & 2 & Ratio (PEDOT/Methanol) & Ra & latio (PEDOT/Methanol) & 2 \\
\hline & & Concentration $\%$ & 0.8 & Concentration \% & 0.8 & Concentration $\%$ & co & Concentration \% & 4 \\
\hline & & Min Res. & Max Res. & Min Res. & Max Res. & Min Res. & Max Res. & Min Res. & Max Res. \\
\hline \multirow[b]{2}{*}{1} & Head & 11.11 & 11.51 & 6.437 & 6.457 & 10.001 & 10.232 & 6.001 & 6.023 \\
\hline & Tails & 23.738 & 23.773 & 26.098 & 26.132 & 29.656 & 29.812 & 23.014 & 23.047 \\
\hline \multirow[b]{2}{*}{2} & Head & 6.79 & 7.2 & 6.653 & 6.683 & 7.312 & 7.435 & 5.971 & 6.005 \\
\hline & Tails & 23.97 & 24.052 & 25.726 & 25.737 & 28.046 & 28.101 & 23.682 & 23.704 \\
\hline \multirow[b]{2}{*}{3} & Head & 8.97 & 9.01 & 6.482 & 6.502 & 6.183 & 6.217 & 5.642 & 5.702 \\
\hline & Tails & 23.491 & 23.512 & 30.544 & 30.55 & 27.11 & 27.138 & 22.117 & 22.14 \\
\hline \multirow[b]{2}{*}{4} & Head & 9.178 & 9.254 & 6.437 & 6.46 & 6.726 & 6.751 & 5.633 & 5.635 \\
\hline & Tails & 23.622 & 23.734 & 25.125 & 25.146 & 27.252 & 27.342 & 21.55 & 21.559 \\
\hline \multirow[b]{2}{*}{5} & Head & 6.941 & 7.009 & 6.462 & 6.467 & 6.662 & 6.678 & 5.838 & 5.855 \\
\hline & Tails & 23.121 & 23.162 & 25.174 & 25.178 & 27.242 & 27.248 & 21.768 & 21.778 \\
\hline \multirow[b]{2}{*}{6} & Head & 6.583 & 6.612 & 7.111 & 7.136 & 6.917 & 7.001 & 5.737 & 5.766 \\
\hline & Tails & 23.634 & 23.655 & 28.612 & 28.617 & 27.987 & 28.023 & 22.019 & 22.04 \\
\hline \multirow[b]{2}{*}{7} & Head & 6.679 & 6.706 & 7.438 & 7.445 & 6.446 & 6.523 & 6.128 & 6.2 \\
\hline & Tails & 23.664 & 23.683 & 26.35 & 26.45 & 27.828 & 27.903 & 22.69 & 22.708 \\
\hline \multirow{6}{*}{ Sensor no. } & & Batch S & & Batch 6 & & Batch 7 & & Batch 8 & \\
\hline & & Speed (rpm) & 1500 & Speed (rpm) & 1500 & Speed (rpm) & 1500 & Speed (rpm) & 1500 \\
\hline & & Ratio (PEDOT/Methanol) & 1 & Ratio (PEDOT/Methanoi) & 2 & Ratio (PEDOT/Methanol) & 1 & Ratio (PEDOT/Methano & 2 \\
\hline & & Concentration $\%$ & 0.8 & Concentration \% & 0.8 & Concentration \% & 4 & Concentration \% & 4 \\
\hline & & Min Res. & Max Res. & Min Res. & Max Res. & Min Res. & Max Res. & Min Res. & Max Res. \\
\hline & Head & 8.03 & 8.197 & 55.808 & 55.843 & 7.201 & 7.398 & 6.14 & 6.175 \\
\hline 1 & Tails & 23.661 & 23.674 & 71.561 & 71.577 & 25.759 & 25.768 & 22.97 & 22.987 \\
\hline \multirow[b]{2}{*}{2} & Head & 6.771 & 6.798 & 6.854 & 6.904 & 6.754 & 6.767 & 6.743 & 6.851 \\
\hline & Tails & 24.094 & 24.102 & 22.491 & 22.499 & 25.407 & 25.447 & 22.729 & 22.802 \\
\hline \multirow[b]{2}{*}{3} & Head & 6.512 & 6.543 & 6.254 & 6.264 & 6.341 & 6.355 & 6.542 & 6.836 \\
\hline & Tails & 22.863 & 22.886 & 21.713 & 21.715 & 24.743 & 24.753 & 21.574 & 21.603 \\
\hline \multirow[b]{2}{*}{4} & Head & 6.299 & 6.318 & 6.34 & 6.393 & 6.257 & 6.263 & 6.011 & 6.074 \\
\hline & Tails & 22.519 & 22.525 & 21.491 & 21.493 & 24.693 & 26.707 & 22.312 & 22.507 \\
\hline \multirow[b]{2}{*}{5} & Head & 6.403 & 6.407 & 6.249 & 6.256 & 6.371 & 6.417 & 6.061 & 6.093 \\
\hline & Tails & 22.834 & 22.838 & 22.133 & 22.156 & 25.624 & 25.512 & 22.477 & 22.494 \\
\hline \multirow{4}{*}{6} & Head & 6.691 & 6.742 & 6.6 & 6.622 & 6.348 & 6.361 & 6.38 & 6.396 \\
\hline & Tails & 23.39 & 23.402 & 22.615 & 22.623 & 25.445 & 25.468 & 23.731 & 23.807 \\
\hline & Head & 7.712 & 7.742 & 6.462 & 6.478 & 6.517 & 6.567 & 6.212 & 6.324 \\
\hline & Tails & 24.453 & 24.48 & 22.937 & 22.947 & 27.404 & 27.689 & 22.787 & 22.803 \\
\hline
\end{tabular}

Figure 5.4. Resistances measured on skin sensor heads and tails with 8 batches of recipes of PEDOT:PSS coating 

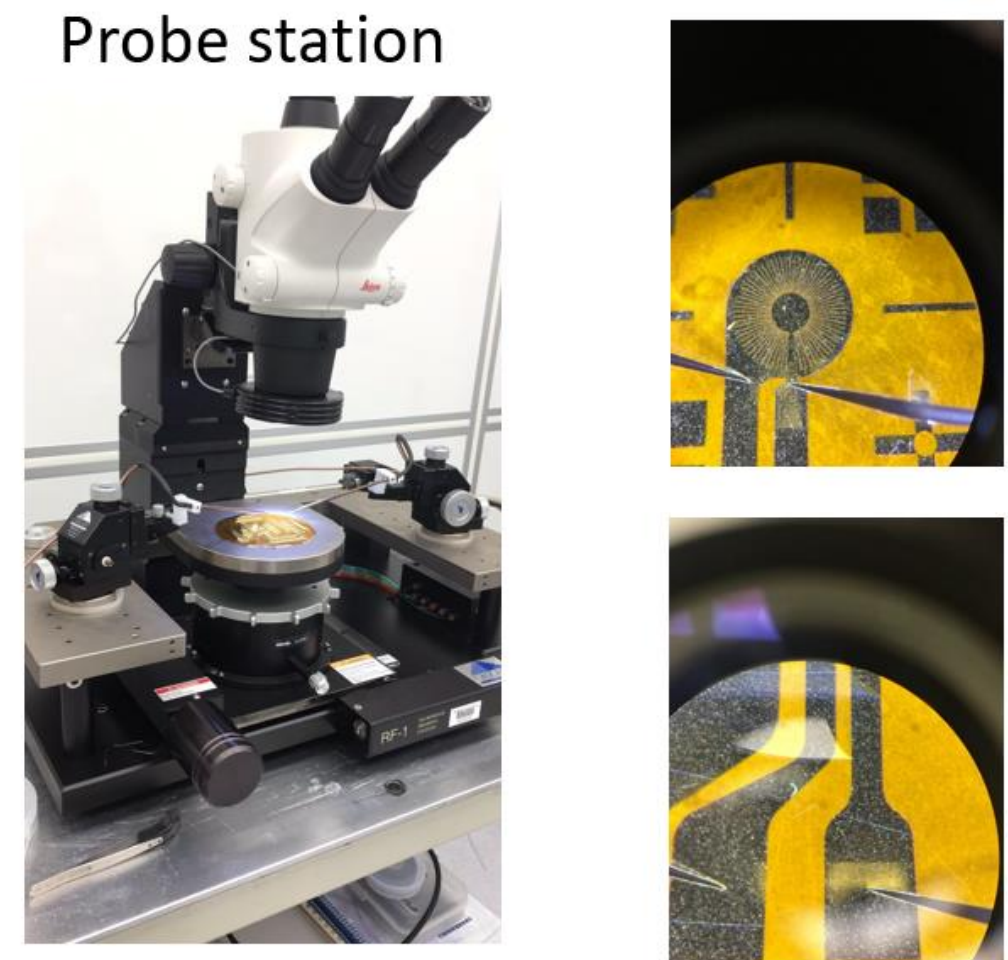

Head

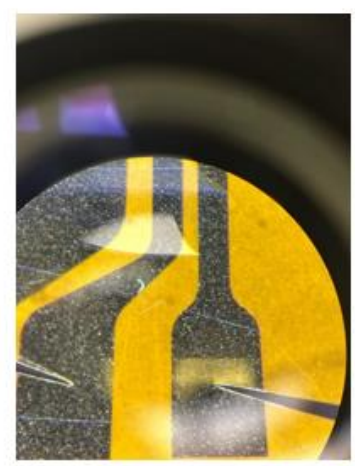

Tails

Figure 5.5. Probe station for skin sensor resistance measurement on head and tails 


\section{CHAPTER 6}

\section{CONCLUSION AND FUTURE WORK}

\subsection{Conclusion}

Generally, a large part of this research focused on the control system for driving and assembling microrobots. In addition, for extension of MEMS applications to robotics, I fabricated distributed arrays of flexible skin sensors, and measured their sheet resistance after coating the sensors with a PEDOT:PSS thin film.

The first microrobot that I investigated in this research was a magnetic field actuated microrobot used for the MMC2017 competition. It is a 250 microns diameter chrome-steel sphere driven by a single conical permanent magnet. Using this technique, the microrobot can be untethered moving following in manipulation.

The second microrobot studied was an assembled MEMS microrobots named the AFAM. The microrobot was simulated by FEA software, and successfully assembled using a custom NeXus microassembly system. The microassembly system has two manipulators, M1 and M2, and is operated via calibration, inverse kinematics, and visual closed-loop control methods. M1 is the manipulator carrying the MEMS substrate, while M2 is the manipulator carrying the microgripper. These schemes ensure coarse and fine adjustments for M1, as well as rotation centering and a 3-point teaching method for M2. These innovative techniques are necessary for the microassembly system to realize semiautomation capabilities. 
The final contribution of the thesis was in developing of cleanroom recipes for fabrication and lamination of distributed MEMS sensors. A novel wet lift-off photolithographic technique was developed for PEDOT: PSS coatings and used for prototype sensors and arrays. Preliminary evaluations have been made to prove the feasibility of the fabrication method.

\subsection{Future work}

In the future, we will explore more applications of microrobots, and new design structures that are capable of assembly. Microrobot simulations will be used to guide improvements in SAFAM components and to fabricate SOI microrobots in the cleanroom. Meanwhile, the NeXus microassembly system will be updated and improved toward full automation. An extra manipulator will be added to in the microassembly system for further applications such as probing and epoxy dispensing. Finally, for the flexible robotic skin sensor, there are still challenges to address, such as increasing the sensor sensitivity to strain, and decreasing of electrical crosstalk via different PEDOT:PSS solutions formulations. 


\section{REFERENCES}

[1] J. Edd, S. Payen, B. Rubinsky, M. L. Stoller, and M. Sitti, "Biomimetic propulsion for a swimming surgical micro-robot," in Intelligent Robots and Systems, 2003.(IROS 2003). Proceedings. 2003 IEEE/RSJ International Conference on, 2003, vol. 3, pp. 2583-2588: IEEE.

[2] D. Oleynikov, S. Farritor, A. Hadzialic, and S. R. Platt, "Microrobot for surgical applications," ed: Google Patents, 2006.

[3] M. P. Kummer, J. J. Abbott, B. E. Kratochvil, R. Borer, A. Sengul, and B. J. Nelson, "OctoMag: An electromagnetic system for 5-DOF wireless micromanipulation," IEEE Transactions on Robotics, vol. 26, no. 6, pp. 1006-1017, 2010.

[4] R. Murthy and D. O. Popa, "Millimeter-scale microrobots for wafer-level factories," in Robotics and Automation (ICRA), 2010 IEEE International Conference on, 2010, pp. 488493: IEEE.

[5] T. Inoue, K. Iwatani, I. Shimoyama, and H. Miura, "Micromanipulation using magnetic field," in Robotics and Automation, 1995. Proceedings., 1995 IEEE International Conference on, 1995, vol. 1, pp. 679-684: IEEE.

[6] S. N. Tabatabaei, S. Duchemin, H. Girouard, and S. Martel, "Towards MR-navigable nanorobotic carriers for drug delivery into the brain," in Robotics and Automation (ICRA), 2012 IEEE International Conference on, 2012, pp. 727-732: IEEE.

[7] S. Wagner et al., "Electronic skin: architecture and components," Physica E: Lowdimensional Systems and Nanostructures, vol. 25, no. 2-3, pp. 326-334, 2004.

[8] C. Wang et al., "User-interactive electronic skin for instantaneous pressure visualization," Nature materials, vol. 12, no. 10, p. 899, 2013.

[9] D. Vogt et al., "Progress in soft, flexible, and stretchable sensing systems," in Proceedings of the International Workshop on Research Frontiers in Electronics Skin Technology at ICRA, 2013, vol. 13.

[10] A. Billard et al., "The roboskin project: Challenges and results," in Romansy 19-Robot Design, Dynamics and Control: Springer, 2013, pp. 351-358.

[11] C. H. Lin, T. W. Erickson, J. A. Fishel, N. Wettels, and G. E. Loeb, "Signal processing and fabrication of a biomimetic tactile sensor array with thermal, force and microvibration modalities," in Robotics and Biomimetics (ROBIO), 2009 IEEE International Conference on, 2009, pp. 129-134: IEEE.

[12] M. L. Hammock, A. Chortos, B. C. K. Tee, J. B. H. Tok, and Z. Bao, "25th anniversary article: the evolution of electronic skin (e-skin): a brief history, design considerations, and recent progress," Advanced materials, vol. 25, no. 42, pp. 5997-6038, 2013.

[13] J. R. Baptist, R. Zhang, D. Wei, M. N. Saadatzi, and D. O. Popa, "Fabrication of strain gauge based sensors for tactile skins," in Smart Biomedical and Physiological Sensor Technology XIV, 2017, vol. 10216, p. 102160F: International Society for Optics and Photonics. 
[14] G. Hwang et al., "Mobile microrobotic manipulator in microfluidics," Sensors and Actuators A: Physical, vol. 215, pp. 56-64, 2014.

[15] E. Diller, S. Floyd, C. Pawashe, and M. Sitti, "Control of multiple heterogeneous magnetic microrobots in two dimensions on nonspecialized surfaces," IEEE Transactions on Robotics, vol. 28, no. 1, pp. 172-182, 2012.

[16] E. Diller, S. Floyd, C. Pawashe, and M. Sitti, "Control of multiple heterogeneous magnetic micro-robots on non-specialized surfaces," in Robotics and Automation (ICRA), 2011 IEEE International Conference on, 2011, pp. 115-120: IEEE.

[17] C. Pawashe, S. Floyd, and M. Sitti, "Multiple magnetic microrobot control using electrostatic anchoring," Applied Physics Letters, vol. 94, no. 16, p. 164108, 2009.

[18] S. Floyd, C. Pawashe, and M. Sitti, "An untethered magnetically actuated micro-robot capable of motion on arbitrary surfaces," in Robotics and Automation, 2008. ICRA 2008. IEEE International Conference on, 2008, pp. 419-424: IEEE.

[19] J. A. Walraven, "Introduction to Applications and Industries for Microelectromechanical Systems (MEMS)," in ITC, 2003, pp. 674-680.

[20] F. Khoshnoud and C. W. de Silva, "Recent advances in MEMS sensor technologymechanical applications," IEEE Instrumentation \& Measurement Magazine, vol. 15, no. 2, 2012.

[21] R. J. Pryputniewicz, R. T. Marinis, A. R. Klempner, and P. Hefti, "Hybrid methodology for development of MEMS," in Position, Location, And Navigation Symposium, 2006 IEEE/ION, 2006, pp. 606-613: IEEE.

[22] C. Nothnagle, J. R. Baptist, J. Sanford, W. H. Lee, D. O. Popa, and M. B. Wijesundara, "EHD printing of PEDOT: PSS inks for fabricating pressure and strain sensor arrays on flexible substrates," in Next-Generation Robotics II; and Machine Intelligence and Bioinspired Computation: Theory and Applications IX, 2015, vol. 9494, p. 949403 : International Society for Optics and Photonics.

[23] J. Shin, W. H. Lee, C. Nothnagle, and M. B. Wijesundara, "EHD as sensor fabrication technology for robotic skins," in Next-Generation Robots and Systems, 2014, vol. 9116, p. 91160F: International Society for Optics and Photonics.

[24] F. Mirza, R. R. Sahasrabuddhe, J. R. Baptist, M. B. Wijesundara, W. H. Lee, and D. O. Popa, "Piezoresistive pressure sensor array for robotic skin," in Sensors for NextGeneration Robotics III, 2016, vol. 9859, p. 98590K: International Society for Optics and Photonics.

[25] S. K. Das, J. R. Baptist, R. Sahasrabuddhe, W. H. Lee, and D. O. Popa, "Package analysis of 3D-printed piezoresistive strain gauge sensors," in Sensors for Next-Generation Robotics III, 2016, vol. 9859, p. 985905: International Society for Optics and Photonics.

[26] E. J. Wilson, "Strain-gage instrumentation," Harris'Shock and Vibration Handbook, S, pp. 17.1-17.15, 1976.

[27] G. Schwartz et al., "Flexible polymer transistors with high pressure sensitivity for application in electronic skin and health monitoring," Nature communications, vol. 4, p. 1859, 2013.

[28] G. Latessa, F. Brunetti, A. Reale, G. Saggio, and A. Di Carlo, "Piezoresistive behaviour of flexible PEDOT: PSS based sensors," Sensors and Actuators B: Chemical, vol. 139, no. 2, pp. 304-309, 2009.

[29] U. Lang, P. Rust, B. Schoberle, and J. Dual, "Piezoresistive properties of PEDOT: PSS," Microelectronic Engineering, vol. 86, no. 3, pp. 330-334, 2009.

[30] M. Muraki, S. Takamatsu, K. Matsumoto, and I. Shimoyama, "Organic semiconductor based strain sensors for input system on flexible oleds," in Micro Electro Mechanical 
Systems, 2008. MEMS 2008. IEEE 21st International Conference on, 2008, pp. 904-907: IEEE.

[31] U. Lang, P. Rust, and J. Dual, "Towards fully polymeric MEMS: Fabrication and testing of PEDOT/PSS strain gauges," Microelectronic Engineering, vol. 85, no. 5-6, pp. 1050-1053, 2008.

[32] R. Mateiu, M. Lillemose, T. S. Hansen, A. Boisen, and O. Geschke, "Reliability of poly 3, 4ethylenedioxythiophene strain gauge," Microelectronic Engineering, vol. 84, no. 5-8, pp. 1270-1273, 2007.

[33] V. Correia, C. Caparros, C. Casellas, L. Francesch, J. Rocha, and S. Lanceros-Mendez, "Development of inkjet printed strain sensors," Smart Materials and Structures, vol. 22, no. 10, p. 105028, 2013.

[34] J. A. DeFranco, B. S. Schmidt, M. Lipson, and G. G. Malliaras, "Photolithographic patterning of organic electronic materials," Organic Electronics, vol. 7, no. 1, pp. 22-28, 2006.

[35] S. Ouyang et al., "Surface patterning of PEDOT: PSS by photolithography for organic electronic devices," Journal of Nanomaterials, vol. 2015, p. 4, 2015.

[36] S. Takamatsu and T. Itoh, "Novel MEMS Devices Based on Conductive Polymers," The Electrochemical Society Interface, vol. 21, no. 3-4, pp. 63-66, 2012.

[37] S. Takamatsu, T. Takahata, M. Muraki, E. Iwase, K. Matsumoto, and I. Shimoyama, "Transparent conductive-polymer strain sensors for touch input sheets of flexible displays," Journal of Micromechanics and Microengineering, vol. 20, no. 7, p. 075017, 2010.

[38] A. Elschner, W. Loevenich, A. Eiling, and J. Bayley, "ITO Alternative: solution deposited Clevios TM PEDOT: PSS for transparent conductive applications," Heraeus trade article, 2012.

[39] M. Kuş and S. Okur, "Electrical characterization of PEDOT: PSS beyond humidity saturation," Sensors and Actuators B: Chemical, vol. 143, no. 1, pp. 177-181, 2009.

[40] O. Dimitriev, D. Grinko, Y. V. Noskov, N. Ogurtsov, and A. Pud, "PEDOT: PSS films-Effect of organic solvent additives and annealing on the film conductivity," Synthetic metals, vol. 159, no. 21-22, pp. 2237-2239, 2009.

[41] S. Guo, T. Fukuda, and K. Asaka, "A new type of fish-like underwater microrobot," leee/Asme Transactions on Mechatronics, vol. 8, no. 1, pp. 136-141, 2003.

[42] Q. Pan and S. Guo, "Development of the novel types of biomimetic microrobots driven by external magnetic field," in Robotics and Biomimetics, 2007. ROBIO 2007. IEEE International Conference on, 2007, pp. 256-261: IEEE.

[43] W. Zhang, S. Guo, and K. Asaka, "Development of an underwater biomimetic microrobot with compact structure and flexible locomotion," Microsystem technologies, vol. 13, no. 8-10, pp. 883-890, 2007.

[44] J. J. Abbott et al., "How should microrobots swim?," The international journal of Robotics Research, vol. 28, no. 11-12, pp. 1434-1447, 2009.

[45] L. Zhang, J. J. Abbott, L. Dong, B. E. Kratochvil, D. Bell, and B. J. Nelson, "Artificial bacterial flagella: Fabrication and magnetic control," Applied Physics Letters, vol. 94, no. 6, p. 064107, 2009.

[46] S. Floyd, C. Pawashe, and M. Sitti, "Microparticle manipulation using multiple untethered magnetic micro-robots on an electrostatic surface," in Intelligent Robots and Systems, 2009. IROS 2009. IEEE/RSJ International Conference on, 2009, pp. 528-533: IEEE. 
[47] C. Pawashe, S. Floyd, and M. Sitti, "Modeling and experimental characterization of an untethered magnetic micro-robot," The International Journal of Robotics Research, vol. 28, no. 8, pp. 1077-1094, 2009.

[48] K. B. Yesin, K. Vollmers, and B. J. Nelson, "Modeling and control of untethered biomicrorobots in a fluidic environment using electromagnetic fields," The International Journal of Robotics Research, vol. 25, no. 5-6, pp. 527-536, 2006.

[49] S. Sudo, S. Segawa, and T. Honda, "Magnetic swimming mechanism in a viscous liquid," Journal of intelligent material systems and structures, vol. 17, no. 8-9, pp. 729-736, 2006.

[50] R. J. Wood, "Design, fabrication, and analysis of a 3DOF, 3cm flapping-wing MAV," in Intelligent Robots and Systems, 2007. IROS 2007. IEEE/RSJ International Conference on, 2007, pp. 1576-1581: IEEE.

[51] R. J. Wood, S. Avadhanula, M. Menon, and R. S. Fearing, "Microrobotics using composite materials: The micromechanical flying insect thorax," in Robotics and Automation, 2003. Proceedings. ICRA'03. IEEE International Conference on, 2003, vol. 2, pp. 1842-1849: IEEE.

[52] M. Lok, X. Zhang, E. F. Helbling, R. Wood, D. Brooks, and G.-Y. Wei, "A power electronics unit to drive piezoelectric actuators for flying microrobots," in Custom Integrated Circuits Conference (CICC), 2015 IEEE, 2015, pp. 1-4: IEEE.

[53] K. Y. Ma, P. Chirarattananon, and R. J. Wood, "Design and fabrication of an insect-scale flying robot for control autonomy," in Intelligent Robots and Systems (IROS), 2015 IEEE/RSJ International Conference on, 2015, pp. 1558-1564: IEEE.

[54] Y. Chen, E. F. Helbling, N. Gravish, K. Ma, and R. J. Wood, "Hybrid aerial and aquatic locomotion in an at-scale robotic insect," in Intelligent Robots and Systems (IROS), 2015 IEEE/RSJ International Conference on, 2015, pp. 331-338: IEEE.

[55] R. Murthy, A. N. Das, D. O. Popa, and H. E. Stephanou, "ARRIpede: An assembled diescale microcrawler," Advanced Robotics, vol. 25, no. 8, pp. 965-990, 2011.

[56] X. Huang, X. Lv, and M. Wang, "Development of a robotic microassembly system with multi-manipulator cooperation," in Mechatronics and Automation, Proceedings of the 2006 IEEE International Conference on, 2006, pp. 1197-1201: IEEE.

[57] R. L. Hollis and A. A. Rizzi, "Agile assembly architecture: A platform technology for microassembly," in Proc. Am. Soc. for Precision Engineering 19th Annual Meeting, Orlando, 2004.

[58] M. B. Cohn et al., "Microassembly technologies for MEMS," in Microelectronic Structures and MEMS for Optical Processing IV, 1998, vol. 3513, pp. 2-17: International Society for Optics and Photonics.

[59] M. Probst, C. Hürzeler, R. Borer, and B. J. Nelson, "A microassembly system for the flexible assembly of hybrid robotic MEMS devices," International Journal of Optomechatronics, vol. 3, no. 2, pp. 69-90, 2009.

[60] Zyvex. http://www.zyvex.com/Products/home.html.

[61] ICRA, "Mobile Microrobot Challenge 2017," 2017.

[62] N. A. Torres and D. O. Popa, "Cooperative control of multiple untethered magnetic microrobots using a single magnetic field source," in Automation Science and Engineering (CASE), 2015 IEEE International Conference on, 2015, pp. 1608-1613: IEEE.

[63] N. A. Torres, S. Ruggeri, and D. O. Popa, "Untethered microrobots actuated with focused permanent magnet field," in ASME 2014 International Design Engineering Technical Conferences and Computers and Information in Engineering Conference, 2014, pp. V004T09A024-V004T09A024: American Society of Mechanical Engineers. 
[64] R. a. A. Society. (2017). IEEE RAS Micro/Nano Robotics \& Automation: Mobile Microrobotis Challenge.

[65] R. Murthy, H. E. Stephanou, and D. O. Popa, "AFAM: An articulated four axes microrobot for nanoscale applications," IEEE Transactions on Automation Science and Engineering, vol. 10, no. 2, pp. 276-284, 2013.

[66] A. N. Das, P. Zhang, W. H. Lee, D. Popa, and H. Stephanou, " $\mu$ 3: multiscale, deterministic micro-nano assembly system for construction of on-wafer microrobots," in Robotics and Automation, 2007 IEEE International Conference on, 2007, pp. 461-466: IEEE.

[67] H. Koichi and H. Tom, Visual servoing: real-time control of robot manipulators based on visual sensory feedback. World scientific, 1993.

[68] S. Hutchinson, G. D. Hager, and P. I. Corke, "A tutorial on visual servo control," IEEE transactions on robotics and automation, vol. 12, no. 5, pp. 651-670, 1996.

[69] Y. Fang, D. Dawson, W. Dixon, and M. Dequeiroz, "2.5 D visual servoing of wheeled mobile robots," in Proc. of the IEEE Conference on Decision and Control, Las Vegas, NV, 2002: Citeseer. 


\section{APPENDIX A - LabVIEW Schematics}

NeXus microassembly system LabVIEW schematics

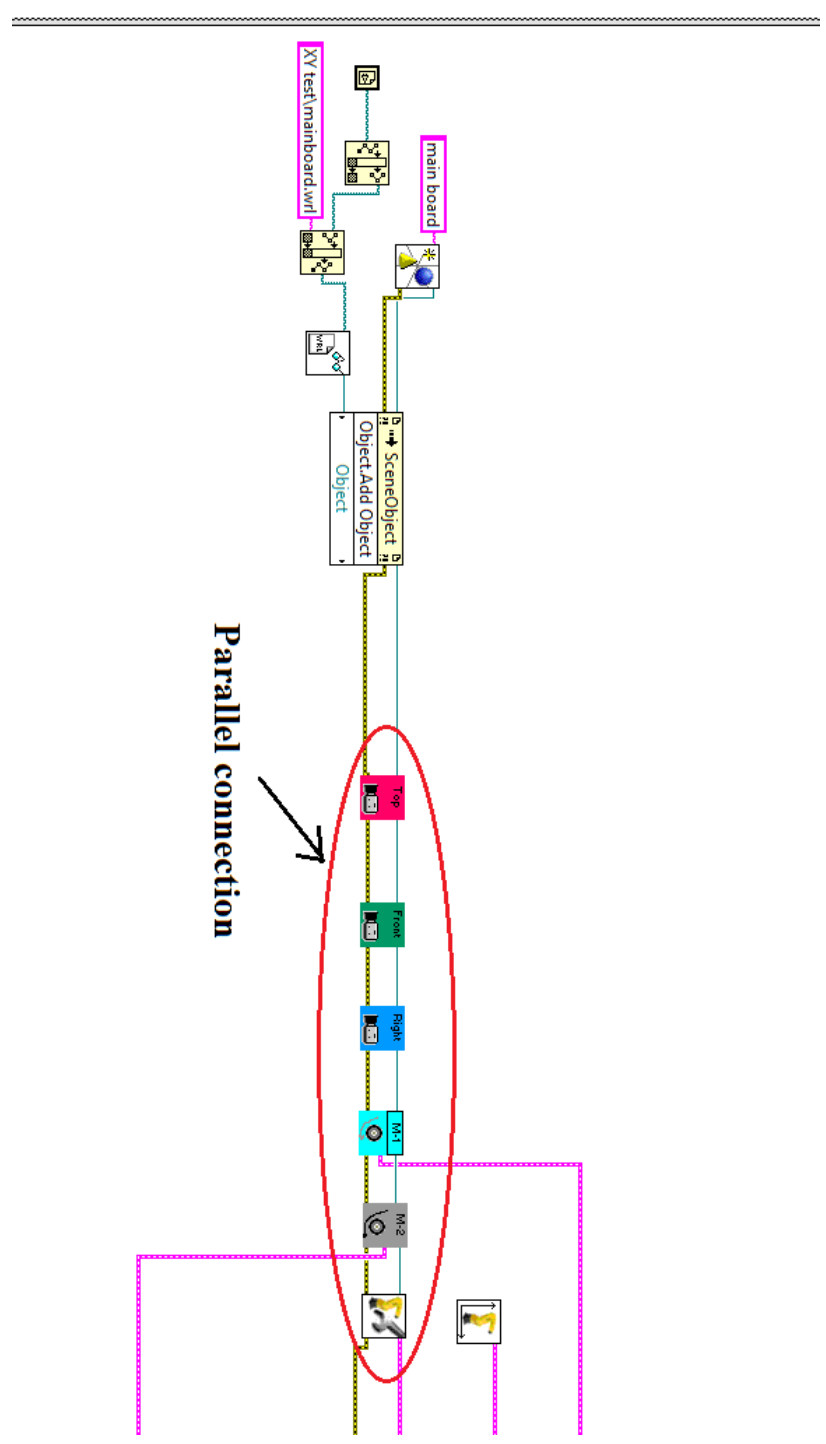

Figure A.1. SubVIs of top, front, and side camera connected in parallel with M1, M2 and DENSO 


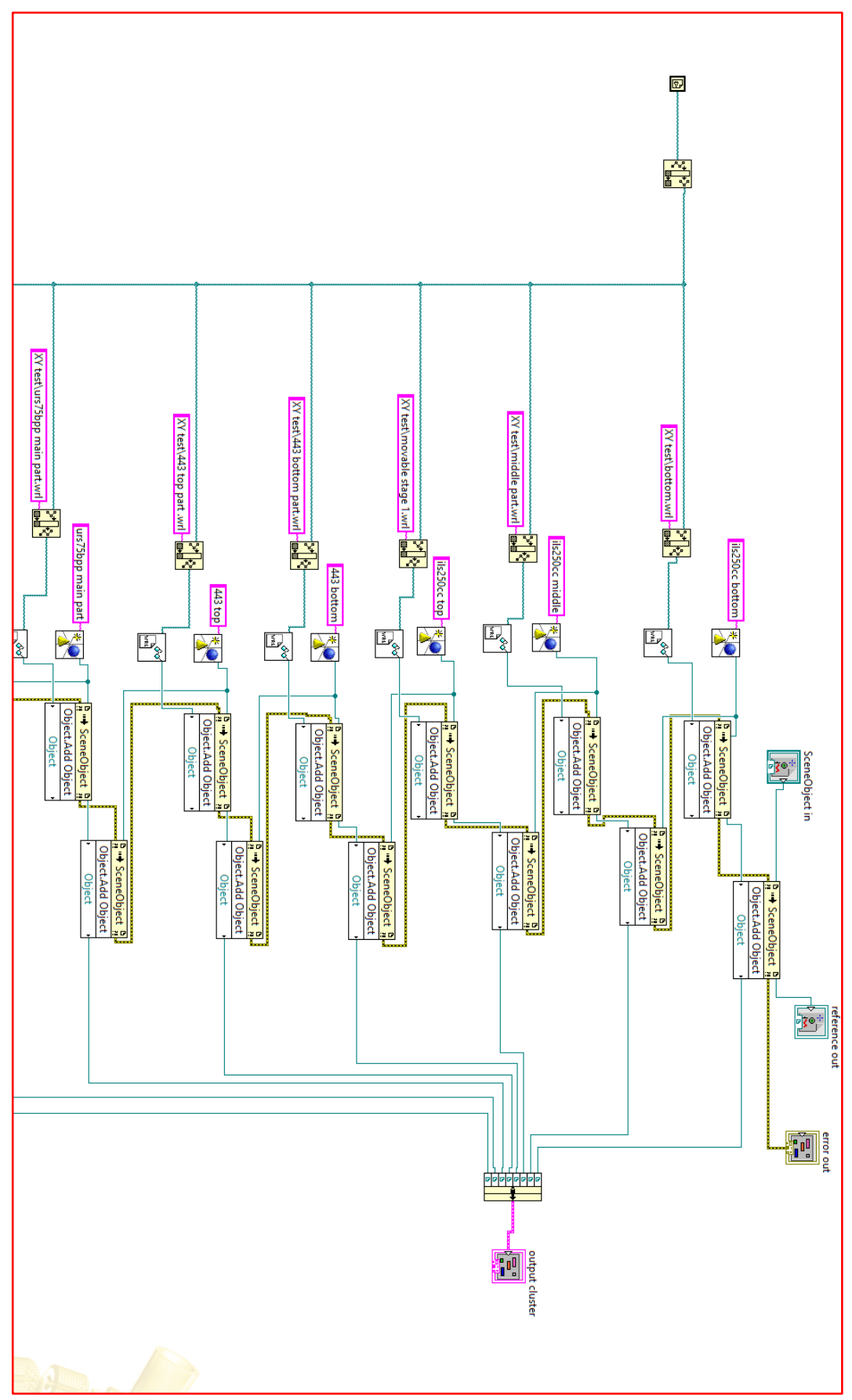

Figure A.2. SubVI of manipulator 1 


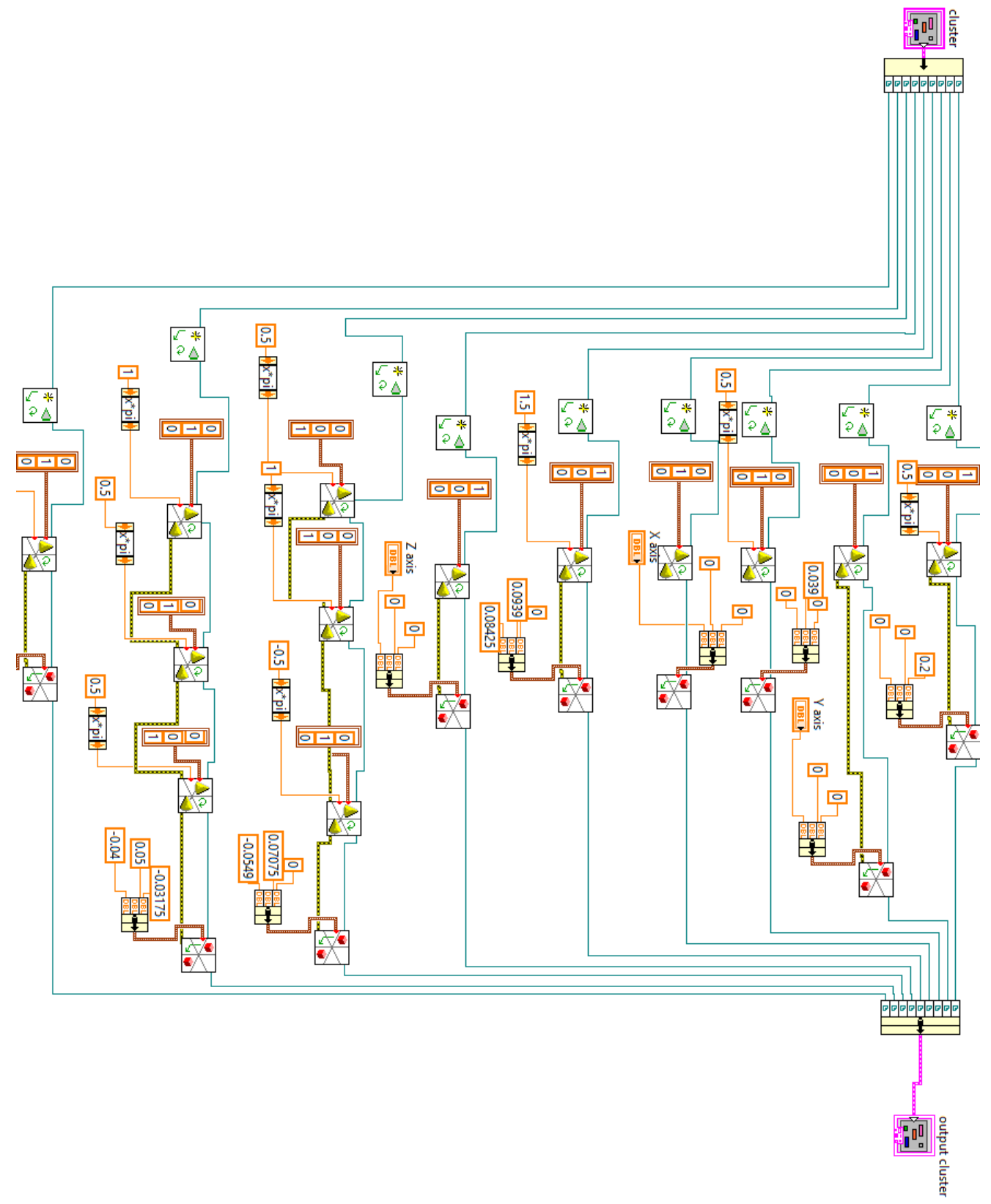

Figure A.3. SubVI of manipulator 1 manual control 


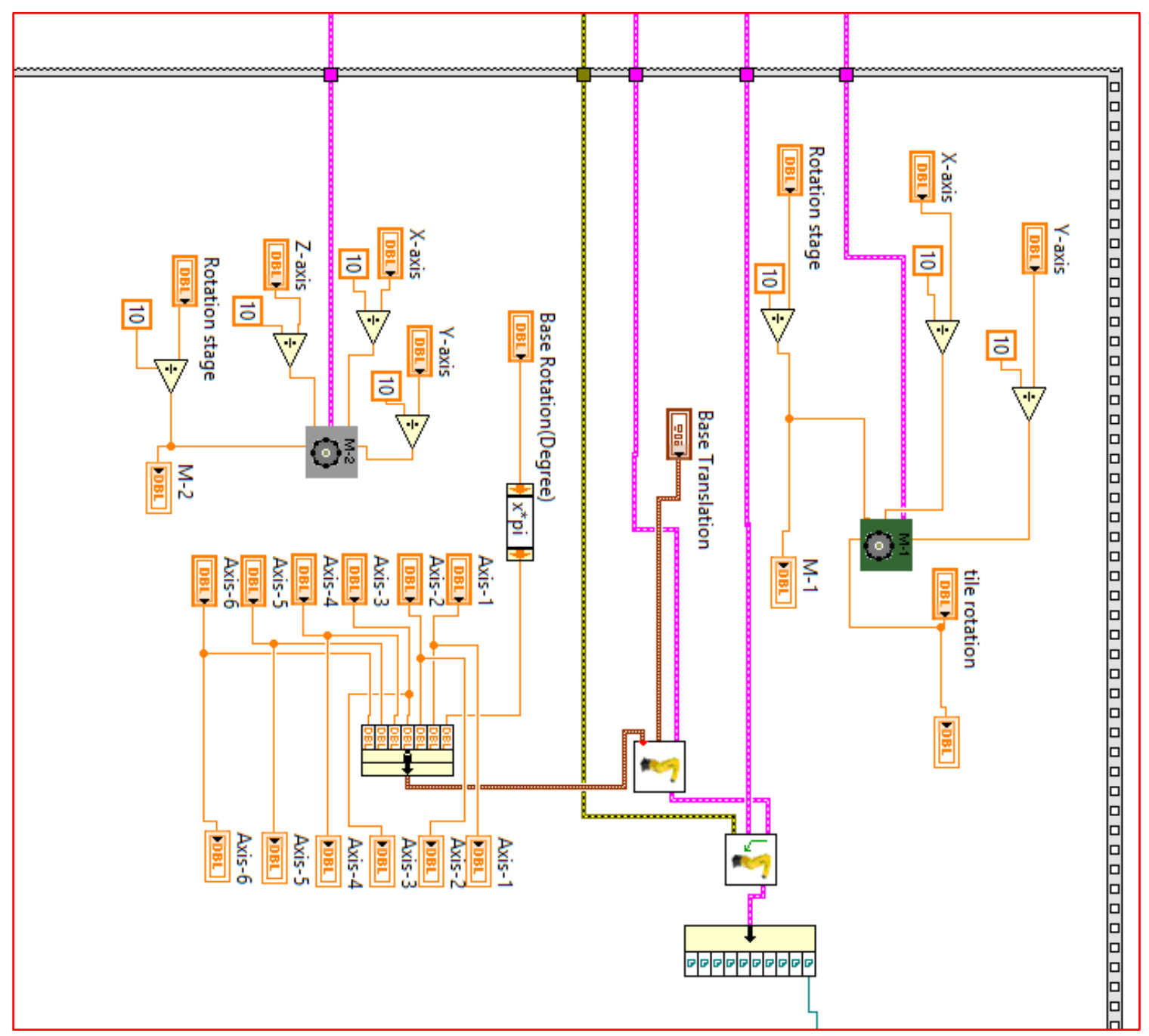

Figure A.4. M1, M2, and DENSO manual controls 


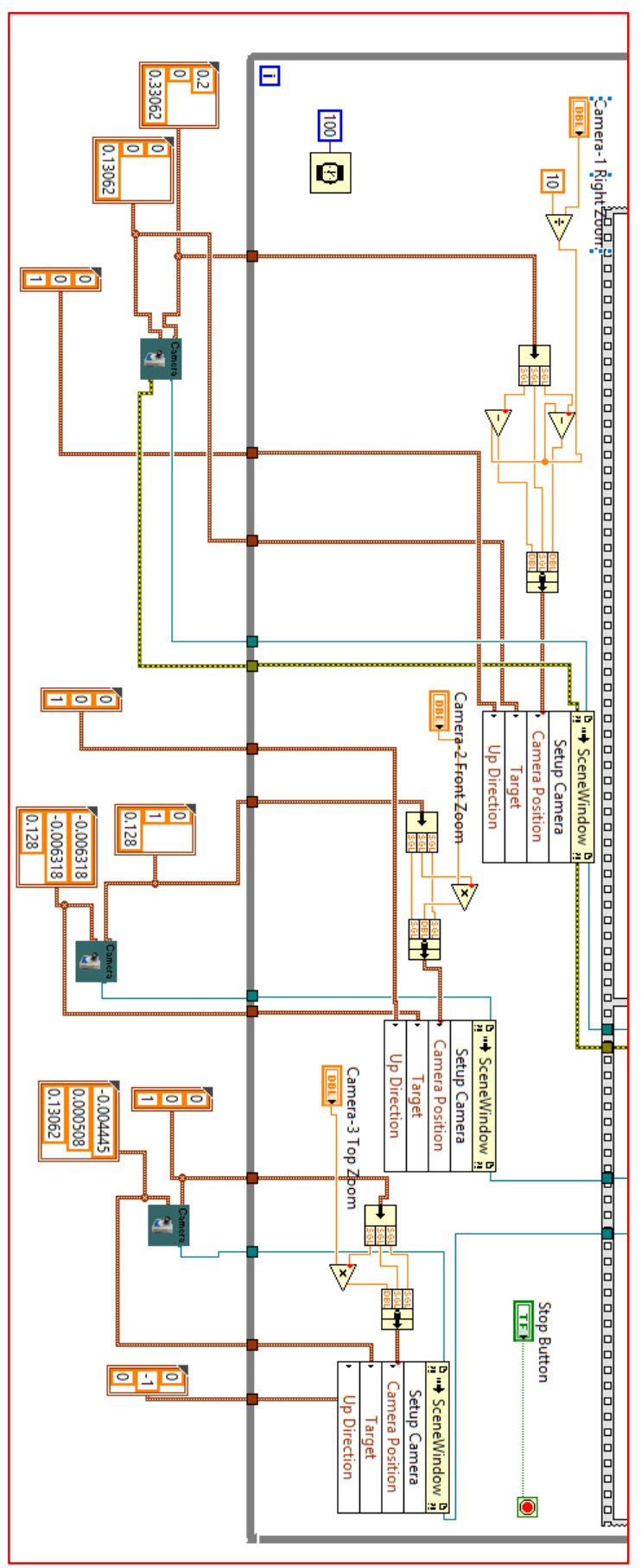

Figure A.5. Three imaging system cameras setup 


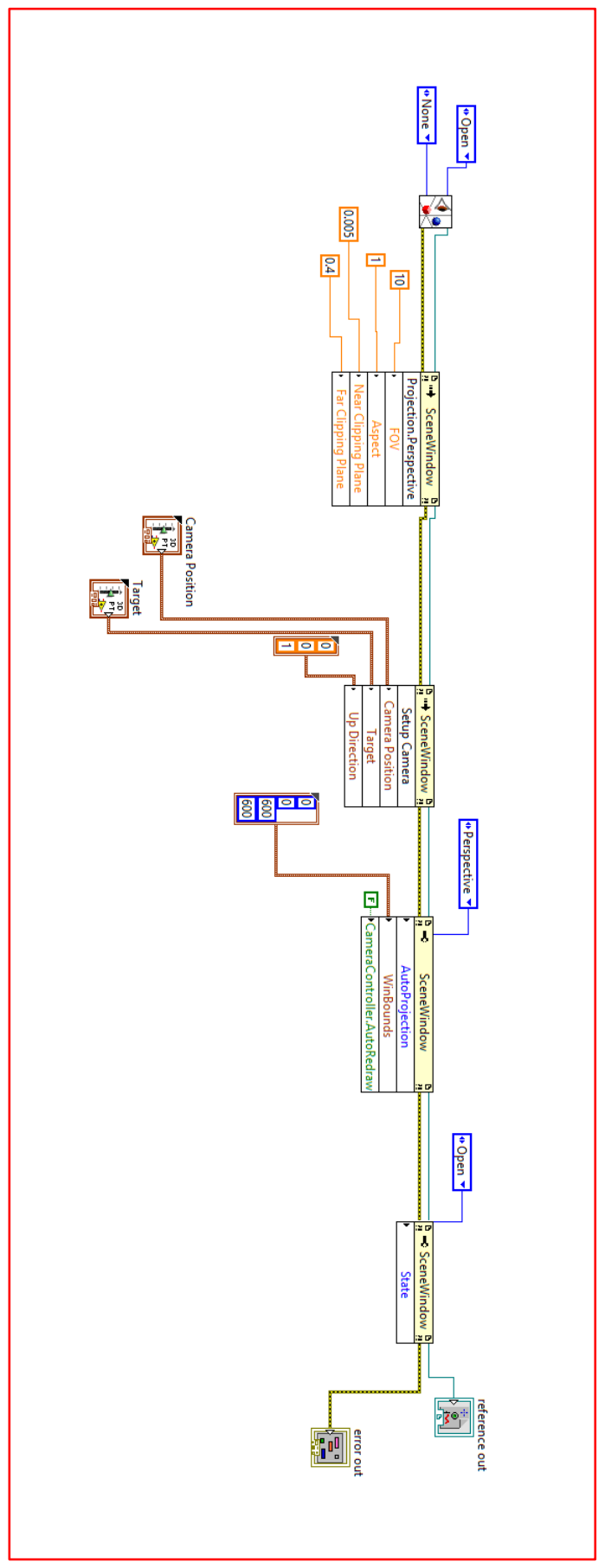

Figure A.6. SubVI of camera parameter 


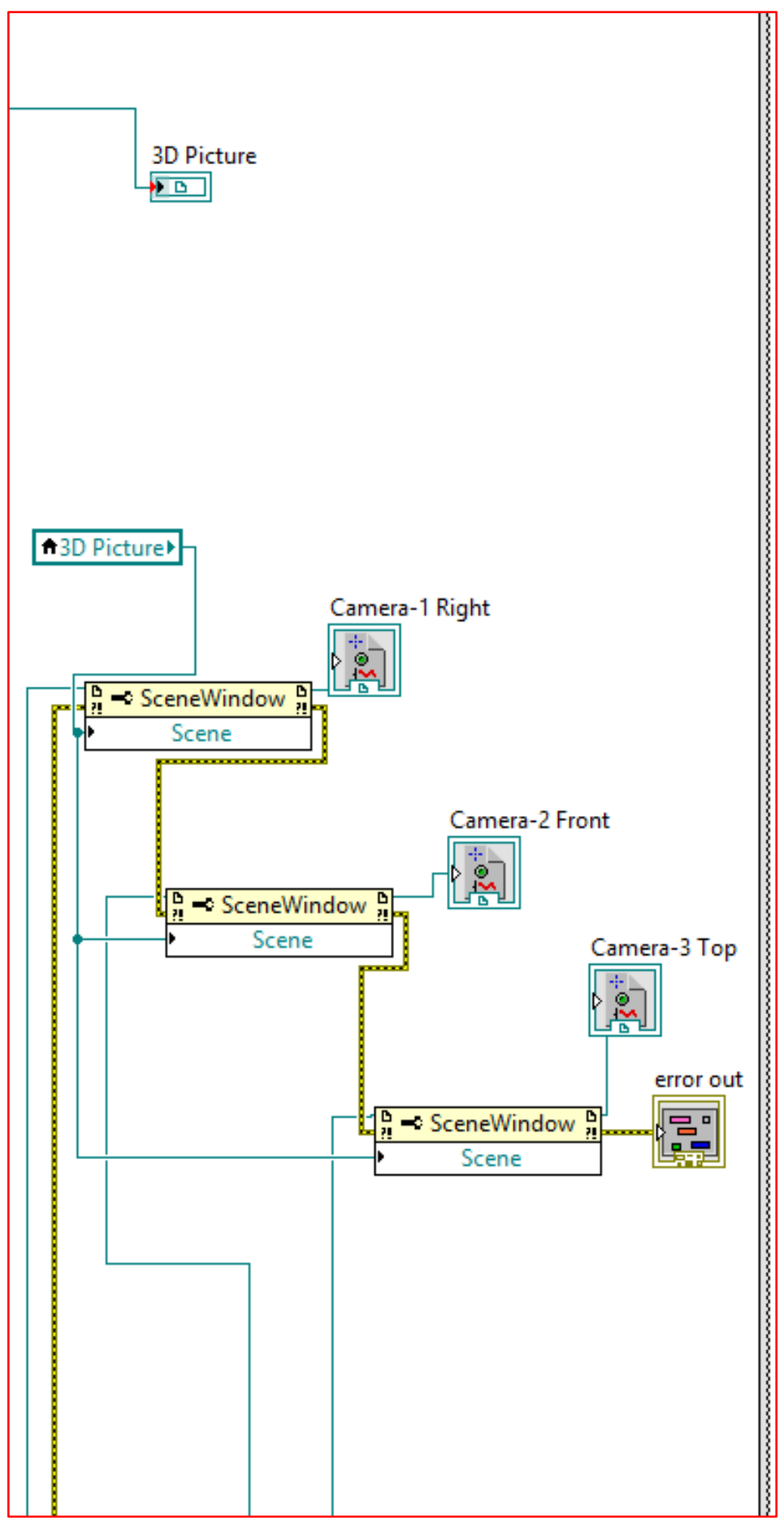

Figure A.7. Images generation 


\section{APPENDIX B - Flexible skin sensor receipt [13]}

Step 1: First, a clean Silicon carrier wafer is obtained and coated with MicroChem SPR220-3.0 photoresist.

Step 2: A stock sheet of Kapton obtained from McMaster Carr is cut to an appropriate size and cleaned with Acetone and Isopropyl alcohol. This is then aligned on the carrier and transferred to a hotplate at 115 degrees Celsius, where the Kapton is covered with a cleanroom wipe and laminated using a brayer. This wafer is removed and allowed to cool. The Kapton is then blown with N2 to remove any particulates. A bi-layer photoresist composed of MicroChem LOR10B and SPR220-3.0 were spun onto the wafer for patterning the electrodes.

Step 3: After exposure, the wafer is hard baked at 115 Celsius for 60 seconds, then the wafer is loaded into a mask aligner and exposed for 11 seconds.

Step 4: The wafer is then post-exposure-baked at 115 Celsius for 60 seconds and developed, dried, and cleaned using Reactive Ion Etching (RIE) set at 50 watts with a 20 SCCM flow rate of Oxygen for 45 seconds.

Step 5: The carrier wafer and patterned Kapton is then transferred to a sputter deposition system (Lesker PVD 75) where $300 \mathrm{~nm}$ of Gold is deposited. The coated wafer and substrate are then placed in an Acetone liftoff bath where it is sits in a sonicated bath for approximately 20 minutes. The substrate detaches from the carrier and is rinsed several 
times with Acetone and Isopropyl alcohol before further processing. The Kapton sheet containing the interdigitated structures then adheres to a new carrier wafer following the same procedure as previously mentioned. Following this step, substrates are again cleaned with $\mathrm{N} 2$ and RIE.

Step 6: Next, a single layer of thin 1805 photoresist is spun onto the devices, hard-baked and exposed in a mask aligner. The wafer is then post-exposure-baked, developed, dried, and cleaned using Reactive Ion Etching (RIE) in a lower power oxygen plasma.

Step 7: Next the PEDOT: PSS based solution is spun onto the wafer at $2000 \mathrm{rpm}$, now with windows over the interdigitated structures. The wafer is then allowed to dry under vacuum in a convection oven. Finally, the wafer is transferred to a lift-off bath containing Acetone and allowed to sit for about 15 minutes while undergoing agitation. After the Kapton departs the carrier it is rinsed several times with acetone and IPA. The PEDOT: PSS is now patterned over the ID structures and microfabrication steps are complete. 


\section{CURRICULUM VITA}

NAME: Danming Wei

DOB: Shenyang, Liaoning, China - Jan 7, 1987

EDUCATION: $\quad$ B.S., Electrical Engineering

Dalian Jiaotong University, Dalian, China

2007-2011

M.S., Chemistry

Western Kentucky University, Kentucky, US

2013-2016

M.S., Electrical Engineering

University of Louisville, Kentucky, US

2016-2018

AWARDS:

The 2nd Place in Mobile Microrobot Challenge 2017

RESEARCH: Microrobot, Microsystem control, and Automation. 